\title{
ALGEBRAIC DEFORMATIONS OF POLARIZED VARIETIES
}

\author{
T. MATSUSAKA*)
}

Introduction. Let $V$ be a projectively embeddable complete non-singular variety of dimension $n>1$. Let $\boldsymbol{f}$ be a projective embedding of $V, U$ a nonsingular variety, $W$ a non-singular variety and $\varphi$ a morphism of $W$ onto $U$ such that $\varphi^{-1}\left(u_{0}\right)=\boldsymbol{f}(V)$ for some point $u_{0}$ of $U$. Denote by $\Sigma(V)$ the set of all those complete non-singular fibres $\varphi^{-1}(u), u \in U$, as we consider all possible $(\boldsymbol{f}, U, W)$. Suppose that we call members of $\Sigma(V)$ (algebraic) deformations of $V$ and propose to study $\Sigma(V)$ from the stand point of algebraic geometry, as a generalization of the case of curves. This has been taken up at least locally by Kodaira, Spencer, Kuranishi and others in the case of characteristic 0 from a little more general point of view of complex manifolds (cf. [9] and references given in [16]).

Within the frame work of algebraic geometry, one could ask if (a) there is a subset $\mathfrak{F}$ of $\Sigma(V)$ which is an algebraic family (of finite type, of course) of non-singular varieties in a projective space, containing every member of $\Sigma(V)$ up to an isomorphism. Or we could consider a suitable equivalence relation in $\Sigma(V)$ and consider $\left(\mathrm{a}^{\prime}\right)$ the problem (a) replacing "isomorphism" by "equivalence relation". But we shall consider here only (a) and not the problem $\left(a^{\prime}\right)$. When (a) is affirmative, to study $\Sigma(V)$ modulo the equivalence relation defined in terms of isomorphisms is reduced to study $\mathfrak{F}$ modulo the equivalence relation. Then we could ask if (b) the abstract quotient space $\mathfrak{T}$ of $\Sigma(V)$ modulo the equivalence relation has a structure of an algehraic variety. If (a) and (b) can be solved affirmatively, we would then propose to study (c) various properties of the algebraic variety $\mathfrak{T}$. The first major difficulty one encounters along this line of approach is that (a) is false even for well-known types of algebraic varieties. When $V$ is an Abelian variety, it is impossible to find $\mathfrak{F}$ in (a), and if one insists on finding something similar to $\mathfrak{F}$, then it would have to be an infinite union of irreducible algebraic families. Moreover, $\mathfrak{I}$ in this

Received December 21, 1966.

*) This work was done while the author was supported partially by N.S.F. 
case would have to be an infinite union of algebraic varieties, if the solution of (b) is possible at all. At least in the case of characteristic 0, this difficulty can be avoided in the case of Abelian varieties by introducing the concept of polarization (cf. [14], [25]) because of a theorem of Lefschetz which states that $3 X$ is ample if $X$ is a non-degenerate divisor. Therefore, we reformulate the definitions and problems accordingly in terms of polarized varieties. This still does not eliminate one more major difficulty in the case of characteristic $p$, since an example of a polarized Abelian variety $V$ shows that the self-intersection numbers of basic polar divisors, i.e. the ranks or the degrees, of members of $\Sigma(V)$ may not be bounded. This means that the solution of (a) and (b) are not possible within the frame work of algebraic varieties of finite types.

Therefore, we reformulate again the definitions and problems in terms of polarized varieties of ranks bounded by a positive integer $d$ and write $\Sigma(V, d)$ for $\Sigma(V)$. Then, when $n=2$, (a) has a solution, as we have shown essentially in [16] (cf. Theorem 3). When $n>2$, (a) is still an unsolved problem except for some special cases. In general, if (a) has a solution $\mathfrak{F}$, we shall call $\mathfrak{F}$ a universal family of $\Sigma(V, d)$. Let $\Sigma$ be a subset of $\Sigma(V, d)$ which admits a solution of (a) relative to $\Sigma$. We shall call it an admissible subset of $\Sigma(V, d)$ (a precise definition will be given later). Consider now the problem (b) with respect to $\Sigma$, using a universal family $\mathfrak{F}$. Very little is known about the solution of (b) in general, except for some special cases, notably the case of polarized Abelian varieties and the case of polarized Abelian varieties with complex multiplications (for references, see [17], in particular works of Siegel, Baily, Shimura and Mumford). In order to see if the solution of (b) is possible, we try to find a "good" universal family $\mathfrak{F}$ of $\Sigma$. Regarding members of $\mathfrak{F}$ as points on a Chow-variety or a Hilbert scheme, we get a locally closed algebraic variety $F$ of finite type from $\mathfrak{F}$. Then we regard $\mathfrak{F}$ as a "good" universal family, which we shall call a typical universal family, if, among other things, (i) the equivalence relation on $\Sigma$ induces on $F$ a closed equivalence relation, (ii) the orbits are equi-dimensional and the dimensions remain constant throughout $F$ and if (iii) special orbits are specializations of generic orbits over some field of references (a precise definition will be given later). We shall show (Theorems $5,6,7$ ) that such a good universal family exists for $\Sigma$ if we remove some ruled varieties from $\Sigma$. This removing process is technically not 
easy, but we can accomplish it by letting some good ruled varieties to remain. Then we shall show that (b) has a solution if we allow $\mathfrak{I}$ to be a $Q$-variety (cf. [15]) or a $Q$-space, which is a generalization of a locally closed algebraic set where the components are $Q$-varieties. Whether a solution of (b) is possible or not for $\Sigma$ within the frame work of algebraic varieties is an unsolved and, probably, a very difficult problem.

$\S 1$ is preliminary. In $\$ 2$, we redefine the concept of polarization, a slightly different one from [14], [25] and prove the existence of a basic polar divisor and the field of moduli. The latter is a useful concept as a first approximation to the moduli question, especially when (a) and (b) have no solutions. In $\$ 7$ we consider, as an example, the case when $V$ is a polarized variety of rank $d$, and is a generic complete intersection $U=H_{m_{1}} \cdots H_{m_{s}}$ of hypersurfaces of degrees $m_{i}$ in the projective space $\boldsymbol{P}^{N}$. First we show that a small deformation of $V$ (in the sense of algebraic deformation) is also a complete intersection of the similar kind as $V$ as long as its rank is $d$ (cf. Theorem 18.5 of [9] in the case of hypersurfaces). Then we consider the set $\Sigma$ of members of $\Sigma(V, d)$ which are of rank $d$ and are complete intersections of the same type as $V$. We show that $\Sigma$ is an admissible subset of $\Sigma(V, d)$ and that (b) has a solution in which $\mathfrak{T}$ is a $Q$-variety. Further we show that $\operatorname{dim} \mathfrak{T}=\sum h^{0}\left(U, \mathfrak{D}_{U}\left(m_{i}\right)\right)-s-\operatorname{dim} P G L(N)+\operatorname{dim} \operatorname{aut}(U)$, where $\mathfrak{5}_{U}$ is the sheaf of local rings on $U$ and aut $(U)$ the algebraic group of automorphisms of $U$ (cf. [14]; $U$ is considered as polarized). Moreover, we show that $\operatorname{dim}$ aut $(U)=0$ if $\sum m_{i}-N-1 \geqslant 0$ and, in the case of characteristic 0 , that $\mathfrak{I}$ is a $Q$-manifold which is similar to a non-singular variety (cf. [15]).

Notations, Conventions and Terminology. Basically we shall follow those of [23]. As to cohomological conventions and terminology, we shall follow [3], [20] and [26]. As to results on specializations, we shall follow [21]. For the convenience of the reader, we first list some of the basic notations which do not require detailed explanation.

$\mathfrak{C}_{l}(X)$ (resp. $\mathfrak{C}_{l}(X)^{+}$) The set of divisors (resp. positive divisors) which are linearly equivalent to $X$.

$\mathfrak{E}_{a}(X)$ (resp. $\mathfrak{E}_{a}(X)^{+}$) The set of divisors (resp. positive divisors) which are algebraically equivalent to $X$.

$\mathfrak{C}_{n}(X)$ (resp. $\left.\mathfrak{C}_{n}(X)^{+}\right)$The set of divisors (resp. positive divisors) which are numerically equivalent to $X$. 
$\mathbb{S}_{l}(V) \quad$ The group of divisors which are linearly equivalent to 0 on $V$.

$\mathscr{S S}_{a}(V) \quad$ The group of divisors which are algebraically equivalent to 0 on $V$.

$\mathscr{S}_{n}(V) \quad$ The group of divisors which are numerically equivalent to 0 on $V$.

$\mathscr{S}_{l}, \mathscr{S}_{a}, \mathscr{S}_{n} \quad$ will be used for the above when there is no danger of confusion.

$\mathfrak{t}(V) \quad$ The order of the torsion group $\mathscr{S}_{n} / \mathscr{S}_{a}$ of divisors of $V$.

$\Gamma_{g} \quad$ The graph of a rational map $g$.

$\Lambda(X) \quad$ The complete linear system determined by a divisor $X$.

$L(X) \quad$ The module of rational functions on a variety $V$, satisfying $\operatorname{div}(f)+X>0$ with respect to a $V$-divisor $X$.

$\sim \quad$ Linear equivalence of divisors.

$\mathfrak{Q}(X) \quad$ The (invertible) sheaf determined by a divisor $X$.

$\mathfrak{L}(V ; X) \quad$ The sheaf on $V$ determined by a $V$-divisor $X$.

$H^{i}(V, \mathfrak{Q}(X)) \quad$ The i-th cohomology group of $V$ with coefficients in $\mathfrak{Q}(X)$.

$H^{i}(V, X) \quad$ The same as above.

$h^{i}(V, \mathfrak{R}(X)) \quad$ The dimension of $H^{i}(V, \mathfrak{L}(X))$.

$h^{i}(V, X) \quad$ The same as above.

$l(X) \quad$ The dimension of $L(X),=h^{0}(V, \mathbb{R}(X))=\operatorname{dim} A(X)+1$.

$D_{U} \quad$ The sheaf of local rings on a variety $U$.

$\boldsymbol{P}^{N} \quad$ The projective space of dimension $N$.

$\chi(V, \mathfrak{Q}(X)) \quad$ The Euler-Poincaré characteristic of $\mathfrak{L}(X)$.

$\chi(V, X) \quad$ The same as above.

$\mathfrak{c}(Y) \quad$ The Chow-point of a positive cycle $Y$ in a projective space.

$C_{V} \quad$ A hyperplane section of a subvariety $V$ of a projective space.

$X \rightarrow X^{\prime}$ ref. $\mathfrak{D} . X^{\prime}$ is a specialization of $X^{\prime}$ over $\mathfrak{D}$.

Let $V$ be a complete variety, non-singular in codimension 1 , and $X$ a $V$-divisor. $L(X)$ is a finite dimensional vector space (cf. [23]). Let $f_{0}, \ldots, f_{r}$ be a basis of $L(X)$ and $\boldsymbol{f}$ the map of $V$ into a projective space determined by $x \rightarrow\left\langle f_{0}(x): \cdots: f_{r}(x)\right)$. When $\boldsymbol{f}$ is a projective embedding of $V$ and when $\Lambda(X)$ has no fixed point, we shall call $X$ an ample divisor on $V$ (very ample in the sense of Grothendieck); $\boldsymbol{f}$ will be called a non-degenerate projective 
embedding of $V$ determined by $X$ and will be denoted by $\boldsymbol{f}_{X} ; \Lambda(X)$ will be called an ample complete linear system. When a $V$-divisor $X$ has a property that a positive multiple of $X$ is ample, it will be called non-degenerate (ample in the sense of Grothendieck).

Suppose that a $V$-divisor $X$ satisfies the following conditions: (i) $X^{\prime}$ is ample whenever $X^{\prime} \in \mathfrak{C}_{a}(X)$; (ii) $h^{2}\left(V, \mathfrak{R}\left(m X^{\prime}\right)\right)=0$ for such $X^{\prime}$ whenever $i>0, m>0$. Then we shall call $X$ sufficiently ample.

Let $\mathfrak{U}$ be a set of $V$-divisors. Denote by $\mathfrak{P}(V, \mathfrak{u})$ the set of all possible $\boldsymbol{f}_{X}(V)$ for ample divisors $X$ in $\mathfrak{U}$. Denote by $\mathfrak{p}(V, \mathfrak{u})$ the set of Chow-points of members of $\mathfrak{P}(V, \mathfrak{u})$.

Suppose now that $V$ is non-singular in codimension $r$ and that it embedded in a projective space. By an algebraic family $\mathfrak{F}$ of positive $r$ cycles on $V$, we understand a set of positive cycles of dimension $r$ on $V$ such that the set of Chow-points of them forms a locally closed algebraic set $F$ in a projective space. When $F$ is absolutely irredusible (i.e. the point set attached to a variety), $\mathfrak{\mho}$ will be called an irreducible algebraic family; $F$ will be called the Chow-variety of $\mathfrak{F}$. Let, in general, $\mathfrak{F}_{i}$ be a subset of $\mathfrak{F}$ such that the set of Chow-points of members of it forms a component $F_{i}$ of $F$. Then $\mathfrak{F}_{i}$ will be called a component of $\mathfrak{F}$. When $F$ is normally algebraic over a field $k, \mathfrak{F}$ will be said to be normally algebraic over $k$. This is equivalent to say that $\mathfrak{F}$ is normally algebraic if $F$ is a $k$-closed set minus a $k$ closed set. When $F_{i}$ is defined over a field $k$, we shall say that $\mathfrak{F}_{i}$ is defined over $k$. Further notions on algebraic varieties can be carried over to the families $\mathfrak{F}_{i}, \mathfrak{F}$ by identifying cycles with their Chow-points which would not require detailed explanation.

Let $V$ be again a complete variety, non-singular in codimension 1 and $\mathfrak{A}$ an algebraic family of positive $V$-divisors. Let $X_{0}$ be an arbitrary but fixed member of $\mathfrak{A}$. When the class of $X-X_{0}$ with respect to linear equivalence exhaust the points of the Picard variety of $V$ by varying $X$ in $\mathfrak{A}$, we shall say that $\mathfrak{A}$ is a total family. When $l(X)$ is constant throughout $\mathfrak{A}$ and when $\Lambda(X) \subset \mathfrak{A}$ for every $X$ in $\mathfrak{A}$, we shall say that $\mathfrak{A}$ is a complete family. As one can see easily, using the concept of the Picard variety, a complete total family is an irreducible algebraic family (cf. [12]).

Known basic results which will be used quite often, but scattered in the literature, will be stated as Theorem A, B, C, ..., Proposition A, B, C, ...., Lemma A, B, C, ..., with references. 


\section{§1. Preliminaries.}

Theorem A. Let $V$ and $V^{\prime}$ be non-singular subvarieties of projective spaces, $X$ a $V$-divisor, $X^{\prime}$ a $V^{\prime}$-divisor and 5 a discrete valuation-ring such that $\left(V^{\prime}, X^{\prime}\right)$ is a specialization of $(V, X)$ over $\mathfrak{D}$. Then $\chi(V, X)=\chi\left(V^{\prime}, X^{\prime}\right)$. When $V=V^{\prime}$ and $X \equiv X^{\prime} \bmod \mathscr{S}_{a}$, then $\chi(V, X)=\chi\left(V^{\prime}, X^{\prime}\right)$.

This can be found in [7], [3].

Proposition 1. Let $V^{n}$ and $V^{\prime n}$ be subvarieties of projective spaces having no singular subvarieties of codimension $1, X$ a V-divisor, $X^{\prime} a V^{\prime}$-divisor and $5 a$ discrete valuation-ring such that $\left(V^{\prime}, X^{\prime}\right)$ is a specialization of $(V, X)$ over $\$$. Then we have the following results:

(a) When $X \sim 0$, then $X^{\prime} \sim 0$;

(b) When $X \equiv 0 \bmod \mathfrak{S S}_{a}$, then $X^{\prime} \equiv 0 \bmod \mathfrak{S}_{a}$;

(c) When $V$ and $V^{\prime}$ are non-singular and $X \equiv 0 \bmod \mathscr{S}_{n}$, then $X^{\prime} \equiv 0 \bmod \mathscr{S}_{n}$;

(d) When $V$ and $V^{\prime}$ are non-singular, $X^{(n)}=X^{\prime(n)}$, i.e., the self-intersection numbers are invariant by specializations.

Proof. [a] is contained in [21]. (b) follows from the definition of algebraic equivalence, fundamental properties of Chow-forms (cf. [2]) and from the extended Zariski connectedness theorem (cf. [1] and [4]). (c) follows from the definition of numerical equivalence, (b) and from the finiteness of $\mathfrak{t}(V)$ (cf. [13]). There is a positive integer $m$ such that $X+m C_{V}, m C_{V}$ (resp. $\left.X^{\prime}+m C_{V^{\prime}}, \quad m C_{V^{\prime}}\right)$ are ample and that their higher cohomology groups vanish. Then $l\left(X+m C_{V}\right)=l\left(X^{\prime}+m C_{V^{\prime}}\right)$ and $l\left(m C_{V}\right)=l\left(m C_{V^{\prime}}\right)$ by Theorem A. Denote by $G(*)$ the support of the Chow-variety of the complete linear system $\Lambda(*)$. Then $G\left(X^{\prime}+m C_{V^{\prime}}\right)$ is the uniquely determined specialization of $G\left(X+m C_{V}\right)$ over 5 over $(V, X) \rightarrow\left(V^{\prime}, X^{\prime}\right)$ ref. 5 . The same is true for $G\left(m C_{V}\right)$ and $G\left(m C_{V^{\prime}}\right)$. Hence, when $\left(x_{1}^{\prime}, \ldots, x_{u}^{\prime}\right),\left(y_{1}^{\prime}, \ldots, y_{v}^{\prime}\right)$ are sets of points in $G\left(m C_{V^{\prime}}\right), G\left(X^{\prime}+m C_{V^{\prime}}\right)$ respectively, there are sets $\left(x_{1}, \ldots, x_{u}\right)$, $\left(y_{1}, \ldots, y_{v}\right)$ in $G\left(m C_{V}\right), G\left(X+m C_{V}\right)$ such that $\left(V^{\prime}, X^{\prime},\left(x^{\prime}\right),\left(y^{\prime}\right)\right)$ is a specialization of $(V, X,(x),(y))$ over $\mathfrak{D}$. Expressing $X \sim\left(X+m C_{V}\right)-m C_{V}$, $X^{\prime} \sim\left(X^{\prime}+m C_{V^{\prime}}\right)-m C_{V^{\prime}}$ and combining the above remark with the compatibility of specializations with intersection-product, we get (d) easily.

Proposition 2. Let $V$ be a non-singular subvariety of a projective space and $X$ a sufficiently ample divisor on $V$. Then the set $\mathfrak{夭}_{a}(X)^{+}$is a complete total family of divisors. 
Proof. $l(X)$ is constant throughout $\mathfrak{E}_{a}(X)^{+}$by the definition and Theorem A. Let $X_{0}$ be a fixed member of $\mathfrak{E}_{a}(X)^{+}$and $Z$ an arbitrary divisor on $V$, algebraically equivalent to 0 . By the definition of a sufficiently ample divisor, $Z+X_{0}$ is sufficiently ample and there is a member $X$ of $\mathfrak{S}_{a}(X)^{+}$ such that $Z+X_{0}-X \sim 0$. It follows that the class of $X-X_{0}, X \in \mathfrak{c}_{a}(X)^{+}$, exhaust the points of the Picard variety of $V$. From these two facts and from (a) of Proposition 1, our proposition follows easily.

Proposition 3. Let $V$ and $V^{\prime}$ be non-singular subvarieties of projective spaces, $X$ a $V$-divisor, $X^{\prime}$ a $V^{\prime}$-divisor and 5 a discrete valuation-ring such that $\left(V^{\prime}, X^{\prime}\right)$ is a specialization of $(V, X)$ over 5 . Suppose that $X^{\prime}$ is sufficiently ample on $V^{\prime}$. Then $X$ is sufficiently ample on $V$ and $l(X)=l\left(X^{\prime}\right)=\chi(V, X)=\chi\left(V^{\prime}, X^{\prime}\right)$.

Proof. This follows at once from the definition of a sufficiently ample divisor, the upper-semi continuity (Proposition 2.3 of the Appendix) and from Theorem A.

Theorem B. Let $V$ and $V^{\prime}$ be non-singular subvarieties of projective spaces and 5 a discrete valuation-ring such that $V^{\prime}$ is a specialization of $V$ over $\subseteq$. Then the Picard variety of $V$ and that of $V^{\prime}$ have the same dimension.

This is a result due to Grothendieck (cf. [6], p. 14).

Proposition 4. Let $V$ and $V^{\prime}$ be subvarieties of projective spaces which are non-singular in codimension 1 . Let $X$ (resp. $X^{\prime}$ ) be a positive divisor on $V$ (resp. $\left.V^{\prime}\right)$, belonging to a complete total family $\mathfrak{A}$ (resp. $\left.\mathfrak{U}^{\prime}\right)$ of positive divisors on $V$ (resp. $\left.V^{\prime}\right)$ such that $l(X)=l\left(X^{\prime}\right)$. Let $\subseteq$ be a discrete valuation-ring such that $\left(V^{\prime}, X^{\prime}\right)$ is a specialization of $(V, X)$ over $\mathfrak{D}$. Then, when $A$ (resp. $\left.A^{\prime}\right)$ is the support of the Chow-variety of $\mathfrak{A}$ (resp. $\left.\mathfrak{A}^{\prime}\right), A^{\prime}$ is the uniquely determined specialization of $A$ over $\subseteq$ over the given specialization.

Proof. This proposition follows easily from the definitions, Theorem B and (a), (b) of proposition 1 .

Theorem C. Let $V$ be a complete non-singular variety. Then the group $\mathscr{S}_{n} / \mathscr{S}_{a}$ is finite. Let $V$ and $V^{\prime}$ be non-singular subvarieties of projective spaces, 5 a discrete valuation-ring such that $V^{\prime}$ is a specialization of $V$ over 5 and $p$ the characteristic of the residue field of $\mathfrak{S}$. Let $\left(\mathfrak{S}_{n}(V) / \mathfrak{S}_{a}(V)\right)_{q}\left(\operatorname{resp} .\left(\mathbb{S}_{n}\left(V^{\prime}\right) /\left(\mathfrak{S}_{a}\left(V^{\prime}\right)\right)_{q}\right)\right.$ be the q-primary part of the torsion group of divisors on $V$ (resp. $V^{\prime}$ ) for a prime $q \neq 0 \bmod p$. Then the specialization induces a canonical isomorphism between $\left(\mathscr{S}_{n}(V) / \mathscr{S}_{a}(V)\right)_{q}$ and $\left(\mathbb{S}_{n}\left(V^{\prime}\right) / \mathscr{S}_{a}\left(V^{\prime}\right)\right)_{q}$. 
Proof. The first part is contained in [13]. As to the second part, see [6], Remark. 3. 10.

Theorem D. Let $\leqq$ be a discrete valuation-ring with the quotient field $k$; let $V$ and $W$ be non-singular subvarieties of projective spaces and $T$ the graph of an isomorphism between $V$ and $W$ such that $V, W, T$ are defined over $k$. Let $X$ (resp. $Y$ ) be a non-degenerate divisor on $V$ (resp. W), rational over $k$, such that $Y=T(X)$. Let $\left(V^{\prime}, W^{\prime}, X^{\prime}, Y^{\prime}, T^{\prime}\right)$ be a specialization of $(V, W, X, Y, T)$ over $\mathfrak{D}$ and assume that $V^{\prime}, W^{\prime}$ are non-singular and that $X^{\prime}$ (resp. $\left.Y^{\prime}\right)$ is also non-degenerate on $V^{\prime}$ (resp. $\left.W^{\prime}\right)$. Then $T^{\prime}$ is the graph of an isomorphism between $V^{\prime}$ and $W^{\prime}$ if one of $V^{\prime}, W^{\prime}$ is not a ruled variety.

This is the Theorem 2 of [16].

Theorem $\mathrm{D}^{\prime}$. Using the same notations and assumptions as in Theorem $D$, except that $V^{\prime}, W^{\prime}$ may be both ruled varieties, assume that $T^{\prime}$ contains a component $T^{\prime \prime}$ whcih is a birational correspondence between $V^{\prime}$ and $W^{\prime}$. Then $T^{\prime}$ is the graph of an isomorphism between $V^{\prime}$ and $W^{\prime}$.

Proof. The proof of Theorem $D$ given in [16] is in fact a proof of this theorem. When $V^{\prime}$ or $W^{\prime}$ is not a ruled variety, $T^{\prime}$ contains a component $T^{\prime \prime}$ as described in the above theorem (cf. Theorem 1 of. [16], which is essentially due to Abhyankar). Then we established Theorem $D$ in (16) by proving our Theorem $D^{\prime}$.

\$2. Polarized varieties. Let $V$ be a complete non-singular variety. Let $\mathfrak{M}$ be a subset of the ring of integers and $\mathfrak{X}$ a set of $V$-divisors satisfying the following conditions:

$\left(P_{1}\right)$ There is a finite set of prime numbers $\left(p_{1}, \ldots, p_{r}\right)$ such that $\mathfrak{M}$ consists of 0 and the integers $\pm \Pi_{i} p_{i}^{e_{i}}$, where the $e_{i}$ are non-negative integers, $p_{i} \neq 1$ for all $i$ and at least one of the $e_{i}$ is positive;

$\left(P_{2}\right) \quad\left(p_{1}, \ldots, p_{r}\right)$ consists of the characteristic of the universal domains and the prime divisors of $\mathrm{t}(V)^{1)}$;

$\left(P_{3}\right) \quad \mathfrak{X}$ contains an ample divisor $X$;

1) When we deal with geometry involving specializations over discrete valuarings of unequal characteristics, then we shall be dealing with the characteristic 0 and the fixed characteristic $p$ in this paper. In such a case, even when $\mathrm{V}$ is defined over a field of characteristic 0 , we include $p$ in the set $\left(p_{1}, \ldots, p_{r}\right)$. Otherwise, we shall be dealing with varieties over fields of the fixed characteristic, and there shall be no ambiguity in this definition. 
$\left(P_{4}\right) \quad$ When $Y$ is a $V$-divisor, it is contained in $\mathfrak{X}$ if and only if there is a member $Z$ of $\mathfrak{X}$ and a pair $(r, s)$ of integers, not in $\mathfrak{M}$, such that $r Y \equiv s Z$ $\bmod \mathscr{S}_{a}$.

When there is a pair $(\mathfrak{M}, \mathfrak{X})$ satisfying $\left(P_{1}\right)-\left(P_{4}\right), \mathfrak{X}$ will be called a structure set of polarization of type $\mathfrak{M}$. When that is so, the pair $(V, \mathfrak{X})$ will be called a polarized variety of type $\mathfrak{M}$. A member of $\mathfrak{X}$ will be called a polar divisor. A class of $V$-divisors determined by a polar divisor is called a polar divisor class. A polarized variety $(V, \mathfrak{X})$ of type $\mathfrak{M}$ is said to be defined or rational over a field $k$ if $V$ is defined over $k$ and if there is a rational polar divisor over $k$. From now on, polarized varieties will be denoted by a bold faced letter. Automorphisms and isomorphisms of polarized varieties are defined as usual. A projective embedding $\boldsymbol{f}$ of a polarized variety $\boldsymbol{V}$ is a projective embedding of the underlying variety $V$ of $\boldsymbol{V}$, determined by an ample linear system containing a polar divisor. $\boldsymbol{f}(\boldsymbol{V})$ will then denote a polarized variety of the same type as $\boldsymbol{V}$, whose underlying variety is $\boldsymbol{f}(V)$ such that a hyperplane section of $\boldsymbol{f}(V)$ is a polar divisor. The definition of $\mathfrak{P}(V, \mathfrak{u}), \mathfrak{p}(V, \mathfrak{u})$ can be extended to the case of polarized varieties in the obvious manner, allowing only projective embeddings as polarized varieties in the definitions.

Remark 2.1. The definition of polarized varieties given here is somewhat different from the previous definition given in [14], [25]. The old definition is modified in this manner so that it is more suitable to algebraic varieties, other than Abelian varieties. It is possible to consider a subset $\mathfrak{M M}^{\prime}$ of the ring of integers, containing $\mathfrak{M}$ in $\left(P_{1}\right),\left(P_{2}\right)$, to define polarizations. But this will not add anything further. On the contrary, if $\mathfrak{M}^{\prime}$ is contained in $\mathfrak{M}$ and we consider the structure of polarization of type $\mathfrak{M C}^{\prime}$, slightly different phenomena will occur. But as long as $\mathfrak{M i}^{\prime}$ contains the characteristic of the universal domain, results will not be substantially different from those which will be developed in this paper.

Let $(V, \mathfrak{X})=\boldsymbol{V},\left(V^{\prime}, \mathfrak{X}^{\prime}\right)=\boldsymbol{V}^{\prime}$ be two polarized varieties of the same type. Let $k$ be a field of definition of $\boldsymbol{V}$. Assume that there is a discrete valuation-ring $\subseteq$ of $k$ with the following properties: (i) $V^{\prime}$ is a specialization of $V$ over 5 ; (ii) when $X$ is a $k$-rational polar divisor of $V$ and $X^{\prime}$ a specialization of $X$ over $\mathfrak{D}$, then $X^{\prime}$ is in $\mathfrak{X}^{\prime}$. Then we shall say that $V^{\prime}$ is a

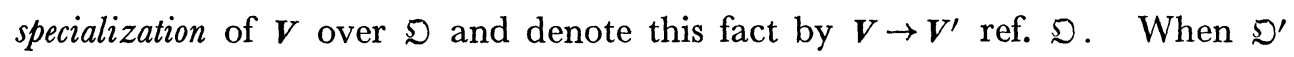


is a discrete valuation-ring which is dominated by $\mathfrak{D}$, we shall say also that $\boldsymbol{V}^{\prime}$ is a specialization of $\boldsymbol{V}$ over $\mathfrak{S}^{\prime}$.

The absolute values of the self-intersection numbers $X^{(n)}, n=\operatorname{dim} \boldsymbol{V}$, of polar divisors of $\boldsymbol{V}$ attain the smallest positive value $d . \quad d$ will be called the rank or the degree of the polarization. Let $X_{0}$ be a polar divisor of $\boldsymbol{V}$ and assume that it satisfies the following conditions: (i) A $V$-divisor $Y$ is in $\mathfrak{X}$ if and only if $Y \equiv r X_{0} \bmod \mathscr{S}_{a}$ with $r \notin \mathfrak{M}$, where $\mathfrak{M}$ is the type of $\boldsymbol{V}$; (ii) when $Z$ is an ample polar divisor, then $Z \equiv s X_{0} \bmod \leftrightarrow_{a}$ with a suitable positive integer $s$, not in $\mathfrak{M}$. When such a polar divisor $X_{0}$ exists, it is called a basic polar divisor. A basic polar divisor class of $\boldsymbol{V}$ with respect to algebraic equivalence is uniquely determined. Moreover, the rank is given by $X_{0}^{(n)}$.

Proposition 5. Let $\boldsymbol{V}$ be a polarized variety. Then there is a basic polar divisor.

Proof. Let \&s be the additive group of $V$-divisors, where $V$ is the underlying variety of $\boldsymbol{V}$. The group $\mathscr{S} / \mathscr{S}_{a}$ is finitely generated (cf. [19]). Therefore, it is possible to find a set $\left(D_{1}, \ldots, D_{u}, T_{1}, \ldots, T_{V}\right)$ of generators of $\mathscr{S S}^{5}$ modulo $\mathfrak{S}_{a}$ such that the $T_{j}$ are torsion divisors and that $\sum a_{i} D_{i}+\sum b_{j} T_{j} \equiv 0 \bmod \mathscr{S}_{a}$ if and only if $a_{i}=0$ and $b_{j} \equiv 0 \bmod t_{j}$, where the $t_{j}$ denote the order of $T_{j}$ modulo $\mathscr{S}_{a}$. Let $Y$ be a polar divisor and $Y \equiv \sum a_{i} D_{i}+\sum b_{j} T_{j} \bmod \mathscr{G}_{a} . \quad$ Let $e$ be the G.C.D. of the $a_{i}, b_{j}$ in $Z-\mathfrak{M}$, where $\mathfrak{M}$ is the type of polarization of $\boldsymbol{V}$, and set $\sum a_{i} D_{i}+\sum b_{j} T_{j}= \pm e Z$. The signature is so determined that a positive multiple of $Z$ is algebraically equivalent to an ample divisor. When that is so, the self-intersection number of $Z$ is positive. Let $X$ be an arbitrary polar divisor. There are two integers $r$ and $s$, not in $\mathfrak{M}$, such that $r X \equiv s Z \bmod \mathfrak{S}_{a}$. We may assume, without loss of generality, that $(r, s)=1$ since $r$ and $s$ are relatively prime to $\mathfrak{t}(V)$. Set $X \equiv \sum c_{i} D_{i}+\sum d_{j} T_{j} \bmod \mathscr{S}_{a}$ and $Z \equiv \sum a_{i}^{\prime} D_{i}+\sum b_{j}^{\prime} T_{j} \bmod \mathscr{S}_{a}$. Then we get $r c_{i}=s a_{i}^{\prime}$ and $r d_{j} \equiv s b_{j}^{\prime} \bmod t_{j}$. From the first we get $a_{i}^{\prime}=r a_{i}^{\prime \prime}$ where the $a_{i}^{\prime \prime}$ are also integers. The $t_{j}$ are divisors of $\mathrm{t}(V)$. Hence they are relatively prime to $s$. It follows that there is an integer $x$ such that $s x \equiv 1 \bmod t_{j}$. We then get $r x d_{j} \equiv b_{j}^{\prime} \bmod t_{j}$ from the second relation. Thus $Z \equiv r\left(\sum a_{i}^{\prime \prime} D_{i}+\sum x d_{j} T_{j}\right) \bmod \mathscr{E}_{a}$. Set $Z^{\prime}= \pm\left(\sum a_{i}^{\prime \prime} D_{i}+\sum x d_{j} T_{j}\right)$, where the signature is so chosen that a positive multiple of $Z^{\prime}$ is algebraically equivalent to an ample divisor. Clearly $Z^{\prime}$ is a polar divisor and the self- 
intersection number of $Z^{\prime}$ is strictly less than that of $Z$ when $r \neq \pm 1$. This process leads us to find a basic polar divisor.

Let $\boldsymbol{V}$ be a polarized variety and $k_{0}$ a fixed field. Consider the set $S$ of fields $K$, containing $k_{0}$, having the following properties: (i) When $\boldsymbol{W}$ is isomorphic to $\boldsymbol{V}$, a generic specialization of $\boldsymbol{W}$ over $K$ is still isomorphic to $\boldsymbol{V}$; (ii) There is a polarized variety $\boldsymbol{U}$, isomorphic to $\boldsymbol{V}$, defined over a separably generated extension of $K$. When $S$ is not empty and contains the smallest field $K_{0}, K_{0}$ will be called the field of moduli of $\boldsymbol{V}$ over $k_{0}$. The existence of the field of moduli of $\boldsymbol{V}$ over $k_{0}$ can be proved almost exactly the same way as in [14] with a few trivial modification of the proofs. The definition of the field of moduli in [14] is different in appearance from the present one which is due to Shimura [22]. But they are clearly the same, which will become clear also in the following context. One different aspect of our present situation than that of [14] is that we can establish the existence of the field of moduli always, whereas we needed an assumption (valid for characteristic 0 or for curves and Abelian varieties in general) to establish it in [14]. Because of this and because of the fact that we shall need some of the results of [14], we shall reestablish the existence of the field of moduli here.

We proved in [12] that when $V$ is a complete non-singular variety and $Z$ an ample divisor on it, there is a positive integer $m_{0}$ such that $\mathfrak{c}_{a}(m Z)^{+}$ is a complete total family for $m>m_{0}$. However we shall need later a more precise result than this. Therefore we shall begin proving it.

Theorem 1. Let $V^{n}$ be a complete non-singular variety, $C$ an ample divisor and $X$ an arbitrary divisor on $V$. Set $\chi(V, m C)=g(m), \chi(V, X+m C)=g_{x}(m)$. Then (a) there is a constant $c_{1}$, depending only on $g(x), g_{X}(x)$ and the intersectionnumbers $I\left(X^{(j)} \cdot C^{(n-j)}\right)$ such that every divisor $Z$ in $\mathfrak{S}_{n}(X+t C)$ is ample and that $h^{i}(V, Z)=0$ for $i>0$ if $t>c_{1}$; (b) Let $r X \equiv C \bmod \mathbb{S}_{n}$ for some positive integer $r$, then there is a constant $c_{2}$, depending only on $g(x)$ and $r$, such that every divisor in $\mathfrak{c}_{n}(t X)$ is sufficiently ample for $t>c_{2}$.

Proof. There is a constant $c_{1}^{\prime}$, depending only on $g(x), g_{X}(x)$ and the intersection-numbers $I\left(X^{(j)} . C^{(n-j)}\right)$, such that $h^{i}(V, X+t C)=0$ when $i>0$, $t>c_{1}^{\prime}$ by Proposition 3.5 of the Appendix. Let $Z$ be an arbitrary member of $\mathfrak{E}_{n}(X+t C)$. Then $Z-t C=X^{\prime} \equiv X \bmod \mathscr{S}_{n}$ and $\chi(V, X+m C)=\chi\left(V, X^{\prime}+m C\right)$ by Proposition 3.2 of the Appendix. Moreover, various intersection numbers 
of $X$ with $C$ and those of $X^{\prime}$ and $C$ coincide. Therefore, whenever $t>c_{1}^{\prime}$, every $Z$ in $\mathfrak{E}_{n}(X+t C)$ satisfies $h^{i}(V, Z)=0$ for $i>0$ and $h^{0}(V, Z)=\chi(V, Z)=$ $\chi(V, X+t C)=h^{0}(V, X+t C)$. Since $g_{X}(x)$ is a polynomial by Proposition 3.1 of the Appendix, there is a constant $c_{1}^{\prime \prime}$, depending only on $g_{X}(x)$, such that $g_{X}(t)>1$ when $t>c_{1}^{\prime \prime}$. This is because $g_{X}(x)>1$ when $x$ is sufficiently large. Set $\max \left(c_{1}^{\prime}, c_{1}^{\prime \prime}\right)=c_{1}^{*}-1$. Set $d_{0}=I\left\{C^{(n-1)} \cdot\left(X+c_{1}^{*} C\right)\right\} \cdot\left(C^{(n)}-2\right)+C^{(n)}$. When $Z$ is an arbitrary member of $\varsigma_{n}\left(X+c_{1}^{*} C\right), Z+d C$ is ample for $d>d_{0}$ (cf. [23], Chap. IX, Corollary of Theorem 13). Set $c_{1}=c_{1}^{*}+d_{0}$. This $c_{1}$ satisfies the requirements of (a).

Suppose now that $r X \equiv C \bmod \dot{S}_{n}$. When $s$ is a non-negative integer, we have $\chi(V, s X+m C)=\chi\left(V, s^{\prime} X+(m+q) C\right)$ if $s=s^{\prime}+q \cdot r$ and $0 \leqslant s^{\prime}<r$ (cf. Proposition 3.2 of the Appendix). Set $\chi(V, s X+m C)=g_{s X}(m)$ for $0 \leqslant s<r$. Set also $\chi(V, m X)=h(m)$. We have $g_{s X}(m)=h(s+m r)$ and $h(r m)=g(m)$ by Proposition 3.2 of the Appendix. $h(m)$ is a polynomial in $m$ when $m$ is positive by Lemma 3.1 of the Appendix. It follows that $g_{s X}(x)$ depends only on $g(x)$ and $s$. The same is true for various intersection-numbers of $s X$ with $C$, since these are given by the $(s / r)^{j} \cdot C^{(n)}$. Therefor, for each $s$ such that $0 \leqslant s<r$, there is a constant $c_{1, s}$, depending only on $g(x)$ and $s$, such that every divisor $Z$ in $\mathfrak{c}_{n}(s X+m C)$ is ample and satisfies $h^{i}(V, Z)=0$ for $i>0$ if $m>c_{1, s}$. Let $c_{2}^{\prime}$ be the maximum of the $c_{1, i}$. Then every such $Z$ is ample and satisfies $h^{i}(V, Z)=0$ for $i>0$ if $m>c_{2}^{\prime}$. Set $c_{2}=$ $c_{2}^{\prime} \cdot(r+1)$. Every divisor in $\mathfrak{C}_{n}(t X)$ has then these two properties too when $t>c_{2}$. This proves $(b)$.

Theorem E. Let $V$ be a complete non-singular variety and $\mathfrak{A}$ a complete total family of positive divisors on $V$, consisting of ample divisors. Then $\mathfrak{B}(V, \mathfrak{X})$ has a structure of an algebraic variety. When $\mathfrak{A}$ is defined over a field $k$ of definition of $V, \mathfrak{p}(V, \mathfrak{A})$ is defined over $k$. Moreover, $\mathfrak{p}(V, \mathfrak{X})$ has the smallest field of definition and this field is also the smallest field of definition of its closure.

This is Theorem 3 of [14].

Theorem F. Let $\boldsymbol{V}$ be a polarized variety and aut $(\boldsymbol{V})$ the set of automorphisms of $\boldsymbol{V}$. Then aut $(\boldsymbol{V})$ is an algebraic group.

This is Theorem 6 of [14].

As an immediate corollary to Theorems $E$ and $F$, we get the following result:

Corollary. Let $\boldsymbol{V}$ be a polarized variety, $q$ the dimension of the Picard 
variety of $\boldsymbol{V}$ and $\mathfrak{A}$ a complete total family of positive polar divisors of $\boldsymbol{V}$, consisting of ample polar divisors. Let $X$ be a member of $\mathfrak{A}$ and $l(X)=N+1$. Then $\operatorname{dim} \mathfrak{p}(\boldsymbol{V}, \mathfrak{X})=q+\operatorname{dim} P G L(N)-\operatorname{dim} \operatorname{aut}(\boldsymbol{V})$.

Theorem G. Let $V$ be a non-singular subvariety of a projective space and $\mathfrak{A}$ a complete total family of divisors on $V$. Let $k$ be a field of definition of $V$ over which $\mathfrak{A}$ has a rational member. Then $\mathfrak{A}$ is defined over $k$.

This is [11]-II, Proposition 1.

Lemma A. Let $\boldsymbol{V}$ be a polarized variety and $Z$ a basic polar divisor of $\boldsymbol{V}$. Let $m$ and $r$ be two positive integers such that $\mathfrak{\Xi}_{a}(m Z)^{+}, \mathbb{E}_{a}(r Z)^{+}$are complete total families, consisting of ample polar divisors. When $\mathfrak{p}\left(\boldsymbol{V}, \mathfrak{E}_{a}(m Z)\right)$ is defined over a field $K$, then $\mathfrak{p}\left(\boldsymbol{V}, \mathfrak{c}_{a}(r Z)\right)$ is defined over an algebraic extension of $K$.

Lemma B. Let the notations and assumptions be as in Lemma $A$. When $\mathfrak{p}\left(\boldsymbol{V}, \mathfrak{\subseteq}_{a}(m Z)\right)$ is defined over a field $K, \mathfrak{p}\left(\boldsymbol{V}, \mathfrak{\complement}_{a}(\operatorname{smZ})\right)$ is defined over $K$ whenever $s$ is a positive integer such that $\mathfrak{C}_{a}(\operatorname{sm} Z)^{+}$is also a complete total family consisting of ample polar divisors.

Lemma C. Let $U$ be a non-singular subvariety of a projective space, $X$ a positive $U$-divisor and $m$ a positive integer which is prime to the characteristic $p$. Assume that $\mathfrak{夭}_{a}(X)^{+}, \mathfrak{}_{a}(m X)^{+}$are both irreducible algebraic families. Let $A, A_{m}$ be respectively the Chow-varieties of them. Then, when $k$ is a common field of definition for $U$ and $A_{m}, A$ is defined over a separably algebraic extension of $k$.

These Lemmas A, B, C are respectively Lemmas 8, 9, 10 of [14]. As a consequence of Lemma $\mathrm{C}$, we get the following result.

Lemma 1. Let $V$ be a non-singular subvariety of a projective space and $X a$ non-degenerate $V$-divisor. Let $r$ be a positive integer, $\neq 0(p)$, and assume that $\mathfrak{E}_{a}(X)^{+}, \mathfrak{E}_{a}(r X)^{+}$are both complete total families of divisors, consisting of ample divisors. When $k$ is a field of definition of $\mathfrak{p}\left(V, \mathfrak{\mho}_{a}(r X)\right), \mathfrak{p}\left(V, \mathfrak{\mho}_{a}(X)\right)$ is defined over a separably algebraic extension of $k$.

Proof. Since $\mathfrak{p}\left(V, \mathfrak{c}_{a}(r X)\right)$ is defined over $k$, it has a rational point $w$ over a separable algebraic extension $K$ of $k$. Let $W$ be the variety corresponding to $w$ and $\boldsymbol{f}$ an isomorphism between $V$ and $W$ such that $\boldsymbol{f}(\boldsymbol{r} X) \equiv C_{W} \bmod \mathbb{S}_{a}$. By our assumptions, $\mathfrak{E}_{a}(\boldsymbol{f}(X))^{+}, \quad \mathfrak{E}_{a}(\boldsymbol{r} \boldsymbol{f}(X))^{+}$are complete total families, consisting of ample divisors. Moreover, the latter contains a rational divisor over $K$. Hence it is defined over $K$ by Theorem $G$. When 
that is so, the former family is defined over a separable algebraic extension of $K$ by Lemma $C$. Our lemma follows then from Theorem $\mathrm{E}$.

Theorem 2. Let $\boldsymbol{V}$ be a polarized variety of type $\mathfrak{M}$ and $k_{0}$ a fixed field. Then the field of moduli of $\boldsymbol{V}$ over $k_{0}$ exists. When $Z$ is a basic polar divisor of $\boldsymbol{V}$ and $m$ a positive integer, not in $\mathfrak{M}$, such that $\mathfrak{c}_{a}(m Z)^{+}$is a complete total family of ample polar divisors, the smallest field $K$ of definition of $\mathfrak{p}\left(\boldsymbol{V}, \mathfrak{c}_{a}(m Z)\right)$ over $k_{0}$ is the field of moduli of $\boldsymbol{V}$ over $k_{0}$.

Proof. Denote by $\mathfrak{R}$ the set of positive integers $s$, not contained in $\mathfrak{M}$ such that $\mathfrak{E}_{a}(s Z)^{+}$is a complete total family of ample polar divisors. By Theorem 1 , a positive integer $s$, not contained in $\mathfrak{M}$, is contained in $\mathfrak{R}$ whenever it is sufficiently large. Therefore, when $r$ is a large integer not in $\mathfrak{M}$, then $r m$ is also in $\mathfrak{R}$. Let $r$ be such a large positive integer. Denote by $k, k_{r}$ respectively the smallest fields of definitions of $\mathfrak{p}\left(\boldsymbol{V}, \mathfrak{C}_{a}(m Z)\right)$, $\mathfrak{p}\left(\boldsymbol{V}, \mathfrak{c}_{a}(r m Z)\right)$ over $k_{0}$. By Lemma $\mathrm{B}$ and Lemma $1, k_{r}$ is contained in $k$ and $k$ is a separable algebraic extension of $k_{r}$.

We contend that $k=k_{r}$. For this, it is enough to show that $\mathfrak{p}\left(\boldsymbol{V}, \mathfrak{c}_{a}(m Z)\right)$ has no other conjugate than itself over $k_{r}$. Let $\boldsymbol{V}^{\prime}$ be an element of $\mathfrak{P}\left(\boldsymbol{V}, \mathfrak{C}_{a}(r m Z)\right)$. Let $\boldsymbol{f}$ be an isomorphism between $\boldsymbol{V}$ and $\boldsymbol{V}^{\prime}$ and $Z^{\prime}=\boldsymbol{f}(Z)$. Clearly $\mathfrak{P}\left(\boldsymbol{V}, \mathfrak{E}_{a}(r m Z)\right)=\mathfrak{P}\left(\boldsymbol{V}^{\prime}, \mathfrak{E}_{a}\left(\boldsymbol{C}_{\boldsymbol{V}^{\prime}}\right)\right)=\mathfrak{P}\left(\boldsymbol{V}^{\prime}, \mathfrak{E}_{a}\left(r m Z^{\prime}\right)\right)$. Let $\alpha$ be an automorphism of $\bar{k}_{r}$ over $k_{r}$ and $K$ a field of rationality of $\boldsymbol{V}^{\prime}, Z^{\prime}, C_{\boldsymbol{V}^{\prime}}$ over $k_{r}$. Extend $\alpha$ to an isomorphism of $K$ into the universal domain and denote it by the same letter $\alpha$. We have $\mathfrak{p}\left(\boldsymbol{V}, \mathfrak{c}_{a}(r m Z)\right)^{\alpha}=\mathfrak{p}\left(\boldsymbol{V}, \mathfrak{\subseteq}_{a}(r m Z)\right)$ and $\mathfrak{p}\left(\boldsymbol{V}^{\prime}, \mathfrak{E}_{a}\left(m Z^{\prime}\right)\right)^{\alpha}=\mathfrak{p}\left(\boldsymbol{V}^{\prime \alpha}, \mathfrak{E}_{a}\left(m Z^{\prime \alpha}\right)\right)$. From the first equality we see that $\boldsymbol{V}^{\prime \alpha}$ is in $\mathfrak{p}\left(\boldsymbol{V}, \mathfrak{C}_{a}(r m Z)\right)$ and, when that is so, there is an isomorphism $\boldsymbol{g}$ between $\boldsymbol{V}^{\prime}$ and $\boldsymbol{V}^{\prime \alpha}$. This shows that $\mathfrak{P}\left(\boldsymbol{V}^{\prime}, \mathfrak{c}_{a}\left(m Z^{\prime}\right)\right)=\mathfrak{P}\left(\boldsymbol{V}^{\prime \alpha}, \mathfrak{c}_{a}\left(m \boldsymbol{g}\left(Z^{\prime}\right)\right)\right)$. On the other hand, $r m Z^{\prime} \equiv C_{V^{\prime}} \bmod \quad \mathscr{S}_{a}$ and this implies $r m Z^{\prime \alpha} \equiv C_{V^{\prime \alpha}} \bmod \quad \mathscr{S}_{a}$. Since $\boldsymbol{g}$ is an isomorphism of polarized varieties and since hyperplane sections of $\boldsymbol{V}^{\prime}, \boldsymbol{V}^{\prime \alpha}$ are polar divisors, it follows that $r m \boldsymbol{g}\left(Z^{\prime}\right) \equiv \boldsymbol{g}\left(r m Z^{\prime}\right) \equiv \boldsymbol{g}\left(C_{\boldsymbol{V}^{\prime}}\right) \equiv$ $C_{V^{\prime \alpha}} \bmod \mathscr{S}_{a}$. Thus, we have $m Z^{\prime \alpha} \equiv m \boldsymbol{g}\left(Z^{\prime}\right) \bmod \mathfrak{S}_{a}$ since $r$ is not in $\mathfrak{M}$. Consequently, $\mathfrak{B}\left(\boldsymbol{V}^{\prime}, \mathfrak{C}_{a}\left(m Z^{\prime}\right)\right)=\mathfrak{P}\left(\boldsymbol{V}^{\prime \alpha}, \mathfrak{E}_{a}\left(m Z^{\prime \alpha}\right)\right)$. When we combine this with the second equality we have established, we see that $\mathfrak{p}\left(\boldsymbol{V}, \mathfrak{c}_{a}(m Z)\right)$ has no conjugate than itself over $k_{r}$.

Since $\mathfrak{p}\left(\boldsymbol{V}, \mathfrak{E}_{a}(m Z)\right)$ is defined over $k, k$ belongs to $S$, the set of fields which enter in the definition of the field of moduli of $\boldsymbol{V}$ over $k_{0}$. We contend that every field in $S$ contains $k$. Let $L$ be an element of $S$. There is a 
polarized variety $V^{\prime \prime}$ defined over a separably generated extension $L^{\prime}$ of $L$ and isomorphic to $\boldsymbol{V}$. Let $X^{\prime \prime}$ be a rational polar divisor of $\boldsymbol{V}^{\prime \prime}$ over $L^{\prime}$ and $u$ a positive integer such that $u m$ is also in $N$. um $X^{\prime \prime}$ is rational over $L^{\prime} . \quad \widetilde{C}_{a}\left(u m X^{\prime \prime}\right)^{+}$is a complete total family of ample polar divisors and contains a rational divisor over $L^{\prime}$. Hence this family is defined over $L^{\prime}$ by Theorem $G$ and $\mathfrak{p}\left(\boldsymbol{V}^{\prime \prime}, \mathfrak{E}_{a}\left(u m X^{\prime \prime}\right)\right)$ is defined over $L^{\prime}$ by Theorem $E$. $\mathfrak{B}\left(\boldsymbol{V}^{\prime \prime}, \mathfrak{c}_{a}\left(u m X^{\prime \prime}\right)\right)=\mathfrak{P}\left(\boldsymbol{V}, \mathfrak{c}_{a}(u m t Z)\right)$ for some positive integer $t$, not contained in $\mathfrak{M}$. Therefore, $L^{\prime}$ contains $k_{u t}$ and $k_{u t}=k$ as we have proved. Let $\alpha$ be an isomorphism of $L^{\prime}$ into the universal domain over $L$. By the definition of $L, \boldsymbol{V}^{\prime \prime \alpha}$ is isomorphic to $\boldsymbol{V}$. Replacing $\boldsymbol{V}^{\prime \prime}, L^{\prime}$ by $\boldsymbol{V}^{\prime \prime \alpha}, L^{\prime \alpha}$, we see that $L^{\prime \alpha}$ contains $k$ by the above result. Since this is so for all possible $\alpha$ and since $L^{\prime}$ is separably generated over $L$, it follows that $L^{\prime}$ contains $k$. Our theorem is thereby proved.

Corollary. Let $m$ be a positive integer such that $m Z$ is a sufficiently ample polar divisor of $\boldsymbol{V}$. Then, when $K^{\prime}$ is the smallest field of definition of $\mathfrak{p}\left(\boldsymbol{V}, \mathfrak{\subseteq}_{a}(m Z)\right)$, $k_{0} \cdot K^{\prime}$ is the field of moduli of $\boldsymbol{V}$ over $k_{0}$.

$\S 3$. Polarized varieties (continued). A main purpose of this paragraph is to show that specializations of polarized varieties can be realized in projective spaces up to isomorphisms. In order to do this, we shall begin with a few lemmas.

Lemma 2. Let $V^{n}$ be a complete abstract variety, non-singular in codimension 1 and $\Lambda$ an arbitrary linear system of positive dimension on $V$. Let $k$ be a common field of definition of $V$ and $A$ and $\left(x_{1}, \ldots, x_{r}\right)$ a set of independent generic points of $V$ over $k$. Then there is a member $X$ of $\Lambda$ which goes through the points $x_{i}$ if and only if $\operatorname{dim} \Lambda \geq r$. If such $X$ exists and the set of such $X$ forms a finite set, $X$ is the only element of the set, $\operatorname{dim} \Lambda=r$ and $X$ is rational over $k\left(x_{1}, \ldots, x_{r}\right)$.

This is well known and easy to prove.

Lemma 3. Let $V^{n}\left(r e s p . V^{\prime n}\right)$ be a complete abstract variety, nonsingular in codimension 1 and $X$ (resp. $\left.X^{\prime}\right)$ a $V$-(resp. $V^{\prime}$-) divisor. Let $k$ be a common field of rationality of $V$ and $X, \subseteq$ a discrete valuation-ring of $k$ and assume that $(V, X) \rightarrow$ $\left(V^{\prime}, X^{\prime}\right)$ ref. 5 and that $l(X)=l\left(X^{\prime}\right)$. Let $k^{\prime}$ be the residue field of $\leqq$ and $X_{1}^{\prime}, \ldots, X_{m}^{\prime}$ $m$ independent generic members of $\Lambda\left(X^{\prime}\right)$ over $k^{\prime}$. Then there is a set of $m$ independent generic members $X_{1}, \ldots, X_{m}$ of $\Lambda(X)$ over $k$ and a discrete valuation-ring $\mathfrak{D}^{*}$ of a common field $K$ of rationality of the $X_{i}$ over $k$ such that $\mathfrak{D}^{*}$ dominates $\mathfrak{D}$ and that $\left(V^{\prime}, X^{\prime}, X_{1}^{\prime}, \ldots, X_{m}^{\prime}\right)$ is a specialization of $\left(V, X, X_{1}, \ldots, X_{m}\right)$ over $5^{*}$. 
Proof. Set $\operatorname{dim} \Lambda(X)=\operatorname{dim} \Lambda\left(X^{\prime}\right)=r . \quad$ Let the $x_{\alpha i}(1 \leqslant i \leqslant r ; 1 \leqslant \alpha \leqslant m)$ be independent generic points of $V$ over $k$. By Lemma 2, there is a uniquely determined divisor $X_{\alpha}$ in $\Lambda(X)$, for each $\alpha$, such that $X_{\alpha}$ goes through the $x_{\alpha i}$ for all $i$; moreover, $X_{\alpha}$ is rational over the field $k(x)$. Denote by $V_{(s)}$ the product of $V$ by itself s-times. Then there is a positive divisor $W$ on $V_{(m r)} \times V_{(m)}$, rational over $k$, such that $W \cdot\left((x) \times V_{(m)}\right)=(x) \times X_{1} \times \cdots \times X_{m}$ (cf. [23], Chap. VIII, Theorem 6).

By Lemma 2, there are $m r$ independent generic points $x_{\alpha i}^{\prime}$ of $V^{\prime}$ over $k^{\prime}$ such that $X_{\alpha}^{\prime}$ goes through $x_{\alpha i}^{\prime}$ for $1 \leqslant i \leqslant r$. When that is so, $\left(x^{\prime}\right)$ is a specialization of $(x)$ over $D$, over the specialization $(V, X) \rightarrow\left(V^{\prime}, X^{\prime}\right)$ ref. $D$. There is a discrete valuation-ring $\mathfrak{N}^{*}$ of $k(x)$, dominating $\mathfrak{N}$, such that $(V, X,(x)) \rightarrow\left(V^{\prime}, X^{\prime},\left(x^{\prime}\right)\right)$ ref. $\mathfrak{D}^{*}$. Let $\left(V_{(m r)}, V_{(m)}, W\right) \rightarrow\left(V^{\prime}{ }_{(m r}, V^{\prime}{ }_{(m)}, W^{\prime}\right)$ ref. $\mathfrak{D}^{*}$ be an extension of the above specialization. Since $W$ is rational over $k$ and since $\mathfrak{D}^{*}$ dominates $\mathfrak{D}$, it follows that $W^{\prime}$ is rational over $\overline{k^{\prime}}$. Then $W^{\prime}\left(x^{\prime}\right)$ is the uniquely determined specialization of $W(x)$ over $\mathfrak{D}^{*}$ over these specializations, since specializations and intersection-product are compatible. $W^{\prime}\left(x^{\prime}\right)$ is of the form $X_{1}^{\prime \prime} \times \cdots \times X_{m}^{\prime \prime}$ where the $X_{\alpha}^{\prime \prime}$ are $V^{\prime}$-divisors. Since linear equivalence is compatible with specializations, $X_{\alpha}^{\prime \prime}$ is a member of $\Lambda\left(X^{\prime}\right)$. Since $X_{\alpha}$ goes through the $x_{\alpha i}$ for all $i, X_{\alpha}^{\prime \prime}$ goes through the $x_{\alpha i}^{\prime}$ for all $i$. Consequently, $X_{\alpha}^{\prime}=X_{\alpha}^{\prime \prime}$ by Lemma 2. Our lemma is thereby proved.

Lemma 4. Let $V, V^{\prime}, X, X^{\prime}, \mathfrak{D}^{*}, K$, the $X_{\alpha}$ and the $X_{\alpha}^{\prime}$ be as in Lemma 3 and $K^{\prime}$ the residue field of $\mathfrak{D}^{*}$. Let the $g_{\alpha}^{\prime}$ be functions on $V^{\prime}$, defined over $K^{\prime}$, such that div $\left(g_{\alpha}^{\prime}\right)=X_{\alpha}^{\prime}-X_{1}^{\prime}\left(\right.$ set $\left.g_{1}^{\prime}=1\right)$. Then there are functions $g_{\alpha}$ on $V$, defined over $K$, such that div $\left(g_{\alpha}\right)=X_{\alpha}-X_{1}, g_{1}=1$, and that $\left(V^{\prime}, X^{\prime},\left(X_{\alpha}^{\prime}\right)_{1 \leqslant \alpha \leqslant m},\left(g_{\alpha}^{\prime}\right)_{1 \leqslant \alpha \leqslant m}\right)$ is a specialization of $\left(V, X,\left(X_{\alpha}\right)_{1 \leqslant \alpha \leqslant m},\left(g_{\alpha}\right)_{1 \leqslant \alpha \leqslant m}\right)$ over $5^{*}$.

Proof. Let $P$ be a generic point of $V$ over $K$ and $P^{\prime}$ a generic point of $V^{\prime}$ over $K^{\prime}$ such that $P^{\prime}$ is a specialization of $P$ over $\mathfrak{D}^{*}$. When $\mathfrak{D}^{\prime}$ is the specialization-ring of the specialization, it is integrally closed since $V^{\prime}$ is irreducible; it is then a discrete valuation-ring since $\operatorname{dim}_{K}(P)=\operatorname{dim}_{K^{\prime}}\left(P^{\prime}\right)$ (cf. [21], Proposition 5, Theorem 15). Moreover, when $\mathfrak{S}^{*}$ is the maximal ideal of $\mathfrak{D}^{*}, \mathfrak{P}^{*} \mathfrak{S}^{\prime}$ is the maximal ideal of $\mathfrak{N}^{\prime}$ (cf. [21], Corollary 2 of Theorem 15). Let the $h_{i}$ be the functions on $V$, defined over $K$, such that $h_{1}=$ $1, \operatorname{div}\left(h_{i}\right)=X_{i}-X_{1}$. Let $t$ be a generator of $\mathfrak{B}^{*}$. Since $t$ generates the valuation-ideal of $\mathfrak{D}^{\prime}$, there is an integer $e_{i}$ such that $t^{e_{i}} h_{i}(P)$ is a unit in $\mathfrak{D}^{\prime}$. Setting them as $h_{i}^{\prime}(P)$, we see the existence of the functions $g_{i}^{\prime \prime}$ on $V^{\prime}$, 
defined over $K^{\prime}$, such that $h_{i}^{\prime}(P) \rightarrow g_{i}^{\prime \prime}\left(P^{\prime}\right)$ ref. $\mathfrak{D}^{\prime}$. By the definition, the $g_{i}^{\prime \prime}$ are specializations of the $h_{i}^{\prime}$ over $\coprod^{\prime}$ and $\operatorname{div}\left(g_{i}^{\prime \prime}\right)=X_{i}^{\prime}-X_{1}^{\prime}$ (cf. [21], Theorem 20). Hence, there are constants $c_{i}^{\prime}$ in $K^{\prime}$ such that $g_{i}^{\prime}=c_{i}^{\prime} g_{i}^{\prime \prime}$. Let the $c_{i}$ be elements of $\mathfrak{D}^{*}$ such that $c_{i} \rightarrow c_{i}^{\prime}$ ref. $\mathfrak{D}^{*}$. When we set $g_{i}=c_{i} h_{i}^{\prime}$, the $g_{i}$ satisfy our requirements.

Proposition 6. Let $V^{n}$ (resp. $\left.V^{\prime n}\right)$ be a complete non-singular abstract variety, $X\left(\right.$ resp. $\left.X^{\prime}\right)$ an ample divisor on $V\left(r e s p . V^{\prime}\right)$ and assume that $l(X)=l\left(X^{\prime}\right)$. Let $k$ be a common field of rationality of $V$ and $X, \subseteq$ a discrete valuation-ring of $k$ and assume that $\left(V^{\prime}, X^{\prime}\right)$ is a specialization of $(V, X)$ over 5 . Let $\tilde{\boldsymbol{f}}^{\prime}$ be a non-degenerate projective embedding $\boldsymbol{f}_{X}$, of $V^{\prime}$. Then, there is a field $K$ which contains $k$, a discrete valuation-ring $\mathfrak{D}^{\prime}$ of $K$ which dominates 5 and a non-degenerate projective embedding $\boldsymbol{f}=\boldsymbol{f}_{X}$ of $V$ such that $\left(V^{\prime}, X^{\prime}, \Gamma_{\tilde{\boldsymbol{f}}^{\prime}}\right)$ is a specialization of $\left(V, X, \Gamma_{\boldsymbol{f}}\right)$ over $\mathfrak{D}^{\prime}$.

Proof. Let $k^{\prime}$ be the residue field of $\mathfrak{D}$. Since $X^{\prime}$ is rational over $k^{\prime}$, $\Lambda\left(X^{\prime}\right)$ is defined over $k^{\prime}$. Set $l(X)=l\left(X^{\prime}\right)=N$ and let the $X_{\alpha}^{\prime}$ be $N+1$ independent generic members of $\Lambda\left(X^{\prime}\right)$ over $k^{\prime}$. Let the $g_{\alpha}^{\prime}$ be the functions on $V^{\prime}$, defined over $k^{\prime}$, such that $\operatorname{div}\left(g_{\alpha}^{\prime}\right)=X_{\alpha}^{\prime}-X_{0}^{\prime} \quad$ (we set $g_{0}^{\prime}=1$ ). We apply Lemmas 3 and 4 to our situation. Then there is a field $K$ which

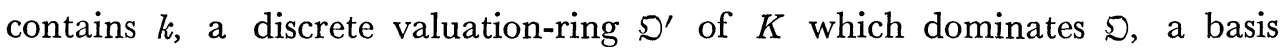
$g_{0}, \ldots, g_{N}$ of the module $L(X)$ over $K$ such that $\left(V^{\prime}, X^{\prime},\left(g^{\prime}\right)\right)$ is a specialization of $(V, X,(g))$ over $\mathfrak{S}^{\prime}$. $(g)$ (resp. $\left(g^{\prime}\right)$ ) determines a projective embedding $\boldsymbol{f}\left(\operatorname{resp} . \boldsymbol{f}^{\prime}\right)$ of $V\left(\operatorname{resp} . V^{\prime}\right)$, Let $\Gamma\left(\operatorname{resp} . \Gamma^{\prime}\right)$ be the graph of $\boldsymbol{f}\left(\operatorname{resp} . \boldsymbol{f}^{\prime}\right)$ and $T$ a specialization of $\Gamma$ over $\mathfrak{S}^{\prime}$. We contend that $T=\Gamma^{\prime}+T^{\prime}$ where $p r_{1} T^{\prime}=0, p r_{2} T^{\prime}=0$. In fact, $\operatorname{dim} T=\operatorname{dim} \Gamma^{\prime}$ and, from the definition of specializations of functions, it easily follows that $\Gamma^{\prime}$ is contained in the support of $T$. Hence $\Gamma^{\prime}$ is a component of $T$. The rest of the assertion follows from the compatibility of specializations with algebraic projection.

Next we contend that $T^{\prime}=0$. A divisor on $V$ is in $\Lambda(X)$ if and only if it is of the form $\boldsymbol{f}^{-1}(H)$ for a suitable hyperplane $H$ and the same is true for $V^{\prime}, \boldsymbol{f}^{\prime}$ and $\Lambda\left(X^{\prime}\right)$. Let $K^{\prime}$ be the residue field of $\mathfrak{D}^{\prime}$ and $H_{1}, \ldots, H_{r}$ (resp. $H_{1}^{\prime}, \ldots, H_{r}^{\prime}$ ) independent generic hyperplanes over $K$ (resp. $K^{\prime}$ ). Then $\left(H^{\prime}\right)$ is a specialization of $(H)$ over $\mathfrak{J}^{\prime}$ and the same is true for $p r_{1}\left(T \cdot\left(V^{\prime} \times H_{1}^{\prime} \cdots H_{r}^{\prime}\right)\right)$ and $\boldsymbol{f}^{-1}\left(H_{1} \cdots H_{r}\right)$ by the compatibilities of specializations with intersection-product and algebraic projection. If $T^{\prime}$ has a component which projects to a variety of dimension $r>0$, it would follow that $\operatorname{pr}_{1}\left(T \cdot\left(V^{\prime} \times H_{1}^{\prime} \cdots H_{r}^{\prime}\right)\right)-{f^{\prime-1}}^{-1}\left(H_{1}^{\prime} \cdots H_{r}^{\prime}\right) \supsetneqq 0$. The first term is a speciali- 
zation over $D^{\prime}$ of the intersection-product of $r$ properly intersecting members of $\Lambda(X)$. The second term is the intersection-product of $r$ properly intersecting members of $\Lambda\left(X^{\prime}\right)$. Then the strict inequality leads to a contradiction since specializations are compatible with specializations. If a component of $T^{\prime}$ projects to a point on the second factor, it must be of the form $V^{\prime} \times$ (a point) which is against to the fact that $p r_{1} T^{\prime}=0$. Hence we have proved our assertion.

Thus we have shown that there is a non-degenerate projective embedding $\boldsymbol{f}\left(\right.$ resp. $\left.\boldsymbol{f}^{\prime}\right)$ of $V\left(\right.$ resp. $\left.V^{\prime}\right)$ determined by $X$ (resp. $X^{\prime}$ ) such that $\boldsymbol{f}$ is defined over $K$ and that $\left(V, X, \Gamma_{\boldsymbol{f}}\right) \rightarrow\left(V^{\prime}, X^{\prime}, \Gamma_{\boldsymbol{f}^{\prime}}\right)$ ref. $\mathfrak{S}^{\prime}$. There is a projective transformation $\boldsymbol{h}^{\prime}$ (of the ambient space of $\boldsymbol{f}^{\prime}\left(V^{\prime}\right)$ ) such that $\tilde{\boldsymbol{f}}^{\prime}=\boldsymbol{h}^{\prime} \circ \boldsymbol{f}^{\prime} . \quad \boldsymbol{h}^{\prime}$ is uniquely determined by a defining matrix $\left(c_{i j}^{\prime}\right)$ and we can find a matrix $\left(c_{i j}\right)$, with the $c_{i j}$ in some extension of $K$, such that $\left(c_{i j}^{\prime}\right)$ is a specialization of $\left(c_{i j}\right)$ over $\mathfrak{D}^{\prime}$. Let $\boldsymbol{h}$ be the projective transformation (of the ambient space of $\boldsymbol{f}(V))$ determined by the matrix $\left(c_{i j}\right)$. Then it is easy to see that $\Gamma_{\boldsymbol{h}^{\prime}}$ is a specialization of $\Gamma_{\boldsymbol{h}}$ over $\mathfrak{S}^{\prime}$. When that is so, $\left(V^{\prime}, X^{\prime}, \Gamma_{\boldsymbol{h}^{\prime}} \circ \boldsymbol{f}^{\prime}\right)$ is a specialization of $\left(V, X, \Gamma_{\boldsymbol{h}} \circ \boldsymbol{f}\right)$ over $5^{\prime}$ by the compatibilities of specializations with intersection-product and algebraic projection (cf. [23], Propositions 10 and 11 of Chapter VIII too). Our proposition is thereby proved.

Proposition 6 allows us to change embeddings in specializations. To supplement this we shall have the following proposition.

Proposition 7. Let $V, V^{\prime}, X, X^{\prime}, k, 5$ be as in Proposition 6. Let $\boldsymbol{f}$ be a non-degenerate projective embedding of $V$ determined by $X$. Then there is a projective transformation $\boldsymbol{h}$ in the ambient space of $\boldsymbol{f}(V)$ and a non-degenerate projective embedding $\boldsymbol{f}^{\prime}$ of $V^{\prime}$ determined by $X^{\prime}$ such that $\left(V^{\prime}, X^{\prime}, \Gamma_{\boldsymbol{f}^{\prime}}\right)$ is a specialization of $(V, X$, $\left.\Gamma_{\boldsymbol{h}} \circ f\right)$ over 5 .

Proof. Since $X^{\prime}$ is ample, there is a non-degenerate projective embed$\operatorname{ding} \boldsymbol{f}^{\prime}$ of $V^{\prime}$ determined by $X^{\prime}$. By Proposition 6, there is a non-degenerate projective embedding $\boldsymbol{f}^{*}$ of $V$, determined by $X$, having the property described in that proposition. $f$ and $f^{*}$ differ by a projective transformation. Hence we have our proposition.

Before we pass to the next paragraph, we shall add one definition. Let $\boldsymbol{V}$ be a polarized variety, $Z$ a basic polar divisor and $V$ the underlying variety of $\boldsymbol{V} . \quad \chi(V, m Z)$ is a polynomial in $m$ by Proposition 3.1 of the Appendix. We shall denote this polynomial also by $\chi(\boldsymbol{V}, m \boldsymbol{Z})$. It is indepen- 
dent of the choice of $Z$ by Proposition 3.2 of the Appendix and is uniquely determined by $\boldsymbol{V}$. We shall call this polynomial the Hilbert polynomial or Hilbert function of $\boldsymbol{V}$.

$\S 4$. Deformations and universal families. Let $\boldsymbol{V}$ be a polarized variety. A polarized variety $\boldsymbol{W}$ will be called a deformation of $\boldsymbol{V}$ if the following conditions are satisfied. There is a finite set $\left(\boldsymbol{V}_{0}, \ldots, \boldsymbol{V}_{t}\right)$ of polarized varieties such that (a) $\boldsymbol{V}_{0}=\boldsymbol{V}, \boldsymbol{V}_{t}=\boldsymbol{W}$, that (b) either $\boldsymbol{V}_{i}$ is isomorphic to $\boldsymbol{V}_{i-1}$ or $\boldsymbol{V}_{i}$ (resp. $\boldsymbol{V}_{i-1}$ ) is a specialization of $\boldsymbol{V}_{i-1}$ (resp. $\boldsymbol{V}_{i}$ ) over some discrete valuation-ring. We shall denote the set of deformations of $\boldsymbol{V}$ by $\Sigma(\boldsymbol{V})$.

Remark 4.1. Our definition, in the case of characteristic 0, is narrower than that of Kodaira-Spencer (cf. [9]) in the following two points. We are restricting all the fibres which enter into their definition to be algebraic. Also we are considering algebraic varieties with extra structure, the structure of polarization.

Remark 4.2. When the characteristic is 0 and all the discrete valuationrings contain the field of rational numbers, $\Sigma(\boldsymbol{V})$ has the property that every member of it has one and the same rank as polarized varieties. This follows from the well-known theorem of Lefschetz on a criterion of a topological cycle to be an algebraic divisor (cf. [8], Chap. IV). On the other hand, when the universal domain is of characteristic $p$, a specialization of a polarized variety sometimes change ranks which was illustrated to the author by Nishi in the seminar at Brandeis, 1962. His example illustrates moreover that, when $\boldsymbol{V}$ is a suitably polarized Abelian variety in the universal domain of characteristic $p>0$, the set of equivalence classes of $\Sigma(\boldsymbol{V})$ defined in terms of isomorphisms of members of $\Sigma(\boldsymbol{V})$ is not a finite union of algebraic varieties ${ }^{2}$.

Because of the above remark, we shall consider the following subset of $\sum(\boldsymbol{V})$. Let $d$ be a positive integer such that $d \geqslant \operatorname{rank}(\boldsymbol{V})$. We shall denote by $\Sigma(\boldsymbol{V}, d)$ the subset of $\Sigma(\boldsymbol{V})$, consisting of those polarized varieties whose ranks are at most $d$. We shall also denote by $\Sigma_{0}(\boldsymbol{V}, d)$ the subset of $\Sigma(\boldsymbol{V}, d)$, consisting of polarized varieties of rank $d$. When the universal domain is of characteristic 0 and when the deformations are always over the field of

2) The author understands that Nishi intends to publish his example elsewhere. 
rational numbers, then $\Sigma(\boldsymbol{V})=\Sigma(\boldsymbol{V}, d)=\Sigma_{0}(\boldsymbol{V}, d)$ by the quoted theorem of Lefschetz.

From now on, we shall assume that the underlying varieties of polarized varieties we shall consider are non-singular subvarieties of projective spaces. By doing this we shall loose no generality by virtue of Proposition 6 .

Before we proceed to introduce some other fundamental concepts, we shall discuss briefly about deformations and Hilbert polynomials.

Lemma 5. Let $\boldsymbol{V}_{1}, \ldots, \boldsymbol{V}_{t}$ be polarized varieties such that either $\boldsymbol{V}_{i-1}$ and $V_{i}$ are isomorphic or $\boldsymbol{V}_{i}$ (resp. $\left.\boldsymbol{V}_{i-1}\right)$ is a specialization of $\boldsymbol{V}_{i-1}$ (resp. $\boldsymbol{V}_{i}$ ) over some discrete valuation-ring. Let the $Z_{i}$ be basic polar divisors of the $\boldsymbol{V}_{i}$ and $n$ the common dimension of the $\boldsymbol{V}_{i}$. Then there are positive integers $s_{1}, s_{t}$ such that $s_{1}{ }^{n} Z_{1}^{(n)}=s_{t}{ }^{n} Z_{t}^{(n)}$ and that $\chi\left(\boldsymbol{V}_{1}, m s_{1} Z_{1}\right)=\chi\left(\boldsymbol{V}_{t}, m s_{t} Z_{t}\right)$ for all integers $m$. If $\operatorname{rank}\left(\boldsymbol{V}_{1}\right)=\operatorname{rank}\left(\boldsymbol{V}_{t}\right)$, then $s_{1}=s_{t}$.

Proof. $\quad \boldsymbol{V}_{i-1}$ and $\boldsymbol{V}_{i}$ are related by an isomorphism or by a specialization and algebraic equivalence is preserved by either one. Therefore, such an operation induces a map $\mathfrak{夭}_{a}\left(m Z_{i-1}\right) \rightarrow \mathfrak{\Im}_{a}\left(m r_{i} Z_{i}\right)$ or $\mathfrak{夭}_{a}\left(m Z_{i}\right) \rightarrow \mathfrak{c}_{a}\left(m r_{i-1} Z_{i-1}\right)$ of algebraic equivalence classes, where $m$ is an integer and $r_{i-1}, r_{i}$ are some positive integers which are independent of $m$. Since the Euler-Poincaré characteristic relative to divisors is invariant by an isomorphism or by a specialization (cf. Theorem $A$ ), it follows that $\chi\left(\boldsymbol{V}_{i-1}, m Z_{i-1}\right)=\chi\left(\boldsymbol{V}_{i}, m r_{i} Z_{i}\right)$ and $\quad Z_{i-1}^{(n)}=r_{i}{ }^{n} Z_{i}^{(n)}$ or $\quad \chi\left(\boldsymbol{V}_{i-1}, m r_{i-1} Z_{i-1}\right)=\chi\left(\boldsymbol{V}_{i}, m Z_{i}\right) \quad$ and $Z_{i}^{(n)}=r_{i-1}{ }^{n} Z_{i-1}{ }^{(n)}$. From these and from Lemma 3.2 of the Appendix, our lemma follows easily.

Proposition 8. Let $\boldsymbol{V}$ be a polarized variety of rank $d$ and $\boldsymbol{W}$ a member of $\Sigma_{0}(\boldsymbol{V}, d)$. Then $\boldsymbol{V}$ and $\boldsymbol{W}$ have the same rank and the same Hilbert polynomial.

Proof. This follows at once from the definition and from Lemma 5.

We shall assume, from now on, that the discrete valuation-rings which enter in the definition of deformations contain one and the same basic field $k_{0} . k_{0}$ will be referred to as the basic field of deformations. Every field we shall consider will be assumed to contain $k_{0}$.

We shall introduce two more subspaces of $\Sigma(\boldsymbol{V})$. Let $\Sigma^{\prime \prime}(\boldsymbol{V}, d)$ be the set of polarized varieties $\boldsymbol{V}^{\prime}$ of ranks at most $d$, satisfying the following condition: There is a finite set of polarized varieties $\left(\boldsymbol{V}_{0}, \ldots, \boldsymbol{V}_{t}\right)$ such that $\boldsymbol{V}_{0}=\boldsymbol{V}^{\prime}, \quad \boldsymbol{V}_{t}=\boldsymbol{V}$ and that either $\boldsymbol{V}_{i}$ is a specialization of $\boldsymbol{V}_{i-1}$ over some 
discrete valuation-ring or $\boldsymbol{V}_{i}$ and $\boldsymbol{V}_{i-1}$ are isomorphic. Let $\Sigma^{\prime}(\boldsymbol{V}, d)$ be the set of polarized varieties $\boldsymbol{V}^{\prime}$ of ranks at most $d$, satisfying the following condition: There are two sets $\left(\boldsymbol{V}_{0}, \ldots, \boldsymbol{V}_{m}\right),\left(\boldsymbol{U}_{m+1}, \ldots, \boldsymbol{U}_{t}\right)$ of polarized varieties such that $\boldsymbol{V}_{0}=\boldsymbol{V}, \boldsymbol{U}_{t}=\boldsymbol{V}^{\prime}, \boldsymbol{V}_{m}=\boldsymbol{U}_{m+1}$ and that either $\boldsymbol{V}_{i-1}$ (resp. $\boldsymbol{U}_{j}$ ) is a specialization of $\boldsymbol{V}_{\boldsymbol{i}}$ (resp. $\boldsymbol{U}_{j-1}$ ) over some discrete valuation-ring or they are isomorphic. We further set $\Sigma^{\prime}(\boldsymbol{V}, d) \cap \Sigma_{0}(\boldsymbol{V}, d)=\Sigma_{0}^{\prime}(\boldsymbol{V}, d)$, $\Sigma^{\prime \prime}(\boldsymbol{V}, d)=\Sigma^{\prime \prime}(\boldsymbol{V}, d) \cap \Sigma_{0}(\boldsymbol{V}, d)$. Clearly, we have the following inclusion relations:

$$
\begin{aligned}
& \Sigma^{\prime \prime}(\boldsymbol{V}, d) \subset \Sigma^{\prime}(\boldsymbol{V}, d) \subset \Sigma(\boldsymbol{V}, d) \subset \Sigma(\boldsymbol{V}) \\
& \Sigma_{0}^{\prime \prime}(\boldsymbol{V}, d) \subset \Sigma_{0}^{\prime}(\boldsymbol{V}, d) \subset \Sigma_{0}(\boldsymbol{V}, d) .
\end{aligned}
$$

Sometimes, we shall call $\Sigma^{\prime}(\boldsymbol{V}, d)$ and $\Sigma_{0}^{\prime}(\boldsymbol{V}, d)$ local spaces of deformations at $\boldsymbol{V}$ and $\Sigma^{\prime \prime}(\boldsymbol{V}, d), \Sigma_{0}^{\prime \prime}(\boldsymbol{V}, d)$ quasi-local spaces of deformations.

In order to introduce the concept of a universal family, we shall consider one more definition. Let $\mathfrak{F}$ be an algebraic family of non-singular subvarieties of a projective space. Let $\mathfrak{M}$ be a multiplicatively closed set of integers such that every member of $\mathfrak{F}$ is the underlying variety of a polarized variety of type $\mathfrak{M}$ where hyperplane sections are polar divisors. For a given $\mathfrak{F}$, there is always such $\mathfrak{M}$ by virtue of Theorem $\mathrm{C}$. When we identify members of $\mathfrak{F}$ with such polarized varieties of type $\mathfrak{M}$, we shall denote the resulting set by $(\mathfrak{W}, \mathfrak{M})$ and call it an algebraic family of polarized varieties of type $\mathfrak{M}$. When there is no danger of confusion, we sometimes denote $(\mathfrak{F}, \mathfrak{M})$ simply by $\mathfrak{F}$.

Let $\boldsymbol{V}$ be a polarized variety of type $\mathfrak{M}$ and of rank at most $d$. Let $(\mathfrak{F}, \mathfrak{M})$ be an algebraic family of polarized varieties of type $\mathfrak{M}$ and $\Sigma$ a subset of $\Sigma(\boldsymbol{V}, d)$. Assume that $(\mathfrak{F}, \mathfrak{M})$ satisfies the following conditions:

$\left(U_{1}\right)$ Every member of $\Sigma$ is isomorphic to a member of $(\mathfrak{F}, \mathfrak{M})$;

$\left(U_{2}\right)$ When $k$ is a common field of definition of the components of $\mathfrak{F}$, a generic member of each component of $\mathfrak{F}$ over $k$ is a member of $\Sigma$. Then we shall call $(\mathfrak{F}, \mathfrak{M})$ a universal family of $\Sigma$. When $\Sigma$ is a subset of $\Sigma_{0}(\boldsymbol{V}, d),(\mathfrak{F}, \mathfrak{M})$ will be called a universal family of $\Sigma$ if it satisfies $\left(U_{1}\right)$, $\left(U_{2}\right)$ and $\left(U_{3}\right)$ Every member of $(\mathfrak{F}, \mathfrak{M})$ has the rank $d$.

Sometimes, when $(\mathfrak{F}, \mathfrak{M})$ is a universal family, we shall say that $\mathfrak{F}$ is a universal family if there is no danger of confusion. In this case, the identification of members of $\mathfrak{F}$ with members of $(\mathfrak{F}, \mathfrak{M})$ are assumed to have been done already. 
Remark 4.3. In the case of $\Sigma(\boldsymbol{V}, d)$, the above definition can be stated in the following equivalent form:

$\left(U_{1}^{\prime}\right)$ Every member of $\Sigma(\boldsymbol{V}, d)$ is isomorphic to a member of $(\mathfrak{F}, \mathfrak{M})$;

$\left(U_{2}^{\prime}\right)$ Every member of $(\mathfrak{F}, \mathfrak{M})$ is a member of $\Sigma(\boldsymbol{V}, d)$.

The same is true for $\Sigma^{\prime}(\boldsymbol{V}, d)$.

Unfortunately, the existence of a universal family for $\Sigma(\boldsymbol{V}, d), \Sigma_{0}(\boldsymbol{V}, d)$, $\Sigma^{\prime}(\boldsymbol{V}, d), \quad \Sigma_{0}^{\prime}(\boldsymbol{V}, d)$ is not known in general. But there are some important cases where the existence of a universal family can be established and we shall comment on this briefly here. First, clearly $\Sigma(\boldsymbol{V}, d)$ is a finite union of the $\Sigma_{0}\left(\boldsymbol{V}, d_{i}\right)$ with $d \geqslant d_{i}$. The same is true for $\Sigma^{\prime}(\boldsymbol{V}, d)$. Therefore, it is enough to discuss the problem for $\Sigma_{0}(\boldsymbol{V}, d), \Sigma_{0}^{\prime}(\boldsymbol{V}, d)$. Moreover, we may assume without loss of generality that $\boldsymbol{V}$ is of rank exactly $d$. By Proposition 8, members of $\Sigma_{0}(\boldsymbol{V}, d)$ have one and the same Hilbert polynomial. When that is so, the existence of a universal family of $\Sigma_{0}(\boldsymbol{V}, d)$ and that of $\Sigma_{0}^{\prime}(\boldsymbol{V}, d)$ follows from Proposition 4.3 of the Appendix if the following conjectural statement is true.

(*) Let $V$ be a non-singular subvariety of a projective space and $Z$ a non-degenerate divisor on $V$. Set $\chi(V, m Z)=g(m)$. Then there is a constant $c$, depending only on the polynomial $g(x)$ such that $m Z$ is ample for $m>c$.

When $\operatorname{dim} V=1, \quad(*)$ follows easily from the Theorem of RiemannRoch. In fact, $g(x)$ determines the genus $g$ uniquely, and when that is so, it is enough to take $c$ to be $2 g+1$.

When $\operatorname{dim} V=2$, (*) has been verified in [16].

When $V$ is an Abelian variety, $3 Z$ is always ample whenever $Z$ is a non-degenerate divisor on $V$ (a theorem of Lefschetz, generalized to the arbitrary characteristic case by Nishi, cf. [17]).

TheOREM 3. Let $\boldsymbol{V}$ be a polarized variety of dimension $n$ and a positive integer such that rank $(\boldsymbol{V}) \leqslant d$. If $(*)$ is true for dimension $n$, a universal family of $\Sigma_{0}(\boldsymbol{V}, d)$ and hence of $\Sigma(\boldsymbol{V}, d)$ exists.

If $\Sigma(\boldsymbol{V}, d)$ has a universal family, it is clear that all the other spaces of deformations admit universal families (cf. Proposition 4.3 of the Appendix). On the other hand, we can establish the following theorem without much difficulty.

Theorem 4. $\quad \Sigma^{\prime \prime}(\boldsymbol{V}, d)$ and $\Sigma_{0}^{\prime \prime}(\boldsymbol{V}, d)$ always admit universal families. 
Proof. Let $d_{0}=\operatorname{rank}(\boldsymbol{V}) . d \geqslant d_{0}$ by our definition. In general, when a polarized variety $\boldsymbol{U}$ is specialized to a polarized variety $\boldsymbol{U}^{\prime}$ over some discrete valuation-ring, a basic polar divisor is specialized to a polar divisor. Therefore, rank $(\boldsymbol{U})$ is always a multiple of rank $\left(\boldsymbol{U}^{\prime}\right)$, which shows that the rank of a member of $\Sigma^{\prime \prime}(\boldsymbol{V}, d)$ is bounded by $d$ and is a multiple of $d_{0}$. Set $e=\Pi_{i} d_{i}$, where the $d_{i}$ are positive multiples of $d_{0}$ but are bounded by $d$. Then, for any positive integer $r$, re has a property that the rank of a member of $\Sigma^{\prime \prime}(\boldsymbol{V}, d)$ divides $r e$.

When $X$ is a basic polar divisor of $\boldsymbol{V}$, there is a positive integer $r$ such that reX is sufficiently ample by Theorem 1 . Let $\boldsymbol{W}=\boldsymbol{V}_{0}, \ldots, \boldsymbol{V}_{t-1}$, $\boldsymbol{V}_{t}=\boldsymbol{V}$ be a finite set of polarized varieties such that $\boldsymbol{W}$ is in $\Sigma^{\prime \prime}(\boldsymbol{V}, d)$ and that either $\boldsymbol{V}_{i}$ is a specialization of $\boldsymbol{V}_{i-1}$ over some discrete valuationring or they are isomorphic. Then $\operatorname{rank}\left(\boldsymbol{V}_{i}\right)$ divides $r e$ and $\operatorname{rank}\left(\boldsymbol{V}_{i}\right)$ divides rank $\left(\boldsymbol{V}_{i-1}\right)$. Since algebraic equivalence is preserved by isomorphisms and specializations, we can find a polar divisor $Y_{i}$ on each $\boldsymbol{V}_{i}$ such that $Y_{t} \equiv m X$ $\bmod \mathscr{S}_{a}$, that either the specialization $\boldsymbol{V}_{i-1} \rightarrow \boldsymbol{V}_{i}$ extends to a specialization $Y_{i-1} \rightarrow Y_{i}$ or an isomorphism between $\boldsymbol{V}_{i-1}$ and $\boldsymbol{V}_{i}$ transforms $Y_{i-1}$ to $Y_{\imath}$ and that $Y_{0}$ is a basic polar divisor of $\boldsymbol{V}_{0}=\boldsymbol{W}$. Then $m^{n} d_{0}$ divides $r e$ when $n=$ $\operatorname{dim} \boldsymbol{V}$ and every $Y_{i}$ is sufficiently ample on $\boldsymbol{V}_{i}$ by Proposition 3.2 of the Appendix. Hence $\boldsymbol{W}$ has a non-degenerate projective embedding determined by $Y_{0}$, which maps $\boldsymbol{W}$ into the projective space of dimension $l(m X)-1$ with the resulting non-singular variety of degree $m^{n} d_{0}$ (cf. Proposition 3). Fix $r$ now once for all. Since $m^{n} d_{0}$ divides $r e$, the possibilities of $m$ is finite. Our theorem now follows easily from the fundamental results on Chow-forms (cf. [2]) and from Proposition 4.3 of the Appendix.

Corollary. Let $\mathfrak{F}$ be a universal family of $\Sigma^{\prime \prime}\langle\boldsymbol{V}, d)$. Consider the equivalence relation on $\mathfrak{F}$ in terms of isomorphisms of members of $\mathfrak{F}$ as polarized varieties. Let $F$ be the set of Chow-points of members of $\mathfrak{F}$ and $R$ the equivalence relation defined on $F$ by that of $\mathfrak{F}$. Then there is a locally closed set $W$ in a projective space, an open subset $F^{\prime}$ of $F$ and a continuous map $\beta$ of $F^{\prime}$ on $W$ such that the following properties hold: (a) $\beta$ induces on each component of $F^{\prime}$ a morphism; (b) $W$ is the quotient space of $F^{\prime}$ with respect to $R$ and $\beta$ the canonical map in the sense of topological space; (c) When $k$ is a common field of definition of the components of $F$ and $W$ and when $u$ is a point of $F^{\prime}$, corresponding to a member $\boldsymbol{U}$ of $\mathfrak{F}, k(\beta(u))$ is the field of moduli of $\boldsymbol{U}$ over $k$; (d) When $u$ is a generic point of a component of $F$ over $k, u$ is contained in $F^{\prime}$. When $F^{\prime}$ is given, $\beta$ and $W$ are uniquely 
determined up to a homeomorphism which induces a one-to-one birational correspondence on each component of $W$.

Proof. It is not hard to prove this corollary from Theorem 1, Theorem 2, Theorem $E$ and Theorem $G$, using the usual technique of eliminating undesirable points. Therefore we leave a proof for an exercise of the reader.

From the above corollary, it follows that the number of components $c(\boldsymbol{V}, d)$ of $\boldsymbol{W}$ and the dimensions of the components of $\boldsymbol{W}$ are uniquely determined by $\Sigma^{\prime \prime}(\boldsymbol{V}, d)$. $c(\boldsymbol{V}, d)$ could be called the number of local moduli components at $\boldsymbol{V}$. When $c(\boldsymbol{V}, d)=1$, we shall denote by $n(\boldsymbol{V}, d)$ the dimension of $W$ and call it the number of local moduli at $\boldsymbol{V}$, which is at most the number $m(\boldsymbol{V})$ in the case of characteristic 0 defined in (9).

Let $\boldsymbol{V}$ be a polarized variety of rank at most $d$ and $\Sigma$ a subset of $\Sigma(\boldsymbol{V}, d)$ (resp. $\left.\Sigma_{0}(\boldsymbol{V}, d)\right)$. An algebraic family $\mathfrak{F}$ of polarized varieties will be called a smooth universal family of $\Sigma$ if the following hold:

$\left(S U_{1}\right) \quad \mathfrak{F}$ is a universal family of $\Sigma$;

$\left(S U_{2}\right)$ When $\boldsymbol{U}$ is a member of $\mathfrak{F}, C_{U}$ is sufficiently ample;

$\left(S U_{3}\right)$ When $\boldsymbol{U}$ is a member of $\mathfrak{F}, \mathfrak{P}\left(\boldsymbol{U}, \mathfrak{c}_{a}\left(C_{U}\right)\right)$ is contained in $\mathfrak{F}$;

$\left(S U_{4}\right)$ No member of $\mathfrak{F}$ is contained in a hyperplane and the set of hyperplane sections forms a complete linear system on each member of $\mathfrak{F}$; $\left(S U_{5}\right) \quad C_{U}^{(n)}$ is a constant, independent of a member $\boldsymbol{U}$ of $F$ if $\operatorname{dim} \boldsymbol{V}=n$.

We shall say that $\Sigma$ is an admissible subset of $\Sigma(\boldsymbol{V}, d)\left(\operatorname{resp} . \Sigma_{0}(\boldsymbol{V}, d)\right)$ if: (a) There is a universal family of $\Sigma$, contained in $\Sigma$, and a member of $\Sigma(\boldsymbol{V}, d)\left(\operatorname{resp} . \Sigma_{0}(\boldsymbol{V}, d)\right)$ which is isomorphic to a member of $\Sigma$ is in $\Sigma$; (b) When $\boldsymbol{W}_{1}, \boldsymbol{W}_{2}, \boldsymbol{W}_{3}$ are members of $\Sigma(\boldsymbol{V}, d)$ (resp. $\Sigma_{0}(\boldsymbol{V}, d)$ ) such that $W_{1} \rightarrow W_{2}$ ref. $k_{0}, W_{2} \rightarrow W_{3}$ ref. $k_{0}$ and that $W_{1}, W_{3} \in \Sigma$, then $W_{2} \in \Sigma$. When $\Sigma(\boldsymbol{V}, d), \quad \Sigma_{0}(\boldsymbol{V}, d), \quad \Sigma^{\prime}(\boldsymbol{V}, d), \quad \Sigma_{0}^{\prime}(\boldsymbol{V}, d)$ admit universal families, they are admissible subsets of $\Sigma(\boldsymbol{V}, d)$ and $\Sigma_{0}(\boldsymbol{V}, d), \quad \Sigma_{0}^{\prime}(\boldsymbol{V}, d)$ are admissible subsets of $\Sigma_{0}(\boldsymbol{V}, d)$.

THEOREM 5. Let $\boldsymbol{V}^{n}$ be a polarized variety of rank at most $d, \Sigma$ an admissible subset of $\Sigma(\boldsymbol{V}, d)$ (resp. $\Sigma_{0}(\boldsymbol{V}, d)$ ) and assume that $\mathfrak{S}$ is a universal family of $\Sigma$ which is contained in $\Sigma$. Then $\Sigma$ admits a smooth universal family $\mathfrak{F}$, contained in $\Sigma$. If each component of $\mathfrak{K}$ is defined over a field $k$, then $\mathfrak{F}$ can be chosen so that every component of it is also defined over $k$.

Proof. We shall assume that $\Sigma \subset \Sigma(\boldsymbol{V}, d)$ since the other case is 
similar. Let $\mathfrak{M}$ be the type of polarization of $\boldsymbol{V}$. We may assume, without loss of generality, that $\boldsymbol{V}$ is contained in $\Sigma$. We shall identify members of $\mathfrak{S}$ with their underlying varieties. Let $Z$ be a basic polar divisor of $\boldsymbol{V}$ and $Z_{W}$ a basic polar divisor of a member $W$ of $\mathfrak{S}$. By Lemma 5 , there are positive integers $e, e_{W}$ such that $e^{n} Z^{(n)}=e_{W}{ }^{n} Z_{W}^{(n)}$ and $\chi(\boldsymbol{V}, m e Z)=\chi\left(W, m e_{W} Z_{W}\right)$. Moreover, we have $e=e_{W}$ if $\operatorname{rank}(\boldsymbol{V})=\operatorname{rank}(W)$. $\mathfrak{S}$ can be written as a finite union of algebraic families $\mathfrak{S}\left(d_{i}\right)$ such that $\mathfrak{S C}\left(d_{i}\right)$ consists of polarized varieties of rank $d_{i}$ by Proposition 4.3 of the Appendix. Therefore, it is easy to see that $e$ can be fixed throughout $\mathfrak{S}$ and that $e_{W}$ can be taken from a finite set $I$ of positive integers. Let $r_{W}$ be defined by $C_{W} \equiv r_{W} Z_{W} \bmod$ $\mathbb{S}_{a}$. The set $I^{\prime}$ of these $r_{W}$ is finite since $C_{W}{ }^{\left({ }^{n}\right)}$ is bounded. Set $r=\Pi_{I}, r_{W}$. $\left(r / r_{W}\right) e_{W} C_{W} \equiv r e_{W} Z_{W} \bmod \quad \leftrightarrow_{a}$. Then $\chi(\boldsymbol{V}, m r e Z)=\chi\left(W, m\left(r / r_{W}\right) e_{W} C_{W}\right)$ for all $W$ by Proposition 3.2 of the Appendix. Set $T_{W}=\left(r / r_{W}\right) e_{W} C_{W}$ and $g(m)$ $=\chi\left(W, m T_{W}\right)$. Applying Theorem 1 to $W, T_{W}$ and $g(m)$, we see an existence of a constant $c$, depending only on $g($ i.e. on $\mathfrak{S})$ with the following properties: (a) $m T_{W}$ is sufficiently ample on $W$ when $m>c$; (b) $l\left(m T_{W}\right)=g(m)$ $=l(X)=\chi(W, X)$ when $X$ is in $\mathfrak{c}_{a}\left(m T_{W}\right), m>c$. Let us fix $m$ such that $m>c$, $m \notin \mathfrak{M}$.

We reembed every member $W$ of $\mathfrak{S}$ into the projective space of dimension $g(m)-1$ by non-degenerate embeddings determined by divisors in $\mathfrak{c}_{a}\left(m T_{W}\right)$. Images are non-singular subvarieties of degree $m^{n} T_{W}{ }^{\left({ }^{n}\right)}=$ $(m r e)^{n} Z^{(n)}$. We have to analyse the set of images carefully. Let the $\mathfrak{S}_{\alpha}$ be the components of $\mathfrak{H}$ and the $H_{\alpha}$ the Chow-varieties of the $\mathfrak{H}_{\alpha}$. Let $w$ be a generic point of $H_{\alpha}$ over $k$, representing $W . \mathbb{E}_{a}\left(m T_{W}\right)^{+}$is a complete total family defined over $k(w)$ (cf. Proposition 2 and Theorems $E, G$ ). Let $X$ be a generic divisor of this family over $k(w), L$ a field of rationality of $X$ over $k(w)$ and let $\boldsymbol{f}_{X}$ be defined over $L$. Let $\tau$ be a generic projective transformation of the ambient space of $\boldsymbol{f}_{X}(W)$ over $L$ and set $\boldsymbol{f}=\tau \circ \boldsymbol{f}_{X}, \boldsymbol{f}(W)=U$, $\mathfrak{c}(U)=u, t=\mathfrak{c}\left(\Gamma_{\boldsymbol{f}}\right)$. Let $\bar{T}_{\alpha}=\operatorname{loc}_{\bar{k}}(w, u, t), \quad \bar{F}_{\alpha}=\operatorname{loc}_{\bar{k}}(u), \bar{J}_{\alpha}=\operatorname{loc}_{\bar{k}}(t)$. Then $\bar{T}_{\alpha}$, $\bar{F}_{\alpha}, \bar{J}_{\alpha}$ are defined over $k$ by Lemma 4.1 of the Appendix. The set $F_{\alpha}^{*}$ of points in $\bar{F}_{\alpha}$ which represents non-singular varieties, not contained in any hyperplane, is $k$-open on $\bar{F}_{\alpha}$ and the set $J_{\alpha}$ of points on $\bar{J}_{\alpha}$, which represents irreducible cycles in the multiple projective space, is $k$-open on $\bar{J}_{\alpha}$ by the same lemma. Hence $F^{*}=U_{\alpha} F_{\alpha}^{*}$ and $J=U_{\alpha} J_{\alpha}$ are locally closed sets over $k$ in their ambient projective spaces. Let $T_{\alpha}$ be the restriction of $\vec{T}_{\alpha}$ on $H_{\alpha} \times F^{*} \times J_{\alpha}$ and set $T=\cup_{\alpha} T_{\alpha}, H=\cup_{\alpha} H_{\alpha}$. 
We contend that the set-theoretic projection $F$ of $T$ on $F^{*}$ is k-open on $F^{*}$. In order to prove this, we apply Proposition 4.1 of the Appendix, taking $K$ to be $k$. The condition (c) is clearly satisfied. The condition (b) is satisfied by Lemma 4.2 of the Appendix and by the fact that $\mathfrak{H}$ is a universal family of our admissible subset $\Sigma$ of $\Sigma(\boldsymbol{V}, d)$. When $A$ is a subvariety of $F_{\alpha}^{\prime}$, defined over a field $k^{\prime}$ containing $k$, and when a generic point of $A$ is a projection of a point of $T$, then $T^{\prime}=H \times A \times J \cap T$ is a finite union of locally closed subvarieties of the multiple projective space, containing a component $T_{0}^{\prime}$ which has the projection $A$ on $F^{*}$. Hence the projection of $T^{\prime}$ on $A$ contains a non-empty $k^{\prime}$-open subset of $A$ (cf. [24]). Thus (a) is verified and our assertion is proved.

When $\mathfrak{F}$ is the family of polarized varieties of the same type as $\boldsymbol{V}$, determined by $F$, each component of $\mathfrak{F}$ is defined over $k$. Thus $\mathfrak{F}$ is a smooth universal family of $\Sigma$ which is contained in $\Sigma$. q.e.d.

COROLlary. Using the same notations and assumptions as in our theorem, let $\mathfrak{F}$ be a smooth universal family contained in $\Sigma$. Let $\boldsymbol{U}$ be a member of $\mathfrak{F}$ and $\mathfrak{F}_{0}$ a component of $\mathfrak{F}$ containing $\boldsymbol{U}$. Then $\mathfrak{P}\left(\boldsymbol{U}, \mathfrak{S}_{a}\left(C_{\boldsymbol{U}}\right)\right) \subset \mathfrak{F}_{0}$.

Proof. Let $k$ be a field of definition of $\mathfrak{F}_{0}$ and $\boldsymbol{W}$ a generic member of $\mathfrak{F}_{0}$ over $k$. $\mathfrak{E}_{a}\left(C_{\boldsymbol{W}}\right)^{+}$and $\mathfrak{E}_{a}\left(C_{\boldsymbol{U}}\right)^{+}$are complete total families by Proposition 2. Then, when $Y$ is a divisor in $\mathfrak{E}_{a}\left(C_{\boldsymbol{U}}\right)^{+}$, there is a divisor $X$ in $\mathbb{E}_{a}\left(C_{\boldsymbol{W}}\right)^{+}$ such that $(\boldsymbol{W}, X) \rightarrow(\boldsymbol{U}, Y)$ ref. $k$ by Proposition $4 . \quad Y$ and $X$ are sufficiently ample and $l(X)=l(Y)$ by Proposition 3. $\mathfrak{P}\left(\boldsymbol{W}, \mathfrak{C}_{a}\left(C_{\boldsymbol{W}}\right)\right)$ is contained in $\mathfrak{F}$ by the definition of $\mathfrak{F}$ and it has a structure of an algebraic variety by Theorem $E$. It also contains $\boldsymbol{W}$. Hence it is contained in $\mathfrak{F}_{0}$ since it is a component of $\mathfrak{F}$. Then our corollary follows from the compatibility of specializations with algebraic projection and from Proposition 6, applied to $(\boldsymbol{W}, X)$ and $(\boldsymbol{U}, Y)$.

§ 5. Equivalence relations. Let $\mathfrak{F}$ be an algebraic family of polarized varieties in a projective space. By the equivalence relation on $\mathfrak{F}$, we understand always an equivalence relation on $\mathfrak{\mho}$ defined in terms of isomorphisms. Let $F$ be the set of Chow-points of members of $\mathfrak{F}$. The equivalence relation on $\mathfrak{F}$ defines an equivalence relation on $F$, which we shall call the equivalence relation on $F$. We shall say that the equivalence relation thus defined is $k$-closed if $F$ is normally algebraic over $k$ and if the set $\&$ of pairs of equivalent points on $F$ is a $k$-closed subset of $F \times F$. We shall say that it closed if there is a field $k$ such that it is $k$-closed. The equivalence relation 
will be called strongly $k$-closed if it is $k$-closed and if the relation which defines the equivalence of two members of $\mathfrak{\mho}$ is stable by specializations over $k$. By this we understand the following. Let $\boldsymbol{V}, \boldsymbol{W}, \boldsymbol{V}^{\prime}, \boldsymbol{W}^{\prime}$ be members of $\mathfrak{F}$ such that $\boldsymbol{V}$ and $\boldsymbol{W}$ are isomorphic by an isomorphism $\boldsymbol{f}$ and that $(\boldsymbol{V}, \boldsymbol{W})$ $\rightarrow\left(\boldsymbol{V}^{\prime}, \boldsymbol{W}^{\prime}\right)$ ref. k. Then an extension $\Gamma_{\boldsymbol{f}} \rightarrow \Gamma^{\prime}$ ref. $k$ of the specialization to $\Gamma_{\boldsymbol{f}}$ is such that $\Gamma^{\prime}$ is the graph of an isomorphism between $\boldsymbol{V}^{\prime}$ and $\boldsymbol{W}^{\prime}$. When there is a field $k$ such that the equivalence relation is strongly $k$ closed, we shall say that it is strongly closed.

THEOREM 6. Let $\boldsymbol{V}$ be a non-ruled polarized variety of rank at most $d$ and $\Sigma$ an admissible subset of $\Sigma(\boldsymbol{V}, d)$, containing $\boldsymbol{V}$. Let $\mathfrak{F}$ a smooth universal family of $\Sigma$ which is contained in $\Sigma$. Then there is an algebraic family $\mathfrak{B}$ of polarized varieties with the following properties:

(a) The set of Chow-points of members of $\mathfrak{B}$ is a closed subset of that of $\mathfrak{F}$;

(b) $\mathfrak{B}$ is a subset of $\mathfrak{F}$ and is stable by the equivalence relation on $\mathfrak{F}$;

(c) A non-ruled member of $\Sigma$ is isomorphic to a member of $\mathfrak{F}-\mathfrak{B}$;

(d) The equivalence relation on $\mathfrak{F}$ induces on $\mathfrak{F}-\mathfrak{B}$ a strongly closed equivalence relation.

Proof. Let $F$ be the set of Chow-points of members of $\mathfrak{F}$. Let $k$ be an algebraically closed common field of definition of the components of $F$. Express $F$ as a union of components as follows: $F=\left(\cup_{\alpha} F_{\alpha}\right) \cup\left(\cup_{\beta} F_{\beta}\right)$, where generic points of the $F_{\alpha}$ (resp. $F_{\beta}$ ) over $k$ represent non-ruled (resp. ruled) varieties. $F_{\alpha}$ exists by Theorem 1.1 of the Appendix.

Step I. We shall identify polarized varieties with their underlying varieties for the sake of simplicity. Let $u$ be a generic point of $F_{\alpha}$ over $k$, representing $U$. By the definition of $\mathfrak{F}, C_{U}$ is sufficiently ample. Then $\mathfrak{r}_{a}\left(C_{U}\right)^{+}$ is a complete total family defined over $k(u)$ by Proposition 2 and Theorem $G$. Let $X$ be a generic divisor of it over $k(u), K$ a field of rationality of $X$ over $k(u)$ and $\boldsymbol{f}=\boldsymbol{f}_{X}$ a non-degenerate projective embedding of $U$, determined by $X$, defined over $K$. Let $\tau$ be a generic projective transformation over $K$ of the ambient space of $\boldsymbol{f}(U)$ and set $W=\tau \circ \boldsymbol{f}(U)$. Let $w=\mathfrak{c}(W)$, $t=\mathfrak{c}(\Gamma)$ where $\Gamma=\Gamma_{\tau} \circ \boldsymbol{f}, \boldsymbol{P}$ the ambient projective space of the locus of $t$ over $k$ and the $A_{\alpha}$ the locus of $(t, u, w)$ over $k$ on $\boldsymbol{P} \times F \times F$. Let $A$ be the union of the $A_{\alpha}$, which is clearly closed on $\boldsymbol{P} \times F \times F$. Denote by $B^{\prime}$ the set of points $\left(t^{\prime}, u^{\prime}, w^{\prime}\right)$ in $A$ such that $t^{\prime}$ represents a reducible cycle. $B^{\prime}$ 
is a proper closed subset of $A$. Let $B$ be the closure on $F$ of the set-theoretic projection of $B^{\prime}$ on the second factor of the product $\boldsymbol{P} \times F \times F . \quad B$ defines an algebraic family of polarized varieties. We shall show that this family satisfies our requirements.

Step II. A point $\left(t^{\prime}, u^{\prime}, w^{\prime}\right)$ is in $A_{\alpha}-B^{\prime}$ if and only if $u^{\prime}$ represents a member $U^{\prime}$ of $F_{\alpha}, t^{\prime}$ represents a non-degenerate projective embedding $\boldsymbol{f}_{X^{\prime}}$ of $U^{\prime}$ with $X^{\prime} \in \mathbb{C}_{a}\left(C_{U^{\prime}}\right)^{+}$and $w^{\prime}$ represents $W^{\prime}=\boldsymbol{f}_{X^{\prime}}\left(U^{\prime}\right)$.

This follows from Lemma 4.2 of the Appendix.

Step III. (a) is clearly satisfied by $B$. In order to prove (c), it is enough to show that a generic point $u^{\prime}$ of a component of $B$ over $k$ represents a ruled variety by virtue of Theorem 1.1 of the Appendix. There is a generic point $\left(t^{\prime}, u^{\prime}, w^{\prime}\right)$ of a component of $B^{\prime}$ over $k$. When it is in $A_{\alpha}$, it is a specialization of $(t, u, w)$ over $k$. Since $u^{\prime}, w^{\prime}$ represents members of $\mathfrak{F}$ and since $t^{\prime}$ represents a reducible cycle, $u^{\prime}, w^{\prime}$ must represent ruled varieties by Theorem $D$ and by Propositions 2 and 4 .

Next we shall prove (b). Let $u^{\prime \prime} \in B, u^{\prime \prime *} \in F$ representing $U^{\prime \prime}, U^{\prime \prime *}$, such that $U^{\prime \prime *}$ is isomorphic to $U^{\prime \prime}$ by an isomorphism $\boldsymbol{h}^{\prime \prime}$ (as polarized varieties). Let $u^{\prime}$ be a generic point of a component of $B$ over $k$ such that $u^{\prime} \rightarrow u^{\prime \prime}$ ref. $k$. By Propositions 4 and 6 and by the definition of $F$, there is a point $u^{\prime *}$ in $F$, representing $U^{\prime *}$, and an isomorphism $\boldsymbol{h}^{\prime}$ between $U^{\prime *}$ and $U^{\prime}$, which is represented by $u^{\prime}$, such that $\left(U^{\prime}, U^{\prime *}, \Gamma_{\boldsymbol{h}^{\prime}}\right) \rightarrow\left(U^{\prime \prime}, U^{\prime \prime *}, \Gamma_{\boldsymbol{h}^{\prime \prime}}\right)$ ref. $k$. Therefore, it is enough to prove that $u^{\prime *}$ is in $B$ since $B$ is $k$-closed on $F$. Since $u^{\prime}$ is a generic point of a component of $B$ over $k$, there is a point $\left(t^{\prime}, u^{\prime}, w^{\prime}\right)$ of $B^{\prime}$. Without loss of generality, we may assume that it in $A_{\alpha}$. Since $(t, u, w) \rightarrow\left(t^{\prime}, u^{\prime}, w^{\prime}\right)$ ref. $k$, there is a discrete valuation-ring $\mathfrak{D}$ of $k(t, u, w)$ such that $(t, u, w) \rightarrow\left(t^{\prime}, u^{\prime}, w^{\prime}\right)$ ref. 5 . Then $u \rightarrow u^{\prime}$ ref. $\mathfrak{D}$, i.e. $U \rightarrow U^{\prime}$ ref. 5 . Applying Proposition 6 to this and to $U^{\prime *}, \boldsymbol{h}^{\prime}$, we see the existence of a member $U^{*}$ of $\mathfrak{F}$ and an isomorphism $\boldsymbol{h}$ of $U^{*}$ and $U$ such that $\left(U, U^{*}, \Gamma_{\boldsymbol{h}}\right) \rightarrow\left(U^{\prime}, U^{\prime *}, \Gamma_{\boldsymbol{h}^{\prime}}\right)$ ref. $\mathfrak{D} . \quad \boldsymbol{g}=\boldsymbol{\tau} \circ \boldsymbol{f} \circ \boldsymbol{h}^{-1}$ is an isomorphism between $U^{*}$ and $W$, and we see easily that an extension $\Gamma_{g} \rightarrow \Gamma^{* *}$ ref. $\mathfrak{D}$ of the above specialization over $\mathfrak{D}$ is such that $\Gamma^{*}$ is a reducible cycle on $U^{\prime *} \times W^{\prime}$. Set $\mathfrak{c}\left(U^{*}\right)=u^{*}, \mathfrak{c}\left(\Gamma_{\boldsymbol{g}}\right)=\tilde{t}$ and $\mathfrak{c}\left(\Gamma^{*}\right)=\tilde{t}^{\prime}$. Then $\left(\tilde{t}, u^{*}, w\right) \rightarrow\left(\tilde{t}^{\prime}, u^{\prime} *, w^{\prime}\right)$ ref. $k$. Since $U=h(U *)$ and since $U *$ is a member of $\mathfrak{F}, u^{*}$ is a point of $\mathfrak{F}_{\alpha}$ by our choice of $u$ (cf. Corollary to Theorem 5). Then $\left(\tilde{t}, u^{*}, w\right) \in A_{\alpha}$ by the result of Step II. When that is so, $\left(\tilde{t}^{\prime}, u^{\prime *}, w^{\prime}\right) \in A_{\alpha}$ and consequently it is in $B^{\prime}$ since 
$t^{\prime}$ represents a reducible cycle. (b) is thus proved.

Finally we shall prove (d). Let $u^{\prime}, w^{\prime}$ be two points of $F$, representing two isomorphic varieties $U^{\prime}, W^{\prime}$, isomorphic by the map $h^{\prime}$. If $u^{\prime} \in F_{\alpha}$, then $(\Gamma, U, W) \rightarrow\left(\Gamma_{\boldsymbol{h}^{\prime}}, U^{\prime}, W^{\prime}\right)$ ref. $k$ by the result of step II. Hence $\left(u^{\prime}, w^{\prime}\right)$ is in the projection of $A$ on $F \times F$.

Let $u^{\prime \prime}, w^{\prime \prime}$ be points of $F-B$, representing $U^{\prime \prime}, W^{\prime \prime}$ and $u^{\prime}, w^{\prime}$ be now points of $F$, representing $U^{\prime}, W^{\prime}$ and assume that $U^{\prime}, W^{\prime}$ are isomorphic by an isomorphism $h^{\prime}$ and that $\left(U^{\prime}, W^{\prime}\right) \rightarrow\left(U^{\prime \prime}, W^{\prime \prime}\right)$ ref. $k$. Assume that $u^{\prime}$ is in $F_{\alpha}$. Then $(\Gamma, U, W) \rightarrow\left(\Gamma_{\boldsymbol{h}^{\prime}}, U^{\prime}, W^{\prime}\right)$ ref. $k$ by step II. When $\left(\Gamma_{\boldsymbol{h}^{\prime}}, U^{\prime}, W^{\prime}\right)$ $\rightarrow\left(\Gamma^{\prime \prime}, U^{\prime \prime}, W^{\prime \prime}\right)$ ref. $k, \quad\left(\Gamma^{\prime \prime}, U^{\prime \prime}, W^{\prime \prime}\right)$ is a specialization of $(\Gamma, U, W)$ over $k$. Hence $\Gamma^{\prime \prime}$ is irreducible by the choice of $U^{\prime \prime}$. Then $\Gamma^{\prime \prime}$ is the graph of an isomorphism between $U^{\prime \prime}$ and $W^{\prime \prime}$ by Theorem $D^{\prime}$, Propositions 2,4 and by the sufficient ampleness of hyperplane sections. (d) follows from these observations at once.

Remark 5.1. Theorem 6 is valid to $\Sigma_{0}(\boldsymbol{V}, d)$ too.

Remark 5.2. A non-singular surface in a projective space is ruled if and only if $P_{12}=0$ according to the theorem of Enriques-Kodaira, the first complete proof of which was given by Kodaira in 1961 (unpublished). According to an unpublished work of Mumford, it is so if and only if $P_{m}=0$ for all positive $m$ for characteristic $p$. When we combine this with our Theorem 1.1 and Proposition 2.1 of the Appendix, every member of $\Sigma(\boldsymbol{V})$ is a ruled surface if and only if $\boldsymbol{V}$ is a ruled surface. Therefore, if $\boldsymbol{V}$ is a non-ruled surface, $\Sigma(\boldsymbol{V}, d)$ admits a universal family and $\mathfrak{B}$ in our theorem is empty. When $\operatorname{dim} V>2$, every little seems to be known in this line. Therefore, we shall mention here some problems, affirmative solutions of which would enlighten the situation.

Let $V$ and $V^{\prime}$ be complete non-singular varieties such that $V^{\prime}$ is a specialization of $V$ over some discrete valuation-ring. If $V$ is not a ruledvariety, is it true that $V^{\prime}$ is not a ruled variety?

When $V$ is a ruled variety, all the $P_{m}$ vanish at least in the case of characteristic 0 . Is this fact invariant of the deformations?

Since the general situations are as above, we shall introduce the following definition. Let $\boldsymbol{V}$ be a polarized variety of rank at most $d$ and $\Sigma$ an admissible subset of $\Sigma(\boldsymbol{V}, d)$. Let $\boldsymbol{U}$ be a member of $\Sigma$ and assume that there is a smooth universal family $\mathfrak{F}$ of $\Sigma$ contained in $\Sigma$ and an algebraic 
family $\mathfrak{B}$ of polarized varieties, which satisfies the four conditions of Theorem 6 such that $\boldsymbol{U}$ is isomorphic to a member of $\mathfrak{F}-\mathfrak{B}$. Then we shall say that $\boldsymbol{U}$ is a solid polarized variety of $\Sigma$. We shall use the similar definitions for admissible subsets of $\Sigma_{0}(\boldsymbol{V}, d)$. Let $\mathfrak{F}$ be a smooth universal family of $\Sigma$ contained in $\Sigma$ and $\mathfrak{B}$ an algebraic family of polarized varieties, satisfying the four conditions of Theorem 6 , such that a solid polarized variety of $\Sigma$ is always isomorphic to a member of $\mathfrak{F}-\mathfrak{B}$. Then we shall say that $\mathfrak{F}-\mathfrak{B}$ is a typical universal family of $\Sigma$.

Proposition 9. Let $\boldsymbol{V}$ be a non-ruled polarized variety of rank at most $d$ and $\Sigma$ an admissible subset of $\Sigma(\boldsymbol{V}, d)$ (resp. $\left.\Sigma_{0}(\boldsymbol{V}, d)\right)$, containing $\boldsymbol{V}$. Let $\mathfrak{F}$ and $\mathfrak{F}^{\prime}$ be two smooth universal families of $\Sigma, \boldsymbol{U}$ and $\boldsymbol{U}^{\prime}$ be members of $\mathfrak{F}$ and $\mathfrak{F}^{\prime}$ respectively and assume that they are isomorphic by an isomorphism $\boldsymbol{g}^{\prime}$. Let $k$ be a common field of definition of the components of $\mathfrak{F}, \mathfrak{F}^{\prime}$ and consider the following property:

(Wet $\boldsymbol{L}, \boldsymbol{M}, \boldsymbol{W}$ be members of $\mathfrak{F}$ such that $\boldsymbol{L}$ and $\boldsymbol{M}$ are isomorphic by an isomorphism $\boldsymbol{f}$ and that $(\boldsymbol{L}, \boldsymbol{M}) \rightarrow(\boldsymbol{U}, \boldsymbol{W})$ ref. $k$. When $\Gamma^{\prime}$ is a specialization of $\Gamma_{\boldsymbol{f}}$ over $k$ over the specialization above, $\Gamma^{\prime}$ is the graph of an isomorphism between $\boldsymbol{U}$ and $\boldsymbol{W}$.

When (㮖) denotes the corresponding property for $\boldsymbol{U}^{\prime}$, replacing $\mathfrak{F}$ by $\mathfrak{F}^{\prime}$, then (\$) and (w) are equivalent.

Proof. Assume that $\boldsymbol{U}^{\prime}$ satisfies (\#). By Propositions 6,7 and by Theorem 5, there are members $\boldsymbol{L}^{\prime}, \boldsymbol{M}^{\prime}, \boldsymbol{W}^{\prime}$ of $\mathfrak{F}^{\prime}$ and isomorphisms $\boldsymbol{g}$ between $\boldsymbol{L}$ and $\boldsymbol{L}^{\prime}, \boldsymbol{h}$ between $\boldsymbol{M}$ and $\boldsymbol{M}^{\prime}$ and $\boldsymbol{h}^{\prime}$ between $\boldsymbol{W}$ and $\boldsymbol{W}^{\prime}$ such that $\left(\boldsymbol{L}^{\prime}, \boldsymbol{M}^{\prime}, \Gamma_{\boldsymbol{g}}, \Gamma_{\boldsymbol{h}}\right) \rightarrow\left(\boldsymbol{U}^{\prime}, \boldsymbol{W}^{\prime}, \Gamma_{\boldsymbol{g}^{\prime}}, \Gamma_{\boldsymbol{h}^{\prime}}\right) \quad$ ref. $k$ over the specialization $\left(\boldsymbol{L}, \boldsymbol{M}, \Gamma_{\boldsymbol{f}}\right) \rightarrow$ $\left(\boldsymbol{U}, \boldsymbol{W}, \Gamma^{\prime}\right)$ ref. $k$. When we extend our specializations further to a specialization of $\Gamma_{\boldsymbol{h f g}-1}$ over $k$, it specializes to the graph of an isomorphism between $\boldsymbol{U}^{\prime}$ and $\boldsymbol{W}^{\prime}$ by (Hence $I^{\prime}$ is easily seen to be the graph of an isomorphism between $\boldsymbol{U}$ and $\boldsymbol{W}$. Thus $\boldsymbol{U}$ satisfies (\#). In the same way, (\#) implies (耕).

Proposition 10. Let $\boldsymbol{V}$ be a non-ruled polarized variety of type $\mathfrak{M}$ and of rank at most $d$. Let $\Sigma$ be an admissible subset of $\Sigma(\boldsymbol{V}, d)$, containing $\boldsymbol{V}, \mathfrak{F} a$ smooth universal family of $\Sigma$ and $\mathfrak{B}$ an algebraic family of polarized varieties which satisfies the four conditions of Theorem 6 relative to $\mathfrak{F}$. Let $s$ be a positive integer not in $\mathfrak{M}$. Let $\left(\mathfrak{F}_{r}, \mathfrak{B}_{r}\right)$ be either one of the following (a), (b): (a) $r=s, \mathfrak{F}_{r}=$ $\cup_{\boldsymbol{U} \in \mathfrak{F}} \mathfrak{P}\left(\boldsymbol{U}, \mathfrak{C}_{a}\left(r C_{\boldsymbol{U}}\right)\right)$ and $\mathfrak{B}_{r}=\cup_{\boldsymbol{U} \in \mathfrak{B}}\left(\boldsymbol{U}, \mathfrak{F}_{a}\left(r C_{\boldsymbol{U}}\right)\right)$; (b) $r=1 / s$ and $\mathfrak{F}_{r}, \mathfrak{B}_{r}$ as in (a) if every $\boldsymbol{U}$ in $\mathfrak{F}$ contains a sufficiently ample divisor $X$ such that $s X \equiv C_{\boldsymbol{U}} \bmod$ 
$\mathscr{S}_{a}$. Then $\mathfrak{F}_{r}$ is a smooth universal family of $\Sigma$ and $\mathfrak{B}_{r}$ is an algebraic family, satisfying the four conditions of Theorem 6 relative to $\mathfrak{F}_{r}$.

Proof. Since $\mathfrak{F}$ is a smooth universal family, $\mathfrak{F}, \mathfrak{B}$ and $r$ satisfy the requirements of the Corollary of Proposition 4.2 of the Appendix (cf. also Corollary to Theorem 5). Therefore, $\mathfrak{F}_{r}$ and $\mathfrak{B}_{r}$ are algebraic families of polarized varieties of type $\mathfrak{M}$ such that the hyperplane sections are polar divisors. From the definition of $\mathfrak{F}_{r}$, it is clear that $\mathfrak{F}_{r}$ is a smooth universal family. When $k$ is a common field of definition of the components of $\mathfrak{F}, \mathfrak{B}, \mathfrak{F}_{r}, \mathfrak{B}_{r}$ and when $\boldsymbol{A}$ is a member of $\mathfrak{B}_{r}$, a specialization $\boldsymbol{A}^{\prime}$ of $\boldsymbol{A}$ over $k$ in $\mathfrak{F}_{r}$ is also a member of $\mathfrak{B}_{r}$. This follows from the definition of $\mathfrak{B}_{r}$, (a) of Theorem 6 (satisfied by $\mathfrak{B}$ ) and from Proposition 7. Therefore, the set of Chow-points of members of $\mathfrak{B}_{r}$ is closed on that of $\mathfrak{F}_{r}$. From the definition of $\mathfrak{B}_{r}$, it is clearly closed by the equivalence relation on $\mathfrak{F}_{r}$ and a non-ruled member of $\Sigma$ is isomorphic to a member of $\mathfrak{F}_{r}-\mathfrak{B}_{r}$.

Finally, we shall show that the equivalence relation on $\mathfrak{\mho}_{r}$ induces on $\mathfrak{F}_{r}-\mathfrak{B}_{r}$ a strongly closed equivalence relation. Let $\boldsymbol{W}$ be a generic member of a component of $\mathfrak{F}_{r}$ over $k$ and $K$ a field of definition of $\mathfrak{p}\left(\boldsymbol{W}, \mathfrak{c}_{a}\left(C_{\boldsymbol{W}}\right)\right)$ over $k$. Let $\boldsymbol{W}^{*}$ be a generic member of $\mathfrak{B}\left(\boldsymbol{W}, \mathfrak{C}_{a}\left(C_{\boldsymbol{W}}\right)\right)$ over $K . \boldsymbol{W}^{*}$ is contained in $\widetilde{F}_{r}$ by the definition of a smooth universal family. Let $\boldsymbol{W}^{\prime}$ be a member of $\mathfrak{F}_{r}$, contained in the same component of $\mathfrak{F}_{r}$ as $\boldsymbol{W}$ and $\boldsymbol{W}^{\prime *}$ a member of $\mathfrak{F}_{r}$ which is isomorphic to $\boldsymbol{W}^{\prime}$. There is a member $\tilde{\boldsymbol{W}}$ of $\mathfrak{B}\left(\boldsymbol{W}, \mathfrak{C}_{a}\left(C_{\boldsymbol{W}}\right)\right)$ such that $(\boldsymbol{W}, \tilde{\boldsymbol{W}}) \rightarrow\left(\boldsymbol{W}^{\prime}, \boldsymbol{W}^{\prime *}\right)$ ref. $k$ by Proposition 6. By Proposition 2 and by Theorems $E$ and $G$, we may take $K$ to be a field of definition of $\boldsymbol{W}$ over $K$. When this is done, $(\boldsymbol{W}, \tilde{\boldsymbol{W}})$ is a specialization of $\left(\boldsymbol{W}, \boldsymbol{W}^{*}\right)$ over $K$. Hence $\left(\boldsymbol{W}, \boldsymbol{W}^{*}\right) \rightarrow\left(\boldsymbol{W}^{\prime}, \boldsymbol{W}^{*}\right)$ ref. $k$. Let $F_{r}$ be the set of Chow-points of members of $\mathfrak{F}_{r}$. Then the above shows that the set of pairs of equivalent points on $F_{r}$ is contained in a $k$-closed subset of $F_{r} \times F_{r}$ such that generic points of the components of the closed subset over $\bar{k}$ are pairs of equivalent points. When that is so, our assertion follows easily from Propositions 6 and 9 and from (d) of Theorem 6 which is satisfied by $\mathfrak{B}$.

THEOREm 7. Let $\boldsymbol{V}$ be a non-ruled polarized variety of rank at most $d$ and $\Sigma$ an admissible subset of $\Sigma(\boldsymbol{V}, d)$, containing $\boldsymbol{V}$. Then $\Sigma$ admits a typical universal family. When $\mathfrak{F}$ is a smooth universal family of $\Sigma$, contained in $\Sigma$, and $\mathfrak{B}$ 
the intersection of algebraic families which satisfy the conditions of Theorem 6 relative to $\mathfrak{F}, \mathfrak{F}-\mathfrak{B}$ is a typical universal family contained in $\Sigma$.

Proof. It is easy to verify that $\mathfrak{B}$ satisfies also the conditions of Theorem 6. Let $\boldsymbol{U}$ be a solid polarized variety of $\Sigma$. By our definition, there is a smooth universal family $\mathfrak{F}^{\prime}$ contained in $\Sigma$ and an algebraic family $\mathfrak{B}^{\prime}$, satisfying the conditions of Theorem 6, such that $\boldsymbol{U}$ is isomorphic to a member of $\mathfrak{F}^{\prime}-\mathfrak{B}^{\prime}$. Let $\mathfrak{M}$ be the type of polarization of $\boldsymbol{V}$ and $r$ a positive integer not in $\mathfrak{M}$. Define $\mathfrak{B}_{r}^{\prime}, \mathfrak{F}_{r}^{\prime}$ as in Proposition $10,(\mathrm{a}) . \mathfrak{F}_{r}^{\prime}$ is a smooth universal family, $\mathfrak{B}_{r}^{\prime}$ satisfies the conditions of Theorem 6 relative to $\mathfrak{F}_{r}^{\prime}$ and $\boldsymbol{U}$ is isomorphic to a member of $\mathfrak{F}_{r}^{\prime}-\mathfrak{B}_{r}^{\prime}$. From the definitions, it is possible to find a pair $(r, u)$ of positive integers, not contained in $\mathfrak{M}$, such that $\mathfrak{F}_{r}^{\prime}=\mathfrak{F}_{u}$ where $\mathfrak{F}_{u}$ is defined as in Proposition $10,(\mathrm{a})$. When $\mathfrak{B}_{u}$ is defined similarly, our theorem will be proved if we show that $\mathfrak{B}_{u}$ is the intersection of algebraic families which satisfy the conditions of Theorem 6 relative to $\mathfrak{F}_{u}$. This is because $\mathfrak{B}$ satisfies those conditions relative to $\mathfrak{F}$ and because of Proposition 10, since this implies that $\mathfrak{B}_{u}$ satisfies also those conditions relative to $\mathfrak{F}_{u}$.

Let now $\mathfrak{B}^{\prime}$ be the intersection of all those algebraic families of polarized varieties which satisfy the conditions of Theorem 6 relative to $\mathfrak{F}_{u}$. As we remarked, $\mathfrak{B}^{\prime}$ also satisfies those conditions relative to $\mathfrak{F}_{u}$. Set $t=1 / u$. When we apply Proposition 10,(a) and (b), we get $\left(\mathfrak{F}_{u}\right)_{t}=\mathfrak{F}$ and $\left(\mathfrak{B}_{u}\right)_{t}=\mathfrak{B}$ and $\mathfrak{B}_{t}^{\prime}$ is contained in $\mathfrak{B}$; moreover $\mathfrak{B}_{t}^{\prime}$ satisfies the conditions of Theorem 6 relative to $\mathfrak{F}$. It follows that $\mathfrak{B}_{t}^{\prime}=\mathfrak{B}$ and consequently $\mathfrak{B}^{\prime}=\left(\mathfrak{B}_{t}^{\prime}\right)_{u}=\mathfrak{B}_{u}$. Our theorem is thereby proved.

Remark 5.3. Proposition 10 and Theorem 7 are valid for $\Sigma_{0}(\boldsymbol{V}, d)$ too. Before we discuss the significance of a typical universal family, we have to settle the following proposition.

Proposition 11. Let $\boldsymbol{V}$ (resp. $\boldsymbol{V}^{\prime}$ ) be a polarized variety, $X$ (resp. $X^{\prime}$ ) a sufficiently ample polar divisor of $\boldsymbol{V}$ (resp. $\boldsymbol{V}^{\prime}$ ) and $\subseteq$ a discrete valuation-ring such that $\boldsymbol{V}$ and $X$ are rational over its quotient field and that $(\boldsymbol{V}, X) \rightarrow\left(\boldsymbol{V}^{\prime}, X^{\prime}\right)$ ref. $\mathfrak{S}$. When we denote by $A, A^{\prime}$ the closures of $\mathfrak{p}\left(\boldsymbol{V}, \mathfrak{\Im}_{a}(X)\right), \mathfrak{p}\left(\boldsymbol{V}^{\prime}, \mathfrak{c}_{a}\left(X^{\prime}\right)\right)$ in their ambient projective spaces and when $A \rightarrow L$ ref. $D$, every component of $A^{\prime}$ is contained in a component of $L$.

Proof. Let $\boldsymbol{W}^{\prime}$ be a member of $\mathfrak{B}\left(\boldsymbol{V}^{\prime}, \mathfrak{F}_{a}\left(X^{\prime}\right)\right)$ and $W^{\prime}$ its underlying 
variety. Denote also by $V, V^{\prime}$ the underlying varieties of $\boldsymbol{V}, \boldsymbol{V}^{\prime}$. There is a divisor $Y^{\prime}$ in $\mathfrak{c}_{a}\left(X^{\prime}\right)$ such that $W^{\prime}=\boldsymbol{f}_{Y^{\prime}}\left(V^{\prime}\right)$. Since $X$ and $X^{\prime}$ are sufficiently ample, there is a divisor $Y$ in $\mathfrak{C}_{a}(X)$ such that $(A, Y) \rightarrow\left(L, Y^{\prime}\right)$ ref. 5 by Propositions 2 and 4. Moreover, we have $l(Y)=l\left(Y^{\prime}\right)$ by Proposition 3. Therefore, there is a $\boldsymbol{f}_{Y}$ such that $\left(V, X, A, Y, \Gamma_{\boldsymbol{f}_{Y}}\right) \rightarrow\left(V^{\prime}, X^{\prime}, L, Y^{\prime}, \Gamma_{\boldsymbol{f}_{Y^{\prime}}}\right)$ ref. $\mathbb{D}$ by Proposition 6 (replace $\subseteq$ by a discrete valuation-ring dominating it, if necessary). When that is so, $\boldsymbol{f}_{Y^{\prime}}\left(V^{\prime}\right)=W^{\prime}$ is such that its Chow-point is in $L$ by virtue of the compatibility of specializations with algebraic projection. Our proposition is thereby proved.

COROLlary. Using the same notations and assumptions as in the proposition, assume further that $\boldsymbol{V}^{\prime}$ satisfies the following condition: When $\boldsymbol{W}$ is a member of $\mathfrak{P}\left(\boldsymbol{V}, \mathfrak{S}_{a}(X)\right), \quad \boldsymbol{\Gamma}$ the graph of an isomorphism between $\boldsymbol{V}$ and $\boldsymbol{W}, \boldsymbol{W}^{\prime}$ a polarized variety which is not contained in a hyperplane and when $(\boldsymbol{V}, \boldsymbol{W}, \Gamma) \rightarrow\left(\boldsymbol{V}^{\prime}, \boldsymbol{W}^{\prime}, \Gamma^{\prime}\right)$ ref. $\mathfrak{D}$, then $\Gamma^{\prime}$ is the graph of an isomorphism. Then $L-\mathfrak{p}\left(\boldsymbol{V}^{\prime}, \mathfrak{C}_{a}\left(X^{\prime}\right)\right)$ consists of the Chow-points of those positive cycles which are either singular or contained in a hyperplane and every component of $A^{\prime}$ is a component of $L . \quad$ Moreover, aut $(\boldsymbol{V})$ and aut $\left(\boldsymbol{V}^{\prime}\right)$ are algebraic groups of the same dimension.

Proof. Let us use the notations and conventions of the proof of our proposition. Let $W^{\prime}$ be a non-singular subvariety of a projective space, not contained in a hyperplane, such that $\mathfrak{c}\left(W^{\prime}\right) \in L$. There is a member $W$ of $\mathfrak{P}\left(V, \mathfrak{C}_{a}(X)\right)$ such that $(V, X, A, W) \rightarrow\left(V^{\prime}, X^{\prime}, L, W^{\prime}\right)$ ref. 5 . Let $\boldsymbol{f}$ be an isomorphism between $V$ and $W$ determined by a divisor $Y$ in $\mathfrak{C}_{a}(X)$ and $\left(\Gamma_{f}, Y\right) \rightarrow\left(\Gamma^{\prime}, Y^{\prime}\right)$ ref. $\mathfrak{D}$ be an extension of the above specialization. By our assumption, $\Gamma^{\prime}$ is the graph of an isomorphism between $V^{\prime}$ and $W^{\prime} . Y^{\prime}$ is in $\mathfrak{C}_{a}\left(X^{\prime}\right)$ since algebraic equivalence is preserved by specialization. Hence $Y^{\prime}$ is sufficiently ample and $\Gamma^{\prime}$ is a non-degenerate projective embedding determined by $Y^{\prime}$ by virtue of Proposition 3, since specializations are compatible with intersection-product and algebraic projection. Thus $W^{\prime}$ is a member of $\mathfrak{P}\left(V^{\prime}, \mathfrak{C}_{a}\left(X^{\prime}\right)\right)$. From this and from our proposition, the first and the second assertions follow immediately. The last assertion follows from the above results, Theorem $B$, Proposition 3 and from the Corollary of Theorem $F$.

Remark 5.4. Let $\boldsymbol{V}$ be non-ruled and $\Sigma$ an admissible subset of $\Sigma(\boldsymbol{V}, d)$ (resp. $\Sigma_{0}(\boldsymbol{V}, d)$ ), containing $\boldsymbol{V}$. Then it admits a typical universal family contained in $\Sigma$ by Theorem 7. Let $F$ be the set of Chow-points of 
members of $\mathfrak{F}$ and $E$ the set of pairs of equivalent points on $F$. $E$ is closed on $F \times F$ and when $w$ is a point of $F$, representing $W$, then $w \times F \cap E=w \times\left|\mathfrak{p}\left(\boldsymbol{W}, \mathfrak{S}_{a}\left(C_{\boldsymbol{W}}\right)\right)\right|$ by the definition of equivalence relation. Let $k$ be a common field of definition of the components of $F$ and $E, u$ a point of $F$ representing $\boldsymbol{U}$ and assume that $u \rightarrow w$ ref. $k$. Then $\left|\mathfrak{p}\left(\boldsymbol{U}, \widetilde{c}_{a}\left(C_{U}\right)\right)\right|$ has the unique specialization $\left|\mathfrak{p}\left(\boldsymbol{W}, \mathfrak{S}_{a}\left(C_{\boldsymbol{W}}\right)\right)\right|$ over $k$, over this specialization. These follow from the definitions, Theorem 7 and from Proposition 11.

$\S 6$. Q-varieties of moduli. We shall here briefly review the concept of $Q$-varieties. For details, see [15]. Let $U$ be an abstract variety and $E$ a closed subset of $U \times U$. Assume that every component of $E$ has the projection $U$ on either side of the product and that the components of $E$ have the same dimension. Moreover, assume that $E$ defines an equivalence relation on $U$. When $E$ satisfies these conditions, we shall say that a field $k$ is a field of rationality of $(U, E)$ or a field of definition of it if $U$ is defined over $k, E$ is $k$-closed on $U \times U$ and if every component of $E$ is defined over a separable extension of $k$. We shall say that $(U, E)$ is an unmixed equivalence pair if it satisfies further the following conditions:

$\left(E_{1}\right)$ Let $x$ be a generic point of $U$ over $k, x^{\prime}$ a point of $U$ and $E\{x\}$ (resp. $\left.E\left\{x^{\prime}\right\}\right)$ the set of points of $U$ which are equivalent to $x$ (resp. $x^{\prime}$ ). Then $E\left\{x^{\prime}\right\}$ is the unique specialization of $E\{x\}$ over $k$, over the specialization $x \rightarrow x^{\prime}$ ref. $k$;

$\left(E_{2}\right)$ When $x$ is a generic point of $U$ over $k$, every component of $E\{x\}$ is defined over a separable extension of $k(x)$.

Let $\mathscr{U}$ be the abstract quotient space of $U$ by the equivalence relation defined by $E$ if $(U, E)$ is an unmixed equivalence pair. Let $f$ be the abstract canonical morphism of $U$ on $\mathscr{U}$. We defined on $\mathscr{U}$ the concept of a $Q$-variety, and, with this structure, $f$ is a rational map of $U$ on $\mathscr{U}$. We have shown that $(\mathscr{U}, f)$ can be treated as if it is a pair of an abstract variety and a rational map in many cases from the qualitative point of view in algebraic geometry. When the $E_{\imath}$ are components of $E$, we set $\widetilde{E}=\sum_{i} E_{i}$ where each $E_{i}$ appears with the coefficient 1 . When $x^{\prime}$ is simple on $U, f\left(x^{\prime}\right)$ is called a regular point of $\mathscr{U}$. When $\widetilde{E}\left(x^{\prime}\right)$, defined as usual by the intersection-product, contains a component whose coefficient is prime to the characteristic $p$ of the universal domain, $f\left(x^{\prime}\right)$ is called a p-regular point of $\mathscr{U}$. We have shown that a $p$-regular point can be treated as if it is a 
simple point on an algebraic variety from quantitative point of view in algebraic geometry. If $\mathscr{U}$ consists of $p$-regular points, it is called a $Q$ manifold. When $(U, E)$ is defined over $k, k$ is called a field of definition or rationality of $\mathscr{U}$ and $U$ is called a covering variety of $\mathscr{U}$.

For the sake of convenience, we shall introduce the following definition. Let $\mathscr{T}$ be a topological space. We shall say that $\mathscr{T}$ is a $Q$-space if the following conditions are satisfied: (a) $\mathscr{T}$ is a finite union of $Q$-varieties $\mathscr{T}_{\alpha}$; (b) A subset $Y$ of $\mathscr{T}$ is closed on $\mathscr{T}$ if and only if every $Y_{\alpha}=\mathscr{T}_{\alpha} \cap Y$ is closed on $\mathscr{T}_{\alpha}$ (the topology on $\mathscr{T}_{\alpha}$ is the quotient topology). A field $k$ will be called a field of definition of $\mathscr{T}$ if every $\mathscr{T}_{\alpha}$ is defined over $k$.

When $\mathscr{T}$ and $\mathscr{T}^{\prime}$ are $Q$-spaces, a continuous map $f$ of an open subset of $\mathscr{T}$ into $\mathscr{T}^{\prime}$ will be called a rational map of $\mathscr{T}$ into $\mathscr{T}^{\prime}$ defined over a field $k$, if $k$ is a common field of definition of the component $Q$-varieties of $\mathscr{T}, \mathscr{T}^{\prime}$ and if $f$ induces a rational map $f_{\alpha}$ of a component $Q$-variety $\mathscr{T}_{\alpha}$ of $\mathscr{T}$ defined over $k$ for each $\alpha$. Let $t_{0}$ be a point of $\mathscr{T}$ and $J$ the set of indices $\alpha$ such that $t_{0} \in \mathscr{T}_{\alpha}$. Then we shall say that $f$ is single-valued at $t_{0}$ with the value $t_{0}^{\prime}$ when $t_{0}^{\prime}$ is a unique specialization of $f\left(t_{\alpha}\right)$ over $k$ over the specialization $t_{\alpha} \rightarrow t_{0}$ ref. $k$ of a generic point $t_{\alpha}$ of $\mathscr{T}_{\alpha}$ over $k$ for $\alpha \in J$. $f$ will be called a birational map if $f^{-1}$ is defined and is a rational map of $\mathscr{T}^{\prime}$ into $\mathscr{T}$. A birational map $f$ between $\mathscr{T}$ and $\mathscr{T}^{\prime}$ will be called a one-to-one birational map if $f$ and $f^{-1}$ are everywhere single-valued rational maps.

Proposition 12. Let $\boldsymbol{V}$ be a non-ruled polarized variety of rank at most $d$ and $\Sigma$ an admissible subset of $\Sigma(\boldsymbol{V}, d)$, containing $\boldsymbol{V}$. Let $\mathfrak{\Im}$ be a typical universal family of $\Sigma, F$ the set of Chow-points of members of $F$ and the $F_{\alpha}$ the components of $F$. Let $E$ (resp. $E_{\alpha}$ ) be the set of pairs of equivalent points on $F$ (resp. $F_{\alpha}$ ), $\mathscr{T}$ the abstract quotient space of $F$ by the equivalence relation on it and $f$ the abstract canonical morphism of $F$ on $\mathscr{T}$. Then:

(a) $\left(F_{\alpha}, E_{\alpha}\right)$ is an unmixed equivalence pair and $E_{\alpha}$ is the support of a subvariety of $F_{\alpha} \times F_{\alpha}$; (b) $E_{\alpha}$ is the restriction of $E$ on $F_{\alpha} \times F_{\alpha}$ and $E=\cup_{\alpha} E_{\alpha}$; (c) When $u$ is a point of $F_{\alpha}$, representing $\boldsymbol{U}, E \cap(F \times u)=E_{\alpha} \cap\left(F_{\alpha} \times u\right)=$ $\mathfrak{p}\left(\boldsymbol{U}, \mathfrak{c}_{a}\left(C_{\boldsymbol{U}}\right)\right) \times u$; (d) $E$ is closed on $F \times F ;$ (e) When $f_{\alpha}$ is the restriction of $f$ on $F_{\alpha}$ and $\mathscr{T}_{\alpha}=f_{\alpha}\left(F_{\alpha}\right), \mathscr{T}_{\alpha}$ can be identified with a Q-variety $\left(F_{\alpha}, f_{\alpha}\right)$; (f) When we introduce the quotient topology on $\mathscr{T}, \mathscr{T}$ is a Q-space such that $\mathscr{T}=U_{\alpha} \mathscr{T}_{\alpha}$ and that $f$ is an everywhere single valued rational map of a $Q$-space $F$ onto $T$; (g) When $u^{\prime}$ is a point of $F$ representing $\boldsymbol{U}^{\prime}$ and when $k$ is a common 
field of definition of the $F_{\alpha}, \mathscr{T}_{\alpha}$ and the $f_{\alpha}, k\left(f\left(u^{\prime}\right)\right)$ is the field of moduli of $\boldsymbol{U}^{\prime}$ over $k$.

Proof. Equivalence relations on $F$ and $F_{\alpha}$ are determined by isomorphisms of polarized varieties. Then (c) follows at once from the definitions and from the Corollary to Theorem 5 . Let now $u$ be a generic point of $F_{\alpha}$ over $k$, representing $\boldsymbol{U} . \quad \mathbb{E}_{a}\left(C_{\boldsymbol{U}}\right)^{+}$is a complete total family defined over $k(u)$ by Proposition 2 and Theorem $G$. Hence $\mathfrak{p}\left(\boldsymbol{U}, \mathfrak{E}_{a}\left(\boldsymbol{C}_{\boldsymbol{U}}\right)\right)$ is defined over $k(u)$ by Theorem $E$. There is a subvariety $\widetilde{E}_{\alpha}$ of $F_{\alpha} \times F_{\alpha}$ defined over $k$ and having the projection $F_{\alpha}$ on the first factor, such that $\widetilde{E}_{\alpha} \cdot\left(u \times F_{\alpha}\right)=u \times \widetilde{E}_{\alpha}(u)=u \times \mathfrak{p}\left(\boldsymbol{U}, \widetilde{C}_{a}\left(C_{\boldsymbol{U}}\right)\right)$ (cf. Corollary of Theorem 5; [23], Chap. VIII, Theorem 6). Since $u$ is a point of $\widetilde{E}_{\alpha}(u)$, it follows that $\widetilde{E}_{\alpha}$ has the projection $F_{\alpha}$ on the second factor of the product. When $\left(u^{\prime}, w^{\prime}\right)$ is in $E_{\alpha}$, it follows easily from Proposition 6 that it is in $\widetilde{E}_{\alpha}$. When $\left(u^{\prime}, w^{\prime}\right)$ is in $\widetilde{E}_{\alpha}$, it is in $E_{\alpha}$ since the equivalence relation on $F$ is strongly closed (cf. Theorems 6 and 7). It follows that $\widetilde{E}_{\alpha}=E_{\alpha}$ and (a) follows from these and from the Corollary of Proposition 11.

(b) follows from the definition of our equivalence relation and from the Corollary of Theorem 5. (d) follows from Theorems 6 and 7 . (e) follows from the definition of a $Q$-variety and from what we have proved above. $\mathscr{T}$ is clearly the union of the $\mathscr{T}_{\alpha}$. Let $S$ be a subset of $\mathscr{T}$. When we consider the quotient topology on $\mathscr{T}, S$ is closed on $\mathscr{T}$ if and only if $f^{-1}(S)$ is closed on $F . f^{-1}(S)$ is closed on $F$ if and only if $f^{-1}(S) \cap F_{\alpha}=$ $f^{-1}(S)_{\alpha}$ is closed on $F_{\alpha}$ for each $\alpha$ (cf. Proposition 5.1 of the Appendix). $f^{-1}(S)_{\alpha}$ is stable by the equivalence relation on $F_{\alpha}$ by the Corollary of Theorem 5. Therefore, $f^{-1}(S)_{\alpha}$ is closed on $F_{\alpha}$ if and only if $f_{\alpha}\left(f^{-1}(S)_{\alpha}\right)$ is closed on the $Q$-variety $\mathscr{T}_{\alpha}$ since the topology on $\mathscr{T}_{\alpha}$ is the quotient topology of $F_{\alpha}$ with respect to the equivalence relation. By the definition of $f$ and $f_{\alpha}$ and using the Corollary of Theorem 5, it is easy to verify that $f_{\alpha}\left(f^{-1}(S)_{\alpha}\right)=S \cap \mathscr{T}_{\alpha}$. Therefore, $\mathscr{T}$ is a $Q$-space. Then $f$ is an everywhere single-valued rational map by (e). (g) follows from the definition of a typical universal family, Proposition 2 and from Theorem 2.

Remark 6.1. Our proposition is valid for $\Sigma_{0}(\boldsymbol{V}, d)$ too.

Remark 6.2. In order to define $F, F_{\alpha}$, we have used the concept of Chow-points for the sake of convenience. But this is not essential. For 
instance, the $F_{\alpha}$ may be replaced by those algebraic varieties $F_{\alpha}^{\prime}$ such that (a) $U_{\alpha} F_{\alpha}^{\prime}$ is a locally closed subset of a projective space, that (b) there is a one-to-one birational correspondence between $U_{\alpha} F_{\alpha}$ and $U_{\alpha} F_{\alpha}^{\prime}$ as $Q$ spaces and that (c) when $k$ is a common field of definition of the $F_{\alpha}$, the $F_{\alpha}^{\prime}$ and the birational correspondence $J$, then $k(x)=k(J(x))$ for any point of $U_{\alpha} F_{\alpha}$.

Let $\boldsymbol{V}$ be a non-ruled polarized variety of rank at most $d$ and $\Sigma$ an admissible subset of $\Sigma(\boldsymbol{V}, d)$, containing $\boldsymbol{V}$. Let $\mathscr{T}$ be a $Q$-space with the component $Q$-varieties $\mathscr{T}_{\alpha}$. Suppose that $\mathscr{T}$ satisfies the following conditions:

$\left(M_{1}\right)$ There is an abstract morphism $\beta^{*}$ of $\Sigma$ on $\mathscr{T} \cup \infty$ and $\beta^{*}(\boldsymbol{U}) \neq \infty$ if and only if $\boldsymbol{U}$ is a solid polarized variety of $\Sigma$;

$\left(M_{2}\right)$ When $\boldsymbol{U}$ and $\boldsymbol{U}^{\prime}$ are solid polarized varieties of $\Sigma$, then $\beta^{*}(\boldsymbol{U})=$ $\beta^{*}\left(\boldsymbol{U}^{\prime}\right)$ if and only if $\boldsymbol{U}$ and $\boldsymbol{U}^{\prime}$ are isomorphic;

$\left(M_{3}\right)$ When $\mathfrak{F}$ is a typical universal family of $\Sigma$, the $\mathfrak{F}_{\alpha}$ the components of $\mathfrak{F}$, the $F_{\alpha}$ the Chow-varieties of the $\mathfrak{F}_{\alpha}$ and when $F=\cup_{\alpha} F_{\alpha}, \beta^{*}$ induces a surjective map of $F$ on $\mathscr{T}$, a surjective and everywhere single-valued map $\beta$ of $F$ on $\mathscr{T}$ and $(\mathscr{T}, \beta)$ is the quotient space of the topological space $F$ with respect to the equivalence relation on $F$;

$\left(M_{4}\right) \quad \beta$ induces on $F_{\alpha}$ an everywhere single-valued rational map $\beta_{\alpha}$, mapping $F_{\alpha}$ onto $\mathscr{T}_{\alpha}$ after rearrangement of indices is made if necessary; moreover, there is a common field $k$ of definition of the $\mathscr{T}_{\alpha}$ such that when $u$ is a point of $F_{\alpha}$ representing $\boldsymbol{U}, k(\beta(u))$ is the field of moduli of $\boldsymbol{U}$ over $k$.

When $\mathscr{T}$ satisfies these conditions, we shall say that $\mathscr{T}$ is a $Q$ space of moduli of $\Sigma$ defined over $k$ and $\beta^{*}$ the canonical morphism of $\Sigma$. We shall use similar definitions for admissible subsets of $\Sigma_{0}(\boldsymbol{V}, \boldsymbol{d})$.

TheOREM 8. Let $\boldsymbol{V}$ be a non-ruled polarized variety of rank at most $d$ and $\Sigma$ an admissible subset of $\Sigma(\boldsymbol{V}, d)$, containing $\boldsymbol{V}$. Then it admits a $Q$-space of moduli $\left(\mathscr{T}, \beta^{*}\right)$, which is uniquely determined up to a one-to-one birational correspondence. When there is a typical universal family of $\Sigma$ such that every component of it is defined over a field $k$, there is a $Q$-space of moduli defined over $k$ and vice versa.

Proof. By Theorems 5 and $7, \Sigma$ admits a typical universal family $\mathfrak{F}$. 
Let the $\mathfrak{F}_{\alpha}$ be the components of $\mathfrak{F}$, the $F_{\alpha}$ the Chow-varieties of the $\mathfrak{F}_{\alpha}$, $F=\cup_{\alpha} F_{\alpha}$ and $(\mathscr{T}, \beta)$ as in Proposition 12 relative to $F$ (using $\beta$ for $f$ ). Let $k$ be a common field of definition of the $F_{\alpha}$. The $Q$-variety $\mathscr{T}_{\alpha}=$ $\left(F_{\alpha}, \beta_{\alpha}\right)$ is defined over $k$ by Proposition 2 and by Theorems $E$ and $G$ since $E_{\alpha}$ is a variety defined over $k$ (cf. Proposition 12, (a) and (c)). When $\boldsymbol{U}$ is a solid polarized variety of $\Sigma$, isomorphic to a member $\boldsymbol{U}^{\prime}$ of $\mathfrak{F}$ and $u^{\prime}$ the Chow-point of $\boldsymbol{U}^{\prime}$, we set $\beta^{*}(\boldsymbol{U})=\beta\left(u^{\prime}\right)$. Otherwise, we set $\beta^{*}(\boldsymbol{U})=\infty$.

Let $\mathfrak{F}^{\prime}$ be another typical universal family of $\Sigma$ and $\left(\mathscr{T}^{\prime}, \beta^{\prime *}\right)$ the corresponding pair. There is a map $\psi$ such that $\beta^{\prime *(U)}=\psi\left(\beta^{*}(\boldsymbol{U})\right)$ between $\mathscr{T}$ and $\mathscr{T}^{\prime}$. By the definitions, Proposition 12 and by the Corollary of Proposition 11, $\psi$ is canonically determined and is a one-to-one birational correspondence between $\mathscr{T}$ and $\mathscr{T}^{\prime}$. It follows from this that $\left(\mathscr{T}, \beta^{*}\right)$ is a $Q$-space of moduli of $\Sigma$ defined over $k$ and that it is determined up to a one-to-one birational map.

Let now $\left(\mathscr{T}, \beta^{*}\right)$ be a $Q$-space of moduli defined over a field $k$ and the $\mathscr{T}_{\alpha}$ the component $Q$-varieties of $\mathscr{T}$. Let $\mathscr{F}^{\prime}$ be a typical universal family of $\Sigma, K$ a common field of definition of the components $\mathfrak{F}_{\alpha}^{\prime}$ of $\mathfrak{F}^{\prime}$ over $k$ and $\boldsymbol{U}$ a generic member of $\mathfrak{F}_{\alpha}^{\prime}$ over $K$. Set $\beta^{*}(\boldsymbol{U})=t$. $t$ is a generic point of a component of $\mathscr{T}$ over $K$. Since it is defined over $k, t$ is a generic point of it over $k$. Since $K(t)$ (resp. $k(t)$ ) is the field of moduli of $\boldsymbol{U}$ over $K$ (resp. $k$ ), it is the smallest field of definition of $\mathfrak{p}\left(\boldsymbol{U}, \mathfrak{C}_{a}\left(C_{\boldsymbol{U}}\right)\right)$ over $K$ (resp. $k$ ) by Proposition 2 and Theorem 2. By the Corollary of Theorem 5 and by Theorem 7, it follows easily that $u=\mathfrak{c}(\boldsymbol{U})$ is a generic point of $\mathfrak{p}\left(\boldsymbol{U}, \mathfrak{C}_{a}\left(C_{\boldsymbol{U}}\right)\right)$ over $K(t)$. Then it is so over $k(t)$. From these, it follows at once that $u$ has the same locus over $k$ as over $K$, which is the closure of the Chow-variety of $\mathfrak{F}_{\alpha}^{\prime}$. Let $\bar{F}^{\prime}$ be the union of these. The correspondence $u \rightarrow \beta^{*}(\boldsymbol{U})=t$ is a rational map of the $Q$-space $\bar{F}^{\prime}$ into the $Q$-space $\mathscr{T}$. Since $k(u)$ contains $k(t)$ by Theorems $E, G, 2$ and by Proposition 2 , the rational map is defined over $k$. When $\Gamma$ is the closure of the graph of the rational map on $\bar{F}^{\prime} \times \mathscr{T}$, it is $k$-closed and the set of Chowpoints $F^{\prime}$ of members of $\mathfrak{F}^{\prime}$ is the set-theoretic projection of $\Gamma$ on $\bar{F}^{\prime}$. Hence $\mathfrak{F}^{\prime}$ has to be normally algebraic over $k$, i.e. locally $k$-closed. Our theorem is thereby proved.

Remark 6.3. This theorem is valid for admissible subsets of $\Sigma_{0}(\boldsymbol{V}, d)$ too. 
TheOREm 9. Let $\boldsymbol{V}$ be a non-ruled polarized variety of rank at most $d$ and $\Sigma$ an admissible subset of $\Sigma(\boldsymbol{V}, d)$, containing $\boldsymbol{V}$. Let $\mathfrak{F}_{0}$ be a universal family of $\Sigma$, contained in $\Sigma$ and $\left(\mathscr{T}, \beta^{*}\right)$ the $Q$-space of moduli of $\Sigma$ defined over a field k. Let $F$ be an open subset of the Chow-variety of a component of $\mathfrak{F}_{0}, \mathscr{T}^{\prime}$ a $Q$ space and $\gamma$ an everywhere single-valued rational map of $F$ into $\mathscr{T}^{\prime}$ satisfying the following conditions:

(a) There is a component Q-variety $\mathscr{T}_{\alpha}$ of $\mathscr{T}$ such that $\beta^{*}$ induces on $F$ a generically surjective map $\beta$ to $\mathscr{T}_{\alpha}$; (b) If $u, u^{\prime}$ are points of $F$ which represent isomorphic polarized varieties of $\mathfrak{F}_{0}$, then $r(u)=\gamma\left(u^{\prime}\right)$; (c) When $K$ is a common field of definition of $F, \gamma$ and the components of $\mathscr{T}^{\prime}$ over $k$ and when $u$ is a point of $F$ representing $\boldsymbol{U}, K(\beta(u), \gamma(u))=K\left(\beta^{*}(\boldsymbol{U}), \gamma(u)\right)$ is a separable extension of $K(\beta(u))$ $=K\left(\beta^{*}(\boldsymbol{U})\right)$. Then there is a rational map $h$ of $\mathscr{T}_{\alpha}$ into $\mathscr{T}^{\prime}$ such that $h$ is defined over $K$, single-valued at every $\beta^{*}(\boldsymbol{U})=\beta(u), u \in F$, and that $\gamma=h \circ \beta$ on $F$.

Proof. Clearly there is an abstract morphism $h$ of $\beta(F) \subset \mathscr{T}_{\alpha}$ into $\mathscr{T}^{\prime}$ such that $\gamma=h \circ \beta$ on $F$. By our assumption, when $u$ is a generic point of $F$ over $K, \beta(u)$ is a generic point of $\mathscr{T}_{\alpha}$ over $K$. Let $t^{\prime}$ be a generic specialization of $\beta(u)=t$ over $K(\beta(u))$ and $(t, u) \rightarrow\left(t^{\prime}, u^{\prime}\right)$ an extension of this to a generic specialization of $(t, u)$ over $K(\beta(u))$. Since $K(\beta(u))=$ $K\left(\beta^{*}(\boldsymbol{U})\right)$ is the field of moduli of $\boldsymbol{U}$ over $K, u^{\prime}$ represents a member $\boldsymbol{U}^{\prime}$ of $\mathfrak{\mho}_{0}$ which is isomorphic to $\boldsymbol{U}$. Since $u^{\prime}$ is also in $F$, it follows that $\gamma(u)=$ $r\left(u^{\prime}\right)$. Consequently, $t=t^{\prime}$ and $t$ is rational over $K(\beta(u))$. Our theorem follows at once from this, Theorem 8 and from the definitions.

Remark 6.4. Our theorem is valid for admissible subsets of $\Sigma_{0}(\boldsymbol{V}, d)$ too.

Remark 6.5. The condition (c) is necessary. One can construct an example where $h$ is not a rational map and $K\left(\beta^{*}(\boldsymbol{U}), \gamma(u)\right)$ is a pure inseparable extension of $K\left(\beta^{*}(\boldsymbol{U})\right)$.

The following theorem is an easy exercise of the results we have established and a proof will be left to the reader.

TheOREM 10. The notations and assumptions being those of Theorem 8, there is the smallest normal extension $k$ of the basic field $k_{0}$ of deformations, which has the following properties: (a) There is a Q-space of moduli $\left(T, \beta^{*}\right)$ defined over $k$; 
(b) When $\sigma$ is an automorphism of $k$ over $k_{0}$ and when $\mathscr{T}_{\alpha}$ is a component of $\mathscr{T}, \mathscr{T}_{\alpha}^{\sigma}$ is also a component of $\mathscr{T}$.

\$7. An Example. Let $\boldsymbol{V}^{n}$ be a polarized hypersurface. By this we mean that $\boldsymbol{V}$ is isomorphic to a hypersurface of the projective space $\boldsymbol{P}^{n+1}$. In the case of characteristic 0 , we have $l(\boldsymbol{V}, d)=1$ and $n(\boldsymbol{V}, d)={ }_{n+1+m} C_{m}-(n+2)^{2}$ if $n \geqslant 2, m \geqslant 3$, excluding the case $n=2, h=4$ where $m$ is the degree of the hypersurface in $\boldsymbol{P}^{n+1}$, isomorphic to $\boldsymbol{V}$ (cf. [9]). In the case of characteristic $p$, the same is true if we take for $d$ the rank of $\boldsymbol{V}$ and assume that $\boldsymbol{V}$ is isomorphic to a generic hypersurface over $k_{0}$ in $\boldsymbol{P}^{n+1}$.

Let $\boldsymbol{V}^{n}$ be a polarized variety and set $n=N-s$. Suppose that there is a non-degenerate projective embedding $\boldsymbol{f}$ of $\boldsymbol{V}$ into $\boldsymbol{P}^{N}$ such that $\boldsymbol{f}(\boldsymbol{V})$ is the intersection-product of $s$ properly intersecting hypersurfaces of degrees $m_{i}$. Arrange the $m_{i}$ so that $m_{i-1} \leqslant m_{i}$. Then we shall say that $\boldsymbol{V}^{n}$ is a complete intersection of type $\left(m_{1}, \ldots, m_{s}\right)$. The following facts are well-known (cf. [20]): Let $U$ be the intersection-product of $s$ properly intersecting hypersurfaces $H_{m_{i}}$ of degrees $m_{i}$ in $\boldsymbol{P}^{N}$, then $H^{i}\left(U, \mathfrak{D}_{U}(m)\right)=0$ for $1 \leqslant i \leqslant N-s-1$ and $H^{N-s}\left(U, \mathfrak{D}_{U}(m)\right) \cong H^{0}\left(U, \mathfrak{D}_{U}(\rho-m)\right)^{*}$ where $\rho=\sum m_{i}-N-1, m$ is an arbitrary integer and $*$ indicates the dual.

It is well-known that a non-singular generic hypersurface has the irregularity 0, i.e. its Picard variety is of dimension 0. Also a generic hypersurface section of a non-singular subvariety of dimension $n>2$ of a projective space has the same irregularity as the ambient variety (cf. Matsusaka, "On the Theorem of Castelnuovo-Enriques", Nat. Sci. Rep., Ochanomizu Univ., 1953). When that is so, a complete intersection $\boldsymbol{V}^{n}$ of type $\left(m_{1}, \ldots, m_{s}\right)$ has the irregularity 0 by Theorem $B$ if $n \geqslant 2$. Consequently, algebraic equivalence and linear equivalence on $\boldsymbol{V}$ coincide.

Throughout this paragraph, we shall fix the following notations. $\boldsymbol{V}^{n}$ will denote a complete intersection of type $\left(m_{1}, \ldots, m_{s}\right) . d$ will denote the rank of $\boldsymbol{V}$. We assume that $\boldsymbol{V}$ is isomorphic to the intersection-product $\mathrm{Hm}_{1} \cdots \mathrm{Hm}_{\mathrm{s}}$ of independent generic hypersurfaces over $k_{0}$ in $\boldsymbol{P}^{N}$. This assumption is not necessary when the characteristic is 0 . Furthermore, we set $\left(m_{1}, \ldots, m_{s}\right)=$ $\left(\left(m_{1}, \ldots, m_{1}\right), \ldots,\left(m_{i}, \ldots, m_{i}\right), \ldots,\left(m_{l}, \ldots, m_{l}\right)\right)$, where $m_{i} \neq m_{j}$ if $i \neq j$ and the $m_{i}$ are repeated $t_{i}$-times. We shall be mainly interested in the spaces $\Sigma_{0}^{\prime \prime}(\boldsymbol{V}, d)$ and $\Sigma_{0}^{\prime}(\boldsymbol{V}, d)$. Our immediate goal is to show that every member of $\Sigma_{0}^{\prime \prime}(\boldsymbol{V}, d)$ is also a complete intersection of the same type as $\boldsymbol{V}$. 
Essentially, this corresponds to Theorems 14.1 and 18.5 of [9] within the frame work of abstract algebraic varieties, when $\boldsymbol{V}$ is a hypersurface. As a matter of fact, we can conclude easily from this that the set of nonsingular subvarieties of dimension $n$, degree $\Pi_{1}^{s} m_{i}$ in $\boldsymbol{P}^{N}$, which are specializations of the intersection-product of $s$ independent generic hypersurfaces of degrees $m_{i}$ over $k_{0}$, is a universal family of $\Sigma_{0}^{\prime \prime}(\boldsymbol{V}, d)$, regarding it as a subset of $\Sigma(\boldsymbol{V}, d)$.

Since $\boldsymbol{V}$ is a complete intersection, there is a non-degenerate projective embedding $\boldsymbol{f}$ of $\boldsymbol{V}$ such that $\boldsymbol{f}(\boldsymbol{V})$ can be expressed as the intersectionproduct of $s$ properly intersecting hypersurfaces. Denote by $C_{r}^{*}$ a hypersurface section of $\boldsymbol{f}(\boldsymbol{V})$ by a hypersurface of degree $r$ and set $\boldsymbol{f}^{-1}\left(C_{r}^{*}\right)=$ $C_{r}$. Let $\boldsymbol{W}$ be a member of $\Sigma_{0}^{\prime \prime}(\boldsymbol{V}, d)$ such that $\boldsymbol{V}$ is a specialization of $\boldsymbol{W}$ over a discrete valuation-ring 5 . There is a polar divisor $D_{r}$ on $\boldsymbol{W}$ such that $\left(\boldsymbol{W}, D_{r}\right) \rightarrow\left(\boldsymbol{V}, C_{r}^{\prime}\right)$ ref. 5 , where $C_{r} \sim C_{r}^{\prime}$. We have the following relations: $h^{i}\left(\boldsymbol{W}, D_{r}\right)=h^{i}\left(\boldsymbol{V}, C_{r}\right)=0$ for $0<i<n=N-s$;

$\chi\left(\boldsymbol{W}, D_{r}\right)=\chi\left(\boldsymbol{V}, C_{r}\right)=c_{r} \quad\left(\mathrm{cf}\right.$. Theorem A); $h^{0}\left(\boldsymbol{V}, C_{r}\right)_{-}^{-}=c_{r}-(-1)^{n} h^{n}\left(\boldsymbol{V}, C_{r}\right)$, $h^{0}\left(\boldsymbol{W}, D_{r}\right)=c_{r}-(-1)^{n} h^{n}\left(\boldsymbol{W}, D_{r}\right) ; h^{n}\left(\boldsymbol{W}, D_{r}\right) \leqslant h^{n}\left(\boldsymbol{V}, C_{r}\right)$.

Lemma 6. When $n$ is even, $h^{0}\left(\boldsymbol{V}, C_{r}\right)=h^{0}\left(\boldsymbol{W}, D_{r}\right)$ and $h^{n}\left(\boldsymbol{V}, C_{r}\right)=$ $h^{n}\left(\boldsymbol{W}, D_{r}\right)$.

Proof. If $n$ is even, $h^{0}\left(\boldsymbol{V}, C_{r}\right)=c_{r}-h^{n}\left(\boldsymbol{V}, C_{r}\right)$ and $h^{0}\left(\boldsymbol{W}, D_{r}\right)=c_{r}-h^{n}\left(\boldsymbol{W}, D_{r}\right)$. By the upper semi-continuity, we have $h^{0}\left(\boldsymbol{W}, D_{r}\right) \leqslant h^{0}\left(\boldsymbol{V}, C_{r}\right)$. Hence $h^{n}\left(\boldsymbol{W}, D_{r}\right) \geqslant h^{n}\left(\boldsymbol{V}, C_{r}\right)$. Our lemma follows at once from this.

Lemma 7. Assume that $h^{0}\left(\boldsymbol{V}, C_{r}\right)=h^{0}\left(\boldsymbol{W}, D_{r}\right)$ for all positive integers $r$. Then $\boldsymbol{W}$ is a complete intersection of the same type as $\boldsymbol{V}$.

Proof. $D_{r}$ is ample by Proposition 2.2 of the Appendix. By Proposition 6 , there is a non-degenerate projective embedding $\boldsymbol{g}$ determined by $D_{1}$ such that $\left(\boldsymbol{W}, \Gamma_{\boldsymbol{g}}\right) \rightarrow\left(\boldsymbol{V}, \Gamma_{\boldsymbol{f}}\right)$ ref. 5. Identifying $\boldsymbol{V}, \boldsymbol{W}$ with $\boldsymbol{f}(\boldsymbol{V}), \boldsymbol{g}(\boldsymbol{W})$, we may assume that $\boldsymbol{V}, \boldsymbol{W}$ are already in the projective space of dimension $N$. Let $\Lambda\left(\boldsymbol{V}, m_{i}\right)$ be the linear system of hypersurfaces of degree $m_{i}$, consisting of those hypersurfaces which contain $\boldsymbol{V}$. We have $\operatorname{dim} \Lambda\left(C_{m_{i}}\right)=$ $\operatorname{dim} \Lambda\left(m_{i} H\right)-\operatorname{dim} \Lambda\left(\boldsymbol{V}, m_{i}\right)$ and a similar formula for $\boldsymbol{W}$, where $H$ denotes a hyperplane. Then we get $\operatorname{dim} \Lambda\left(\boldsymbol{V}, m_{i}\right)=\operatorname{dim} \Lambda\left(\boldsymbol{W}, m_{i}\right)$ from our assumption.

Denote by $Z_{i j}, 1 \leqslant i \leqslant l, 1 \leqslant j \leqslant t_{i}$, independent generic members of the $\Lambda\left(\boldsymbol{V}, m_{i}\right), 1 \leqslant i \leqslant l$, over some field of definition of $\boldsymbol{V}$. Then the $Z_{i j}$ 
intersect properly on the ambient projective space and their intersectionproduct is precisely $\boldsymbol{V}$. Denote by $G_{i}, G_{i}^{\prime}$ the supports of the Chow-varieties of the $\Lambda\left(\boldsymbol{W}, m_{i}\right), \Lambda\left(\boldsymbol{V}, m_{i}\right)$, we have $\left(\boldsymbol{W},\left(G_{i}\right)\right) \rightarrow\left(\boldsymbol{V},\left(G_{i}^{\prime}\right)\right)$ ref. $\mathfrak{D}$. Therefore, there are members $T_{i j}$ of $\Lambda\left(\boldsymbol{W}, m_{i}\right)$ such that $\left(\boldsymbol{W},\left(T_{i j}\right)\right) \rightarrow\left(\boldsymbol{V},\left(Z_{i j}\right)\right)$ ref. 5 . The $T_{i j}$ intersect properly, contains $\boldsymbol{W}$, and their intersection-product specializes to $\boldsymbol{V}$ over $\subseteq$ by the compatibility of specializations with intersection-product. Clearly, $\boldsymbol{V}$ and $\boldsymbol{W}$ have the same degree as positive cycles and this proves that $\boldsymbol{W}$ is the intersection-product of the $T_{i j}$. Our lemma is proved.

Proposition 13 ${ }^{3)}$. Let $\boldsymbol{V}$ and $\boldsymbol{W}$ be polarized varieties of rank $d$ such that $\boldsymbol{V}$ is a specialization of $\boldsymbol{W}$ over a discrete valuation-ring $D$. If $\operatorname{dim} \boldsymbol{V}=n>1$ and $\boldsymbol{V}$ is a complete intersection of type $\left(m_{1}, \ldots, m_{s}\right), \boldsymbol{W}$ is a complete intersection of the same type as $\boldsymbol{V}$. There is a non-degenerate projective embedding $\boldsymbol{h}$ (resp. $\boldsymbol{g}$ ) of $\boldsymbol{V}($ resp. $\boldsymbol{W})$ such that $\boldsymbol{h}(\boldsymbol{V})=\boldsymbol{V}^{\prime}$ (resp. $\boldsymbol{g}(\boldsymbol{W})=\boldsymbol{W}^{\prime}$ ) is the intersection-product of $s$ properly intersecting hypersurfaces of the degrees $m_{i}$ and that $\boldsymbol{W}^{\prime} \rightarrow \boldsymbol{V}^{\prime}$ ref. 5 . Moreover, $h^{0}\left(\boldsymbol{V}^{\prime}, r C_{\boldsymbol{V}^{\prime}}\right)=h^{0}\left(\boldsymbol{W}^{\prime}, r C_{\boldsymbol{W}^{\prime}}\right)$ for all integers $r$.

Proof. When $n$ is even, our proposition follows from Lemmas 6 and 7 . Therefore, we assume that $n$ is odd and that $n>2$. Using the same notations as in Lemmas 6 and 7, take $r$ so large that $D_{r}$ and $C_{r}$ are both sufficiently ample. When we denote by $G_{r}, G_{r}^{\prime}$ the supports of the Chowvarieties of $\Lambda\left(D_{r}\right), \Lambda\left(C_{r}\right)$, we have $\left(\boldsymbol{W}, D_{r}, G_{r}\right) \rightarrow\left(\boldsymbol{V}, C_{r}^{\prime}, G_{r}^{\prime}\right)$ ref. $\mathfrak{D}, C_{r} \sim C_{r}^{\prime}$, since $l\left(D_{r}\right)=l\left(C_{r}\right)$ by Proposition 3. Then, when $x^{\prime}$ is a given point of $G_{r}^{\prime}$, there is a point of $G_{r}$ which specializes to $x^{\prime}$ over the above specialization with reference to 5 . Therefore, we may assume, without loss of generality, that $\left(\boldsymbol{W}, D_{r}\right) \rightarrow\left(\boldsymbol{V}, C_{r}\right)$ ref. $\subseteq$ and that $D_{r}, C_{r}$ are non-singular varieties. Since $C_{r}=\boldsymbol{f}^{-1}\left(C_{r}^{*}\right)$ and since $h^{1}\left(U, \mathfrak{D}_{U}(m)\right)=0$ for any non-singular intersection-product of dimension at least 2 of properly intersecting hypersurfaces, the set of hyperplane sections of $C_{r}^{*}$ is complete. This implies that $C_{r}$, together with the induced polarization, is a complete intersection of type $\left(m_{1}, \ldots, m_{s}, r\right)$. Then $D_{r}$ is a complete intersection of the same type by Lemma 7 .

$$
h^{n}\left(\boldsymbol{V}, C_{m}^{\prime}\right)=h^{n-1}\left(C_{r},\left(C_{r}^{\prime}+C_{m}^{\prime}\right) \cdot C_{r}\right) \text { and } h^{n}\left(\boldsymbol{W}, D_{m}^{\prime}\right)=h^{n-1}\left(D_{r},\left(D_{r}^{\prime}+D_{m}^{\prime}\right) \cdot D_{r}\right)
$$
for large $r$, where $C_{r}^{\prime} \sim C_{r}, D_{r}^{\prime} \sim D_{r}, C_{m}^{\prime} \sim C_{m}, D_{m}^{\prime} \sim D_{m}$ and $C_{r}^{\prime}, D_{r}^{\prime}, C_{m}^{\prime}, D_{m}^{\prime}$

3) This proposition does not require our basic assumption that $\boldsymbol{V}$ is isomorphic to the intersection-product of $s$ independent generic hypersurfaces over $k_{0}$. 
are chosen so that the intersections involved are proper. $h^{0}\left(\boldsymbol{V}, C_{m}\right)=h^{0}\left(\boldsymbol{W}, D_{m}\right)$ follows from Lemma $6, h^{0}\left(\boldsymbol{V}, C_{m}\right)=c_{m}-(-1)^{n} h^{n}\left(\boldsymbol{V}, C_{m}\right)$ and the similar formula for $\boldsymbol{W}$ and $D_{m}$. The rest of our proposition now follows from Proposition 6 and Lemma 7.

Let us denote by $\mathfrak{F}\left(N, n ; m_{1}, \ldots, m_{s}\right)$ the set of non-singular varieties in the projective space $\boldsymbol{P}^{N}$ of dimension $n=N-s$ which are specializations of a generic complete intersection of the same type as $\boldsymbol{V}$. We identify it with an algebraic family of polarized varieties of rank $d$, having the same polarization type as $\boldsymbol{V}$. Then, as we have remarked, Proposition 13 and the definitions imply that $\mathfrak{F}\left(N, n ; m_{1}, \ldots, m_{s}\right)$ is a universal family of $\Sigma_{0}^{\prime \prime}(\boldsymbol{V}, d)$ and $l(\boldsymbol{V}, d)=1$. Next, we shall compute the number $n(\boldsymbol{V}, d)$.

Lemma 8. Let $\boldsymbol{W}$ be a member of $\mathfrak{\Im}\left(N, n ; m_{1}, \ldots, m_{s}\right)$ and a complete intersection of type $\left(m_{1}, \ldots, m_{s}\right)$. Let $\Lambda\left(m_{i}\right)$ be the complete linear system of hypersurfaces of degree $m_{i}$ in $\boldsymbol{P}^{N}$ and $\Lambda\left(\boldsymbol{W}, m_{i}\right)$ the linear subsystem of it, consisting of those hypersurfaces which contain $\boldsymbol{W}$. Then the smallest field of definition of $\boldsymbol{W}$ and the smallest field over which the Chow-varieties of the $\Lambda\left(\boldsymbol{W}, m_{\imath}\right)$ are defined coincide.

Proof. Let $K$ be the smallest field of definition of $\boldsymbol{W}$ and $L$ the smallest common field of definition of the Chow-varieties of the $\Lambda\left(\boldsymbol{W}, m_{i}\right)$. First we shall show that $L$ contains $K$. There are members $H_{m_{i}}$ of the $\Lambda\left(\boldsymbol{W}, m_{i}\right)$ such that they intersect properly and that $\boldsymbol{W}=H_{m_{1}} \cdots H_{m_{s}}$. Hence, we may assume that these $H_{m_{i}}$ are independent generic members of the linear systems over $L$. Let $L^{\prime}$ be a common field of rationality of the $H_{m_{\imath}}$ over $L$. Then $\boldsymbol{W}$ is rational and hence defined over $L^{\prime}$. We may take $L^{\prime}$ to be regular over $L$. Taking an independent generic specialization of the $H_{m_{i}}$ over $L$, we see that $\boldsymbol{W}$ is also defined over a regular extension $L^{\prime \prime}$ of $L$ which is independent over $L$ with respect to $L^{\prime}$. Hence $\boldsymbol{W}$ is defined over $L$ and $L$ contains $K$.

Next we shall show that $K$ contains $L$. For this it is enough to know that the module of forms of degree $m$ which vanish on $\boldsymbol{W}$ has a basis over $K$. But this is an easy exercise. Hence we have our lemma.

Lemma 9. Let $\operatorname{dim} \Lambda\left(m_{i}\right)=v_{i}$ and $\operatorname{dim} \Lambda\left(\boldsymbol{V}_{0}, m_{i}\right)=u_{i}$ where $\boldsymbol{V}_{0}$ is a member of $\mathfrak{F}\left(N, n ; m_{1}, \ldots, m_{s}\right)$ and is a complete intersection of type $\left(m_{1}, \ldots, m_{s}\right)$. Then $\operatorname{dim} \mathfrak{F}\left(N, n ; m_{1}, \ldots, m_{s}\right)=\sum v_{i}-\sum u_{i}$.

Proof. Let the $H_{m_{i}}$ be independent generic members of the $\Lambda\left(m_{i}\right)$ over 
a field of definition $k$ of $\mathfrak{F}\left(N, n ; m_{1}, \ldots, m_{s}\right)$. Then clearly the ntersectionproduct $\boldsymbol{W}$ of the $H_{m_{i}}$ is a generic member of this universal family over $k$. Hence $\boldsymbol{V}_{0}$ is a specialization of $\boldsymbol{W}$ over $k$ in particular. By Proposition 13, we have $h^{0}\left(\boldsymbol{V}_{0}, \boldsymbol{V}_{0} \cdot H_{m_{i}}\right)=h^{0}\left(\boldsymbol{W}, \boldsymbol{W} \cdot H_{m_{i}}\right)$. Hence the formula $h^{0}\left(\boldsymbol{W}, \boldsymbol{W} \cdot H_{m_{i}}\right)-1$ $=\operatorname{dim} \Lambda\left(m_{i}\right)-\operatorname{dim} \Lambda\left(\boldsymbol{W}, m_{i}\right)$ and the similar formula for $\boldsymbol{V}_{0}$ show that $\operatorname{dim} \Lambda\left(\boldsymbol{V}_{0}, m_{i}\right)=\operatorname{dim} \Lambda\left(\boldsymbol{W}, m_{i}\right)=u_{i}$.

Let the $G_{i}$ be the Chow-varieties of the $\Lambda\left(m_{i}\right)$ and $F$ the Chow-variety of $\mathfrak{F}\left(N, n ; m_{1}, \ldots, m_{s}\right)$. Set $G=\Pi_{i} G_{i}$. Let the $x_{i}$ be the Chow-points of the $H_{m_{i}}$ and $w$ the Chow-point of $\boldsymbol{W}$. Denote by $T$ the locus of $\left(x_{1}, \ldots, x_{s}, w\right)$ over $\bar{k}$. Since $k\left(x_{1}, \ldots, x_{s}\right)$ is regular over $k$ and since $w$ is rational over $k\left(x_{1}, \ldots, x_{s}\right), T$ is defined over $k$ and is the closure of the graph of a rational map of $G$ into $F$. The projection of $T$ on $F$ is $F$, and the cycle $T^{-1}(w)$ which is defined by $(G \times w) \cdot T=T^{-1}(w) \times w$ is prime rational over $k(w)$. From the definition of $T$ and from Lemma 8 , it is then easy to see that $\left|T^{-1}(w)\right|$ is the product of the supports of the Chowvarieties of the $\Lambda\left(\boldsymbol{W}, m_{i}\right)$. Our lemma is an immediate consequence of these results.

Corollary. $\operatorname{dim} \mathfrak{F}\left(N, n ; m_{1}, \ldots, m_{s}\right)=\sum_{i} h^{0}\left(\boldsymbol{V}_{0}, \boldsymbol{V}_{0} \cdot H_{m_{i}}\right)-s$

Proposition 14. Let $\boldsymbol{W}$ be a generic member of the universal family $\mathfrak{F}\left(N, n ; m_{1}, \ldots, m_{s}\right)$ of $\Sigma_{0}^{\prime \prime}(\boldsymbol{V}, d)$ over a field $k$ of definition of it. Then $n(\boldsymbol{V}, d)$ $=\sum_{i} h^{0}\left(\boldsymbol{U}, \boldsymbol{U} \cdot H_{m_{i}}\right)-s-(\operatorname{dim} P G L(N)-\operatorname{dim}$ aut $(\boldsymbol{W}))$, where $\boldsymbol{U}$ denotes an arbitrary member of the universal family.

Proof. Our formula is an immediate consequence of the definitions and of Lemma 9, Corollary of Theorem $F$.

Proposition 15. The set $\mathfrak{S}\left(N, n ; m_{1}, \ldots, m_{s}\right)$ of members of $\mathfrak{F}(N, n$; $\left.m_{1}, \ldots, m_{s}\right)$ which are complete intersections of type $\left(m_{1}, \ldots, m_{s}\right)$ is an irreducible algebraic family such that its Chow-variety is an open subset of that of $\mathfrak{F}\left(N, n ; m_{1}, \ldots, m_{s}\right)$ over $k_{0}$. When $\rho=\sum m_{i}-N-1 \geqslant 0$, every member of $\mathfrak{F}\left(N, n ; m_{1}, \ldots, m_{s}\right)$ is not ruled, and, for such a member $\boldsymbol{U}$, we have $\operatorname{dim} \operatorname{aut}(\boldsymbol{U})=0$.

Proof. The first assertion in our proposition is an easy exercise.

As we remarked, every member of $\mathfrak{F}\left(N, n ; m_{1}, \ldots, m_{s}\right)$ is a regular variety by Theorem $B$. For the sake of simplicity, let us identify a member 
$W$ of $\mathfrak{E}\left(N, n ; m_{1}, \ldots, m_{s}\right)$ with its underlying variety $W$. A canonical divisor of $\boldsymbol{P}^{N}$ is a hypersurface of degree $-N-1$ as is well-known and easy to prove. In general, when $H_{m_{1}}, \ldots, H_{m_{s}}$ are properly intersecting hypersurfaces such that their intersection-product is non-singular, a canonical divisor of $H_{m_{1}} \cdots H_{m_{s}}$ is given by $H_{m_{1}} \cdots H_{m_{s}} \cdot H_{\rho}$, where $\rho=\sum m_{i}-N-1$ (cf. [27]). Therefore, when $\rho$ is positive, a canonical divisor of $W$ is an ample divisor. Since the underlying variety $U$ of a member of $\mathfrak{F}\left(N, n ; m_{1}, \ldots, m_{s}\right)$ is a specialization of some $W$ over $k_{0}$, it follows that $U \cdot H_{\rho}$ is also a canonical divisor on $U$ and that a canonical divisor on $U$ is either ample or linearly equivalent to 0 . The connected component $G$ of aut $(U)$ leaves every divisor class on $U$ with respect to algebraic equivalence fixed. Moreover, the irregularity of $U$ is 0 . Hence $G$ leaves every complete linear system of divisors on $U$ invariant. Consequently, $G$ has a non-trivial representation as a linear algebraic group.

If $U$ is a ruled variety, it is birationally equivalent to $\boldsymbol{P}^{1} \times D^{n-1}$ where $D^{n-1}$ is a normal projective variety. A canonical divisor on this product is linearly equivalent to $K\left(\boldsymbol{P}^{1}\right) \times D+\boldsymbol{P}^{1} \times K\left(D^{n-1}\right)$, where $K(*)$ denotes a canonical divisor of $*$. This is well-known and easy to verify. Then it is easy to see that the virtual geometric genus $p_{g}\left(\boldsymbol{P}^{1} \times D^{n-1}\right)$ is 0 , and, when that is so, the geometric genus $p_{g}(U)$ has to be 0 (cf. [27]). Therefore, $U$ cannot be a ruled variety.

If $U$ cannot be a ruled variety, the connected component of aut $(U)$ containing the identity is an Abelian variety (cf. [16]). Hence it has to be reduced to the identity as is well-known since it has a non-trivial representation as a linear algebraic group (cf. M.Rosenlicht, "Some rationality questions on algebraic groups", Annali di Matematica, 1957; H. Matsumura, "On algebraic groups of birational transformations", Rendiconti, Accad. Naz. Lincei, 34, 1963). Our proposition is thereby proved.

Proposition 16. There is a non-singular algebraic variety $A$ and a subvariety $Z$ of $A \times \boldsymbol{P}^{N}$ with the following properties: (a) $x \times \boldsymbol{P}^{N}$ and $Z$ intersect properly on $A \times \boldsymbol{P}^{N}$ for every point $x$ of $A$; (b) $Z(x)$ which is defined by $(x \times \boldsymbol{P}) \cdot Z=$ $x \times Z(x)$ is a member of $\mathfrak{S}\left(N, n ; m_{1}, \ldots, m_{\mathbf{s}}\right)$ when polarized suitably, and vice versa; (c) $A$ and $Z$ are defined over the basic field $k_{0}$ and $k_{0}(x)$ is the smallest field of definition of $Z(x)$ over $k_{0}$.

Proof. When $A$ is a linear system of dimension $r$ of divisors on a complete variety $B$ without singular subvariety of co-dimension 1 , there is 
a positive cycle $T$ on $\boldsymbol{P}^{r} \times B$ such that (i) $y \times B$ and $T$ intersect properly on $\boldsymbol{P}^{r} \times B$ for every $y$ on $\boldsymbol{P}^{r}$, (ii) $T(y)$ is in $\Lambda$ and conversely, and that (iii) when $k$ is a common field of definition of $B$ and $A, k(y)$ is the smallest field of rationality of $T(y)$ containing $k$. This fact is well-known and we shall call it a Grassman parametrization of $\Lambda$ (cf. [23], Chap. IX).

Denote by $\mathfrak{S}$ the family $\mathfrak{S}\left(N, n ; m_{1}, \ldots, m_{s}\right)$ and let $\boldsymbol{U}$ be a member of S. Set $\operatorname{dim} \Lambda\left(m_{i}\right)=v_{i}, \quad \boldsymbol{P}_{i}=\boldsymbol{P}^{v_{i}}, \boldsymbol{P}^{\prime}=\Pi_{i} \boldsymbol{P}_{i} \quad$ and $\operatorname{dim} \Lambda\left(\boldsymbol{U}, m_{i}\right)=u_{i} . \quad u_{i}$ is independent of the choice of $\boldsymbol{U}$ by Proposition 13. Denote by $\left(\boldsymbol{P}_{i}, T_{i}\right)$ the Grassman parametrization of $\Lambda\left(m_{i}\right)$ and $\left(x^{\prime}\right)=\left(x_{1}, \ldots, x_{s}\right)$ a point of $\boldsymbol{P}^{\prime}$ such that $x_{i}$ is a point of $\boldsymbol{P}_{i}$. For the sake of simplicity, we shall identify members of $\mathfrak{H}$ with their underlying varieties.

Let $\boldsymbol{P}_{0}^{\prime}$ be the set of points $(x)$ of $\boldsymbol{P}^{\prime}$, such that the $T_{i}\left(x_{i}\right)$ intersect properly on $\boldsymbol{P}^{N}$ and that $T_{1}\left(x_{1}\right) \cdots T_{s}\left(x_{s}\right)$ is non-singular, forms an open subset of $\boldsymbol{P}^{\prime}$ which is easy to prove. When $(x)$ is in $\boldsymbol{P}_{0}^{\prime}, T_{1}\left(x_{1}\right) \cdots T_{s}\left(x_{s}\right)=$ $U(x)$ is a member of $\mathfrak{S}$, and conversely, a member $U$ of $\mathfrak{S}$ can be expressed this way. For a point $(x)$ of $\boldsymbol{P}_{0}^{\prime}$, the linear system $\Lambda\left(U(x), m_{i}\right)$ is represented by a subspace $L\left((x), m_{i}\right)$ of dimension $u_{i}$ in $\boldsymbol{P}_{i}$. Set $L((x))=\Pi_{i} L\left((x), m_{i}\right)$. We shall show that the $L((x))$ form an involutional family of positive cycles on $\boldsymbol{P}^{\prime}$, then apply a theorem of Chow $((2))$ to construct $A$ and $Z$.

In order to do so, we first observe the following basic properties are satisfied by the $L((x))$. (i) For $(x) \in \boldsymbol{P}_{0}^{\prime}, L((x))$ is uniquely determined, $\operatorname{dim} L((x))=\sum u_{i}$ and $U(x)$ and $L((x))$ have the same smallest common field of definition over $k_{0}$ (cf. Lemma 8). (ii) When $(x),\left(x^{\prime}\right) \in \boldsymbol{P}_{0}^{\prime}$ and $(x) \rightarrow\left(x^{\prime}\right)$ ref. $k_{0}, L((x)) \rightarrow L\left(\left(x^{\prime}\right)\right)$ ref. $k_{0}$ uniquely over this specialization. (iii) When $(x),\left(x^{\prime}\right) \in \boldsymbol{P}_{0}^{\prime}$ such that $\left(x^{\prime}\right) \in L((x))$, then $U(x)=U\left(x^{\prime}\right)$ and vice versa. (iv) When $(x),\left(x^{\prime}\right) \in \boldsymbol{P}_{0}^{\prime}$ and $L((x)) \rightarrow L\left(\left(x^{\prime}\right)\right)$ ref. $k_{0}$, then $U(x) \rightarrow U\left(x^{\prime}\right)$ ref. $k_{0}$ uniquely over this specialization.

(i) and (ii) show that the $L((x))$ form generically an involutional system on $\boldsymbol{P}^{\prime}$ in the sense of Chow (cf. [2]). Let $A$ be the set of Chow-points of the $L((x))$ when $(x)$ is in $\boldsymbol{P}_{0}^{\prime}$. Then every point of $A$ is simple on its closure $\bar{A}$ by the theorem of Chow. $A$ is the image of $\boldsymbol{P}_{0}^{\prime}$ by the canonical rational map of $\boldsymbol{P}^{\prime}$ into $\bar{A}$, and the map is defined at every point of $\boldsymbol{P}_{0}^{\prime}$. Since $A$ contains always a non-empty open subset of $\bar{A}$ (cf. [24]), it is easy to prove that $A$ is actually open on $\bar{A}$. Then (i)-(iv) prove the existence of $Z$ as required in our proposition, which is an easy exercise of [23], 
Theorem 6, Chap. VIII and of the compatibility of specializations with intersection-product.

Proposition 17. Call $\mathfrak{S}_{d}\left(N, n ; m_{1}, \ldots, m_{s}\right)$ the subset of $\mathfrak{S}\left(N, n ; m_{1}, \ldots, m_{s}\right)$, consisting of polarized varieties of rank $d$. Then $\mathfrak{H}_{d}\left(N, n ; m_{1}, \ldots, m_{s}\right)$ is an irreducible algebraic family defined over $k_{0}$. Moreover, Proposition 16 is valid for $\mathfrak{H}_{d}\left(N, n ; m_{1}, \ldots, m_{s}\right)$ too if we take a suitable open subset of $A$ which is defined over $k_{0}$.

Proof. This follows at once from Proposition 16 and from Proposition 4. 3 of the Appendix.

Let us denote by $\Sigma^{*}$ the subset of $\Sigma_{0}^{\prime}(\boldsymbol{V}, d)$ consisting of complete intersections of type $\left(m_{1}, \ldots, m_{s}\right)$. Then Propositions 15 and 17 show that $\Sigma^{*}$ is an admissible subset of $\Sigma_{0}(\boldsymbol{V}, d)$, that $\mathfrak{H}_{d}\left(N, n ; m_{1}, \ldots, m_{s}\right)$ is a universal family of $\Sigma^{*}$ contained in it and that an arbitrary member of $\Sigma_{0}^{\prime}(\boldsymbol{V}, d)$ can be obtained by specialization over $k_{0}$ from a member of $\Sigma^{*}$ (cf. Proposition 13).

Theorem 11. Let $\boldsymbol{V}^{n}$ be a polarized variety of rank $d$. Assume that it is a complete intersection of type $\left(m_{1}, \ldots, m_{s}\right)$ and isomorphic to the intersection-product of $s$ independent generic hypersurfaces $H_{m_{i}}$ of degrees $m_{i}$ over $k_{0}$ in $\boldsymbol{P}^{N}$. Set $U=$ $H_{m_{i}} \cdots H_{m_{s}}$. Then we have the following results (assume that $m_{1}>1$ and $n>1$ ): (a) $l(\boldsymbol{V}, d)=1, n(\boldsymbol{V}, d)=\sum_{i} h^{0}\left(U, 5_{U}\left(m_{i}\right)\right)-s-(\operatorname{dim} P G L(N)-t)$ where $t=$ $\operatorname{dim}$ aut $(\boldsymbol{V})$;

(b) When $\rho=\Sigma m_{i}-N-1 \geqslant 0$, every member of $\mathfrak{S}\left(N, n ; m_{1}, \ldots, m_{s}\right)$ is not ruled and $\operatorname{dim}$ aut $(\boldsymbol{V})=0$;

(c) When $\Sigma^{*}$ is the set of members of $\Sigma_{0}^{\prime}(\boldsymbol{V}, d)$ which are complete intersections of type $\left(m_{1}, \ldots, m_{s}\right)$, it is an admissible subset of $\Sigma_{0}(\boldsymbol{V}, d)$;

(d) When $\rho \geqslant 0, \Sigma^{*}$ admits a Q-variety of moduli $\left(\mathscr{T}, \beta^{*}\right)$ defined over $k_{0}$ and $\mathscr{T}$ is a Q-manifold when the characteristic is 0 .

Proof. (a) and (b) follow from Propositions 14 and 15. We have settled (c) already.

By Proposition 17, there is a non-singular algebraic variety $A$ and a subvariety $Z$ of $A \times \boldsymbol{P}^{N}$, both defined over $k_{0}$, such that $u \times \boldsymbol{P}^{N}$ and $Z$ intersect properly on $A \times \boldsymbol{P}^{N}$ for every $u$ on $A$ and that the $Z(u)$ exhaust $\mathfrak{S}_{d}\left(N, n ; m_{1}, \ldots, m_{s}\right) . \quad \mathfrak{S}_{d}\left(N, n ; m_{1}, \ldots, m_{s}\right)$ satisfies the following conditions: (i) $l\left(C_{U}\right)=$ constant for all members $\boldsymbol{U}$; (ii) Every member $\boldsymbol{U}$ is not contained in a hyperplane; (iii) $C_{\boldsymbol{U}}^{(m)}$ is constant for all $\boldsymbol{U}$; (iv) The set 
of hyperplane sections of $\boldsymbol{U}$ is complete for all $\boldsymbol{U} ; \quad(\mathrm{v}) \mathfrak{B}\left(\boldsymbol{U} ; \mathbb{E}_{a}\left(C_{\boldsymbol{U}}\right)\right) \subset$ $\mathfrak{S}_{d}\left(N, n ; m_{1}, \ldots, m_{s}\right)$ for all $\boldsymbol{U}$. In fact, (i) follows from Proposition 13. (ii) is a part of our assumption. (iii) is obvious. (iv) follows easily from $h^{1}\left(\boldsymbol{U}, \mathfrak{V}_{\boldsymbol{U}}(m)\right)=0 . \quad$ (v) follows from (i) and (iv). Moreover, $\operatorname{dim} \mathfrak{p}\left(\boldsymbol{U}, \mathfrak{C}_{a}\left(C_{\boldsymbol{U}}\right)\right)$ $=\operatorname{dim} P G L(N)$ if $\rho \geqslant 0$. Assuming from now on that $\rho \geqslant 0$, Theorem $D$ shows that the equivalence relation on $\mathfrak{L}_{d}\left(N, n ; m_{1}, \ldots, m_{s}\right)$ defined in terms of isomorphisms of polarized varieties is strongly $k_{0}$-closed. Denote by $E_{0}$ the set of pairs of equivalent points on $A$ by the equivalence relation induced by that of $\mathfrak{h}_{d}\left(N, n ; m_{1}, \ldots, m_{s}\right)$. Since there is a canonical one-toone birational correspondence between $A$ and the Chow-variety of $\mathfrak{S}_{d}\left(N, n ; m_{1}, \ldots, m_{s}\right)$ defined over $k_{0}$ by Proposition $17, E_{0}$ is $k_{0}$-closed on $A \times A$ and the equivalence relation on $A$ is strongly $k_{0}$-closed. Hence $\left(A, E_{0}\right)$ defines a $Q$-variety $\mathscr{T}$, defined over $k_{0}$, consisting of regular points. Let $\beta$ be the canonical map of $A$ on $\mathscr{T} . \quad \beta$ is a morphism defined over $k_{0}$ and we extend $\beta$ to a map $\beta^{*}$ of $\Sigma^{*}$ onto $\mathscr{T}$ in the usual manner. Then it will be easy to verify that $\left(\mathscr{T}, \beta^{*}\right)$ is a $Q$-variety of moduli defined over $k_{0}$ if we show that $k_{0}(\beta(u))$ is the field of moduli of $Z(u)$ for all $u$ from $A$. The set $E_{0}\{u\}$ of points on $A$ which are equivalent to $u$ is the transform of $\mathfrak{p}\left(\boldsymbol{U}, \mathfrak{E}_{a}\left(C_{\boldsymbol{U}}\right)\right)$ by the canonical birational correspondence, if we set $Z(u)=\boldsymbol{U}$. $k_{0}(\beta(u))$ is by definition the smallest field of definition of the support of $E_{0}\{u\}$ over $k_{0}$. Hence it is the smallest field of definition of $\mathfrak{p}\left(\boldsymbol{U}, \mathfrak{S}_{a}\left(C_{\boldsymbol{U}}\right)\right)$ over k. $c_{0}$ is ample and $\mathscr{E}_{a}\left(C_{U}\right)^{+}$is a complete total family since it is the complete linear system determined by $C_{U}$. It follows that $k_{0}(\beta(u))$ is the field of moduli of $\boldsymbol{U}$ over $k_{0}$. (cf. Theorem 2). Our theorem is thereby proved.

\section{Appendix*)}

$\S 1$. Ruled varieties. A ruled variety $V$ is by definition an algebraic variety whose field of rational functions $K$ contains a subfield $L$ such that $K$ is a pure transcendental extension of positive dimension of $L$.

In this paragraph, we shall show that a non-singular variety in a projective space, which is a specialization of a ruled variety, is also a ruled variety. We shall begin with a rather obvious lemma, a proof of which will be left to the reader.

Lemma 1.1. Let $V$ be a non-singular ruled variety in a projective space. Then

*) Most of the results here are not new. They are collected here for the sake of convenience. 
$V \times V$ carries an irreducible algebraic family \&s of positive cycles $T$ with the following properties: (a) The Chow-variety of (s) is complete; (b) When $k$ is a common field of definition of $V$ and $\mathbb{S S}$ and when $T$ is a generic cycle of $\mathfrak{s}$ over $k, T$ is a birational correspondence between $V$ and $V ;$ (c) (5) contains a cycle $T^{\prime}$ such that every component of it is degenerate, i.e. no component $H$ of $T^{\prime}$ has the property $\left[H: V_{1}\right] \cdot\left[H: V_{2}\right] \neq 0$, where $V_{i}$ denotes the $i$-th factor of the product $V \times V$.

We shall state briefly an idea of a proof and leave the rest to the reader. There is a field $k$ of definition of $V$, a variety $W$ defined over $k$ and a rational map $f$ (not necessarily a morphism) of $V$ defined over $k$, generically surjective on $W$, such that a generic fibre $f^{-1}(w)$ over $k$ is birationally equivalent to the projective line over $k_{(}(w)$. One can find a family of positive chains satisfying (a), (b) and (c) on $f^{-1}(w) \times f^{-1}(w)$. From this family it is possible to construct a family of positive cycles on $V \times V$ we are looking for.

Theorem 1.1. Let $V$ be a non-singular ruled variety in a projective space, $V^{\prime}$ a variety in a projective space and $\mathfrak{D}$ a discrete valuation-ring such that $V^{\prime}$ is a specialization of $V$ over 5 . Then $V^{\prime}$ is also a ruled variety.

Proof. By our lemma, $V \times V$ carries an irreducible algebraic family \&s of positive cycles satisfying (a), (b), (c). Let $G$ be the support of the Chowvariety of (S) and $(V, G) \rightarrow\left(V^{\prime}, G^{\prime}\right)$ ref. 5 . Since $G$ is connected, $G^{\prime}$ is connected by Zariski's connectedness theorem, extended by Chow and Grothendieck (cf. [1], [3]).

Assume that $V^{\prime}$ is not ruled. Let $v^{\prime}$ be a point of $G^{\prime}$. There is a point $v$ of $G$ such that $(V, G, v) \rightarrow\left(V^{\prime}, G^{\prime}, v^{\prime}\right)$ ref. $D$ and we may take $v$ to be a generic point of $G$ over a common field of definition of $V$ and $G$. $v$ is then the Chow-point of a birational correspondence between $V$ and $V$. When that is so, $v^{\prime}$ is the Chow-point of a positive chain on $V^{\prime} \times V^{\prime}$, which contains a component such that it is a birational correspondence between $V^{\prime}$ and $V^{\prime}$ (cf. [16]). Since \&s contains a degenerate member by (c), it follows that $G^{\prime}$ contains the Chow-point of a degenerate chain on $V^{\prime} \times V^{\prime}$. This is against to what we have observed and $V^{\prime}$ has to be a ruled variety.

\section{§ 2. Upper semi-continuity.}

Proposition 2.1. Let $V, W$ be non-singular algebraic varieties in projective 
spaces, $X$ a V-divisor, $Y$ a W-divisor and $\subseteq$ a discrete valuation-ring such that $(V, X) \rightarrow\left(W^{\prime}, Y\right)$ ref. 5 . Then

$$
h^{i}(V, X) \leqslant h^{i}(W, Y)
$$

Proof. This is well-known and is a special case of a more general upper semi-continuity (cf. [3]). But if a reader is interested in proving this case as a simple exercise, he can proceed as follows. First observe that the case of dimension 1 is an easy consequence of the Riemann-Roch theorem, the formula $l(X) \leqslant l(Y)$ and the invariance of canonical divisors by specializations. In general, denote by $C_{m}$ a hypersurface section of $V$ by a hypersurface of degree $m$ so that $C_{m}$ is a normal variety (cf. [18]). Then $h^{i+1}(V, X)=$ $h^{i}\left(C_{m}, C_{m} \cdot\left(C_{m}^{\prime}+X\right)\right)$ for $i>0$ if $m$ is large (cf. [26]). Then the general case can be proved by induction, using the invariance of the Euler-Poincaré characteristic (cf. Theorem $A$ ), the duality theorem and $l(X) \leqslant l(Y)$.

Proposition 2.2. Let $V, W$ be non-singular subvarieties of projective spaces, $X$ a V-divisor and $Y$ a W-divisor. Let $\$$ be a discrete valuation-ring and assume that $(W, Y)$ is a specialization of $(V, X)$ over 5 and $l(X)=l(Y)$. Then, when $Y$ is ample, $X$ is also ample.

Proof. Let $G(X)$ (resp. $G(Y)$ ) be the support of the Chow-variety of $\Lambda(X)$ (resp. $\Lambda(Y)$ ). Since linear equivalence is preserved by specializations, it follows that $(V, X, G(X)) \rightarrow(W, Y, G(Y))$ ref. $\mathfrak{D}$. Therefore, when $\left(v_{1}, \ldots, v_{m}\right)$ is a set of points of $G(Y)$, there is a set $\left(u_{1}, \ldots, u_{m}\right)$ of points in $G(X)$ such

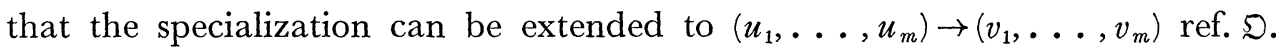

Since $Y$ is ample, $\Lambda(Y)$ has the property that (i) it separates points of $W$ and that (ii) for any given point $Q$ of $W$ there is a set $Y_{1}, \ldots, Y_{n}, n=$ $\operatorname{dim} W$, of members of $\Lambda(Y)$ such that $Q$ is a proper component of multiplicity 1 of $\cap_{i} Y_{i}$ on $W$. Conversely, since (ii) implies that $Q$ is simple on the $Y_{i}$ and that the tangent linear spaces to the $Y_{i}$ at $Q$ form a set of $n$ independent hyperplanes on the tangent linear space to $W$ at $Q$, (i) and (ii) imply the ampleness of $Y$. Using this remark and also the above remark for specializations, we can verify easily that $X$ satisfies (i) and (ii) and hence that $X$ is ample on $V$. In order to do this, what we need is the compatibility of specializations with intersection-product and a few fundamental facts on specializations of cycles (cf. [21]). Details will be left to the reader.

Proposition 2.3. Using the same notations and assumptions as in Proposition 
2.2, assume further that $Y$ is sufficiently ample. Then $X$ is sufficiently ample.

Proof. This is an immediate consequence of Propositions 2.1 and 2.2 since algebraic equivalence is preserved by specializations.

§ 3. Some cohomological questions.

Proposition 3.1. Let $V^{n}$ be a non-singular subvariety of a projective space and $X, Z$ divisors on $V$. Then $\chi(V, X+m Z)$ is a polynomial in $m$.

This is a special case of a theorem of Snapper (cf. [22']) and a little more general result is obtained in $\left[8^{\prime}\right]$. But it is also an easy exercise to the readers. Note that $\chi(V, D)=(-1)^{n}\left(p_{a}(V)+p_{a}(-D)\right)$ (cf. [26]), that $V$ carries an ample divisor and then make use of the formula for $p_{a}$ which is similar to [46], $\S 12$ in [27]. As a corollary of this, Theorem $C$ and Theorem $A$, we get the following result.

Proposition 3.2. Let $V$ be a non-singular subvariety of a projective space and $X, Y$ two divisors on $V$ which are numerically equivalent on $V$. Then $\chi(V, m X)$ $=\chi(V, m Y)$ for all integers $m$.

Proposition 3.3. Let $V^{n}$ be a non-singular subvariety of a projective space, $\Lambda^{*}$ an ample linear system on $V$ and $X$ a divisor on $V$ such that $\Lambda(X)$ is not empty. Let $C^{(i)}$ be a non-singular subvariety of $V$ of codimension $i$, which is the intersection-product of $i$ properly intersecting members of $\Lambda^{*}$, such that $X$ and the $C^{(i)}$ intersect properly on $V$. Assume that the following conditions are satisfied: (a) $\Lambda(X)$ induces on $C^{(i)}$ the complete linear system $\Lambda_{i}=\Lambda\left(X \cdot C^{(i)}\right)$ for $i>0$; (b) $\Lambda^{\prime}=\Lambda(X)-\Lambda^{*}$ exists and induces on $C^{(n-1)}$ a complete non-special linear system. Then the minimum sum of $\Lambda^{*}$ and $\Lambda(X)$ is complete and $\Lambda(X+C)$ induces a complete linear system on $C$.

Proof. We proceed by induction on $n$. A well-known lemma of Castelnuovo states that when $A^{*}$ is a linear system without base points on a complete non-singular curve and when a complete non-special linear system $\Lambda$ is such that $\Lambda-\Lambda^{*}$ exists and non-special, then the minimum sum of $\Lambda^{*}$ and $\Lambda$ is complete. When $n=1$, our assumptions imply that these conditions are satisfied. Hence the minimum sum of $\Lambda^{*}$ and $\Lambda(X)$ is complete.

Assume now that our proposition is true up to dimensions $n-1$. Set $C^{(1)}=C . \Lambda(X)$ induces $\Lambda(X \cdot C)$ on $C$ and $A^{*}$ induces on $C$ an ample linear system $\Lambda_{1}^{*}$. Moreover, $\Lambda(X \cdot C)-\Lambda_{1}^{*}$ exists and this induces $\operatorname{Tr}_{C}^{(n-1)}\left(\Lambda(X)-\Lambda^{*}\right)$. 
Therefore, the minimum sum of $\Lambda(X \cdot C)$ and $\Lambda_{1}^{*}$ is complete by our induction assumption. Let $C^{\prime}$ be a member of $\Lambda_{1}^{*}$ such that $C$ and $C^{\prime}$ intersect properly on $V$. Let $M$ be the defining module of functions $h$ of $A^{*}$ on $V$ such that $\operatorname{div}(h)+C^{\prime}>0$. When the $f$ are taken from $L(X)$ and the $h$ taken from $M$, the functions $f \cdot h$ generate the module $M^{\prime}$ of functions which defines the minimum sum $\Lambda^{* \prime}$ of $\Lambda(X)$ and $\Lambda^{*} . \operatorname{Tr}_{C} \Lambda^{* \prime}$ contains clearly the minimum sum of $T r_{C} \Lambda(X)$ and $T r_{C} \Lambda^{*}$. Hence $T r_{C} \Lambda^{* \prime}=\Lambda\left(C \cdot\left(X+C^{\prime}\right)\right)$. It follows that $M^{\prime}$ induces the module $L\left(C \cdot\left(X+C^{\prime}\right)\right)$ on $C . \quad M^{\prime}$ is a submodule of $L\left(X+C^{\prime}\right)$. Hence we can complete a basis of $M^{\prime}$ to a basis of $L\left(X+C^{\prime}\right)$ by adding those functions $g$ on $V$ which induce 0 on $C$. Since $\operatorname{div}(g)=Y-X-C^{\prime}, Y>0$, and since $C$ is not a component of $X$ and $C^{\prime}$, it follows that $C$ is a component of $Y$. Set $Y=Y^{\prime}+C$. $Y^{\prime}$ is a positive divisor. Let $h$ be a function in $M$ such that $\operatorname{div}(h)=C-C^{\prime}$. Then $g / h$ is in $L(X)$ and hence $g$ is in $M^{\prime}$. Our proposition is thereby proved.

Proposition. 3.4. Let $V^{n}$ be a non-singular subvariety of a projective space, $X$ a divisor on $V$ and $Y$ an ample divisor on $V$. Let $Y^{(\alpha)}$ be a non-singular subvariety of $V$ of codimension $\alpha$, which is the intersection-product of $\alpha$ properly intersecting members of $\Lambda(Y)$ such that $X$ and the $Y^{(\alpha)}$ intersect properly on $V$. When $h^{i}(V, X)$ $=0, h^{i}\left(Y^{(\alpha)}, X \cdot Y^{(\alpha)}\right)=0$ for $i>0$ and $1 \leqslant \alpha \leqslant n-1$, then $h^{2}(V, X+m Y)=0$ for $i>0, m>0$.

Proof. When $n=1$, our result follows from the theorem of RiemannRoch. Therefore, we proceed by induction on $n$. Let $Y^{\prime}$ be a member of $\Lambda(Y)$ such that $Y^{\prime}$ and the $Y^{(\alpha)}$ intersect properly on $V$. Setting $Y^{(1)}=Y$, our induction assumption and the cohomology exact sequence from $0 \rightarrow \mathfrak{R}\left(V, X+(m-1) Y^{\prime}\right) \rightarrow \mathfrak{L}\left(V, X+m Y^{\prime}\right) \rightarrow \mathfrak{R}\left(Y, Y \cdot\left(X+m Y^{\prime}\right)\right) \rightarrow 0$ imply $h^{i}\left(V, X+(m-1) Y^{\prime}\right) \geqslant h^{i}\left(V, X+m Y^{\prime}\right)$ for $i>0, m>0$. Since $h^{i}(V, X)=0$ for $i>0$ by our assumption, we get our lemma from this.

Proposition 3.5. Let $V^{n}$ be a non-singular subvariety of a projective space, $\Lambda$ an ample linear system on $V$ and $X$ a divisor on $V$. Let $C$ be a member of $\Lambda$ and set $\chi(V, m C)=g(m), \chi(V, X+m C)=g_{X}(m)$. Then there is a constant $t_{0}$, depending only on $g(x), g_{X}(x)$ and the intersection numbers $c_{j}=I\left(X^{(j)} \cdot C^{(n-j)}\right)$, such that $h^{i}(V, X+t C)=0$ for $i>0$ when $t>t_{0}$.

Proof. Let $C^{(\alpha)}$ be a non-singular subvariety of $V$ of codimension $\alpha$, 
which is the intersection-product of $\alpha$ properly intersecting members of $\Lambda$, such that $\left(X, C^{(\alpha)}\right),\left(C^{(\alpha)}, C^{(\beta)}\right)$ are pairs of properly intersecting cycles on $V$ and that $C^{(\alpha)} \cdot C^{(\beta)}=C^{(\alpha+\beta)}$ provided $\alpha+\beta \leqslant n$. Set $C^{(1)}=C$. $\chi\left(C^{(\alpha)}\right.$, $\left.X \cdot C^{(\alpha)}+(m-1) C \cdot C^{(\alpha)}\right)+\chi\left(C^{(\alpha+1)}, X \cdot C^{(\alpha+1)}+m C \cdot C^{(\alpha+1)}\right)=\chi\left(C^{(\alpha)}, X \cdot C^{(\alpha)}+m C \cdot C^{(\alpha)}\right)$ implies that the polynomial $g_{X} \cdot c^{(\alpha)}(m)=\chi\left(C^{(\alpha)}, X \cdot C^{(\alpha)}+m C \cdot C^{(\alpha)}\right)$ can be deduced from $g_{X}(m)$ for $1 \leqslant \alpha \leqslant n-1$. In the same way, the polynomial $g_{\alpha}(m)=\chi\left(C^{(\alpha)}, m C \cdot C^{(\alpha)}\right)$ can be deduced from $g(m)$ for $1 \leqslant \alpha \leqslant n-1$.

When $n=1$, our result follows at once from the theorem of Riemann-Roch. Therefore, assume that there is a constant $t^{\prime}$, depending only on $g(x), g_{X}(x)$ and the $c_{j}$, such that $h^{i}\left(C^{(\alpha)}, C^{(\alpha)} \cdot(X+t C)\right)=0$ for $i>0$ and $1 \leqslant \alpha \leqslant n-1$ when $t \geqslant t^{\prime}$. The exact cohomology sequence of $0 \rightarrow \mathfrak{Q}\left(V, X+(t-1) C^{\prime}\right) \rightarrow \mathfrak{L}\left(V, X+t C^{\prime}\right) \rightarrow \mathfrak{L}\left(C, C \cdot\left(X+t C^{\prime}\right)\right) \rightarrow 0$ and the induction assumption imply $h^{1}(V, X+(t-1) C) \geqslant h^{1}(V, X+t C)$ and $h^{i}(V, X+(t-1) C)=$ $h^{i}(V, X+t C)$ for $t \geqslant t^{\prime}, i>1 \quad\left(C^{\prime}\right.$ is a member of $\Lambda$ such that $C$ and $C^{\prime}$ intersect properly on $V)$. Since $h^{i}(V, X+t C)=0$ for $i>0$ and for large $t$, we see first of all that $h^{i}(V, X+t C)=0$ for $i>1$ and $t \geqslant t^{\prime}$. Suppose, for some $t \geqslant t^{\prime}$, that $h^{1}(V, X+(t-1) C)=h^{1}(V, X+t C)=h^{1}(V, X+(t+1) C)$. Then the exact cohomology sequence we quoted above implies that $\Lambda(X+t C)$ and $\Lambda(X+(t+1) C)$ induce on $C$ complete linear systems. When that is so, these two complete linear systems on $V$ induce complete linear systems on $C^{(\alpha)}$ by similar reasons as above and by our induction assumption. Therefore $\Lambda$ and $\Lambda(X+(t+1) C)$ satisfy the requirements of Proposition 3.3. It follows that the minimum sum of these linear systems is $\Lambda(X+(t+2) C)$. The same is true of the induced linear systems of $A$ and $A(X+(t+1) C)$ on $C$, and consequently the minimum sum of these two on $C$ is also complete. It follows that $\Lambda(X+(t+2) C)$ induces on $C$ a complete linear system. When we replace $t$ by $t+2$ in the above exact sequence of sheaves and apply the induction assumption and this result to the exact cohomology sequence derived from it, we see that $h^{1}(V, X+(t+1) C)=h^{1}(V, X+(t+2) C)$. Repeating this process, we get $h^{1}(V, X+(t-1) C)=h^{1}(V, X+(t+l) C)$ for all nonnegative $l$. This is impossible unless $h^{1}(V, X+(t-1+l) C)=0$ for all non-negative $l$.

On the other hand, from what we have proved and from the definition, we see that $h^{0}\left(V, X+t^{\prime} C\right)=g_{X}\left(t^{\prime}\right)+h^{1}\left(V, X+t^{\prime} C\right)$. Moreover, we have $h^{0}\left(V, X+t^{\prime} C\right) \leqslant\left(X+t^{\prime} C\right)^{(n)}+n$ (cf. [16]). From this we get $h^{1}\left(V, X+t^{\prime} C\right) \leqslant$ $\left(X+t^{\prime} C\right)^{(n)}+n-g_{X}\left(t^{\prime}\right)=s$. Then it is now an easy exercise to show that 
$h^{1}(V, X+t C)=0$ whenever $t>t^{\prime}+2 s+1$. Our proposition is thereby proved.

\section{§ 4. On algebro-geometric conditions.}

Proposition 4.1. Let $V$ be a closed subset of an algebraic variety $A$ and (P) a set of algebro-geometric conditions. Assume that the following properties are satisfied by $V$ and $(P)$. (a) When $W$ is a subvariety of $A$, contained in $V$ and defined over a field $k$ of definition of $A$ over which $V$ is closed, and when there is a generic point of $W$ over $k$ which satisfies $(P)$, there is an open subset $D$ of $W$, containing this point, such that every point of $D$ satisfies $(P) . \quad(\mathrm{b})$ There is a field $K$ of definition of $A$ over which $V$ is closed with the property that, when $x, x^{\prime}$ are points of $V, x \rightarrow x^{\prime}$ ref. $K$ and when $x^{\prime}$ satisfies $(P)$, then $x$ satisfies $(P)$. (c) A generic point of each component of $V$ over $\bar{K}$ satisfies $(P)$. Then the set of points $U$ of $V$ which satisfies $(P)$ is a $K$-open subset of $V$.

Proof. It is easy to see that our proposition can be deduced from the case when $V$ is a subvariety of $A$ defined over $\bar{K}$. Therefore, we shall assume that $V$ is a subvariety of $A$ defined over $K$.

Assume now that $x$ satisfies $(P)$ and let $D$ be an open subset of $V$, containing $x$, such that every point of it is a point of $U$. $D$ exists by (a). Let the $W_{\alpha}$ be the components of $V-D$ containing some points of $U$ and the $W_{\beta}$ the components of $V-D$ which do not contain any point of $U$. Set $V-U_{\beta} W_{\beta}=V^{\prime}$ and let $D^{\prime}, U^{\prime}$ be the restrictions of $D, U$ on $V^{\prime} . V^{\prime}$ is open on $V$ and $D=D^{\prime}, U=U^{\prime}$. Therefore, it is enough to prove that $U$ is open on $V^{\prime}$. When that is so, we may assume without loss of generality that $V=V^{\prime}$. By (a) and (b), there is a non-empty open subset $D_{\alpha}$ of $W_{\alpha}$ such that every point of it is contained in $U$. Let the $W_{\alpha, \gamma}$ be the components of $W_{\alpha}-D_{\alpha}$ and $D^{(1)}$ the open subset $V-\cup_{\alpha, \gamma} W_{\alpha, \gamma} . \quad D$ is contained strictly in this open subset and every point of $D^{(1)}$ is contained in $U$. When we continue this process, we get an increasing sequence of open subsets of $V$ which are contained in $U$. Moreover, this does not terminate as long as points of $U$ are not exhausted. Therefore, $U$ is an open subset of $V$.

When that is so, $U$ is a $K$-open subset of $V$ by the definition of $U$ and by our assumption (b). Our proposition is thereby proved.

In order to discuss the following lemmas and a proposition, we shall 
fix some notations and conventions. Let $\mathfrak{h}$ be an irreducible algebraic family of non-singular varieties in a projective space, $k$ a field of definition of $\mathfrak{S}$ and $H$ the Chow-variety of $\mathfrak{S}$. Let $s$ be a positive integer, which is prime to the characteristic, such that $s$ and $\mathrm{t}\left(V^{\prime}\right)$ are relatively prime for all $V^{\prime}$ in $\mathfrak{5}$. We set $r=s$ or $1 / s$. We denote by $r \widetilde{C}_{a}\left(C_{V^{\prime}}\right)$ the class $\mathfrak{C}_{a}\left(s C_{V^{\prime}}\right)$ if $r=s$ and the class $\mathfrak{E}_{a}\left(X^{\prime}\right)$ where $X^{\prime}$ is a $V^{\prime}$-divisor such that $s X^{\prime} \equiv C_{V^{\prime}} \bmod \mathbb{S}_{a}$ if $r=1 / s$. In Lemmas $4.1,4.2$ we shall assume that a sufficiently ample divisor is contained in $r \mathfrak{S}_{a}\left(C_{V^{\prime}}\right)$ for every $V^{\prime} \in \mathfrak{S}$. When that is so, every divisor in $r \widetilde{\varepsilon}_{a}\left(C_{V^{\prime}}\right)$ is sufficiently ample and $r \widetilde{\varepsilon}_{a}\left(C_{V^{\prime}}\right)^{+}$is a complete total family defined over $k\left(v^{\prime}\right)$ if $v^{\prime}=\mathfrak{c}\left(V^{\prime}\right)$ (cf. Proposition 2 and Theorems $E$ and $G$ ).

Let $v$ be a generic point of $H$ over $k$, representing $V \in \mathfrak{K}, X$ a generic divisor of $r \widetilde{S}_{a}\left(C_{V}\right)^{+}$over $k(v)$ and $K$ a field of rationality of $X$ over $k(v)$. Let $\boldsymbol{f}_{X}$ be a non-degenerate projective embedding of $V$ determined by $X$, rational over $K$, and $\tau$ a generic projective transformation over $K$ of the ambient space of $\boldsymbol{f}_{X}(V) . \quad$ Set $\quad \Gamma=\Gamma_{\tau} \circ \boldsymbol{f}_{X}, \quad u=\mathfrak{c}\left(\tau \circ \boldsymbol{f}_{X}(V)\right), \quad t=\mathfrak{c}(\Gamma), \bar{G}=$ $\operatorname{loc}_{\bar{k}}(u), \bar{J}=\operatorname{loc}_{\bar{k}}(t), \bar{T}=\operatorname{loc}_{\bar{k}}(v, t, t)$.

Lemma 4.1. (a) $\bar{G}, \bar{J}, \bar{T}$ are defined over to. (b) The set of points $G$ in $\bar{G}$ which represent non-singular varieties, not contained in any hyperplane, is k-open on $\vec{G}$. (c) The set of points $J$ in $\vec{J}$ which represent irreducible cycles in the multiple projective space is k-open on $\vec{J}$. (d) The restriction $T$ of $\bar{T}$ on $H \times G \times J$ is k-open on $\bar{T}$.

Proof. (a) follows easily from the fact that $K$ can be taken as a regular extension of $k(v)$. (b) and (c) follows from the fact that those properties can be described in terms of non-vanishing of finite sets of homogeneous polynomials over $k$ in the Chow-coordinates. (d) follows at once from (b) and (c).

Lemma 4.2. A point $\left(v^{\prime}, u^{\prime}, t^{\prime}\right)$ is in $T$ if and only if $v^{\prime}$ represents a member $V^{\prime}$ of $\mathfrak{S}, t^{\prime}$ represents a non-degenerate projective embedding $\boldsymbol{f}_{X^{\prime}}$ of $V^{\prime}$ with $X^{\prime} \in r \varsigma_{a}\left(C_{V^{\prime}}\right)^{+}$and $u^{\prime}$ represents $\boldsymbol{f}_{X^{\prime}}\left(V^{\prime}\right)$.

Proof. Let $\left(v^{\prime}, u^{\prime}, t^{\prime}\right)$ be a point of $T$. It is a specialization of $(v, u, t)$ over $k$ and, when that is so, $v^{\prime}, u^{\prime}, t^{\prime}$ are as described in our lemma by Propositions 2, 4, Theorem $D^{\prime}$ and by the compatibilities of specializations with the operations of intersection-product and algebraic projection. Assume now that $V^{\prime}$ is in $\mathfrak{S}, u^{\prime}$ represents $\boldsymbol{f}_{X}\left(V^{\prime}\right)$ and $t^{\prime}$ represents the graph of $\boldsymbol{f}_{X^{\prime}}$ with $X^{\prime} \in r \mathfrak{c}_{a}\left(C_{V^{\prime}}\right)^{+} . \quad V^{\prime}$ is a specialization of $V$ over $k$ and there is a 
divisor $\tilde{X}$ in $r \widetilde{E}_{a}\left(C_{V}\right)^{+}$such that $(V, \tilde{X}) \rightarrow\left(V^{\prime}, X^{\prime}\right)$ ref. $k$ by Propositions 2 and 4. Then $(V, X) \rightarrow\left(V^{\prime}, X^{\prime}\right)$ ref. $k$ by our choice of $X$. By Propositions 3 and 6 , there is the graph $\Gamma^{\#}$ of a non-degenerate projective embedding of $V$, determined by $X$, such that $\left(V, X, \Gamma^{\sharp}\right) \rightarrow\left(V^{\prime}, X^{\prime}, \Gamma_{f_{X^{\prime}}}\right)$ ref. $k$. From the definition of $\Gamma$, it follows easily that $\left(V, X, \Gamma^{\sharp}\right)$ is a specialization of $(V, X, \Gamma)$ over $k(v)$. Hence $\left(v^{\prime}, u^{\prime}, t^{\prime}\right)$ is a point of $T$ and our lemma is thereby proved.

Proposition 4.2. Let $\mathfrak{h}$ be an irreducible algebraic family of non-singular varieties in a projective space $\boldsymbol{P}$. Let $s$ be a positive integer which is prime to the characteristic and to the order $\mathfrak{t}(V)$ of a member $V$ of $\mathfrak{S}$. Setting $r=s$ or $r=1 / s$, assume that the following conditions are satisfied: (a) For any member $V$ of $\mathfrak{S}$, the set of hyperplane sections of $V$ forms a complete linear system; (b) For such $V, \mathbb{S}_{a}\left(C_{V}\right)$ and $r \mathfrak{S}_{a}\left(C_{V}\right)$ contain sufficiently ample divisors on $V ;$ (c) $\mathfrak{S}$ contains $\mathfrak{P}\left(V, \mathfrak{C}_{a}\left(C_{V}\right)\right)$ for all $V$ in $\mathfrak{S}$. Then, when we set $\mathfrak{S}_{r}=\cup V \in \mathfrak{g} \mathfrak{H}\left(V, r \mathfrak{C}_{a}\left(C_{V}\right)\right), \mathfrak{S}_{r}$ is an irreducible algebraic family. When $\mathfrak{S}$ is defined over a field $k$, so is $\mathfrak{H}_{r}$.

Proof. By Theorem $F, s$ is prime to $t(V)$ for every $V$ in $\mathfrak{H}$. Let $H$ be the Chow-variety of $\mathfrak{S}$ and let us use the same notations and conventions of Lemmas 4.1 and 4.2. By Lemma 4.2, when we denote by $H_{r}$ the settheoretic projection of $T$ on $G, H_{r}$ is the set of points representing members of $\mathfrak{K}_{r}$. Therefore, we must show that $H_{r}$ is a $k$-open subset of $G$. In order to do this, we shall show that the conditions (a), (b), (c) of Proposition 4.1 are satisfied.

(c) is trivially satisfied in our case. Let $G^{\prime}$ be a subvariety of $G$ such that $G^{\prime} \cap H_{r}$ contains a generic point of $G^{\prime}$ over a field $L$ of definition of $G^{\prime}$ containing $k$. Then by Lemma 4.2, $H \times G^{\prime} \times J \cap T$ contains a component $T^{\prime}$ which has the projection $G^{\prime}$ on $G$. Hence $G^{\prime} \cap H_{r}$ contains an $L$-open subset of $G^{\prime}$, containing the generic point of $G^{\prime}$ over L. (cf. [24]). Thus we have verified the condition (a).

Next we shall verify the condition (b) with respect to the field $k$. Let $u^{*}, u^{\prime}$ be points of $G$, corresponding to non-singular varieties $U^{*}, U^{\prime}$ such that $u^{*} \rightarrow u^{\prime}$ ref. $k$. Assume that $U^{\prime}$ is a member of $\mathfrak{S}_{r}$. There is a member $V^{\prime}$ of $\mathfrak{Y}$ and a non-degenerate projective embedding $\boldsymbol{f}^{\prime}$ of $V^{\prime}$ such that $\boldsymbol{f}^{\prime-1}\left(C_{U^{\prime}}\right) \in r \mathbb{E}_{a}\left(C_{V^{\prime}}\right)$. Set $\tau \circ \boldsymbol{f}_{X}(V)=U \quad$ and $\tau \circ \boldsymbol{f}_{X}\left(C_{V}\right)=Y$. Let $\langle U, Y) \rightarrow\left(U^{*}, Y^{*}\right)$ ref. $k$ and $\left(U^{*}, Y^{*}\right) \rightarrow\left(U^{\prime}, Y^{\prime}\right)$ ref. $k$. Since $C_{U} \in r \widetilde{\varepsilon}_{a}(Y)$ and since algebraic equivalence is preserved by specializations, it follows that 
$C_{U^{*}} \in r \aleph_{a}\left(Y^{*}\right)$ and $C_{U^{\prime}} \in r \aleph_{a}\left(Y^{\prime}\right)$. When that is so, $r \Im_{a}\left(\boldsymbol{f}^{\prime-1}\left(Y^{\prime}\right)\right)=r \aleph_{a}\left(C_{V^{\prime}}\right)$ and consequently $\boldsymbol{f}^{\prime-1}\left(Y^{\prime}\right) \equiv C_{V^{\prime}} \bmod \mathscr{S}_{a}$ by our choice of $r$. Hence $Y^{\prime}$ is sufficiently ample and $Y^{*}$ is also sufficiently ample by Proposition 2.3. Moreover, $l(Y)=l\left(Y^{*}\right)=l\left(Y^{\prime}\right)$ by Proposition 3. Set $\boldsymbol{f}_{Y^{\prime}}\left(U^{\prime}\right)=\tilde{V}^{\prime}$. By the definition, we have $\boldsymbol{f}_{Y \prime}\left(Y^{\prime}\right) \equiv C_{\tilde{V}^{\prime}} \bmod \mathscr{S}_{a}$. Hence $\boldsymbol{g}^{\prime}=\boldsymbol{f}_{Y^{\prime}} \circ \boldsymbol{f}^{\prime}$ is a nondegenerate projective embedding of $V^{\prime}$, determined by a divisor in $\mathfrak{S}_{a}\left(C_{V^{\prime}}\right)$, i.e. $\tilde{V}^{\prime} \in \mathfrak{P}\left(V^{\prime}, \mathfrak{C}_{a}\left(C_{V^{\prime}}\right)\right)$. It follows that $\tilde{V}^{\prime} \in \mathfrak{S}$ by our assumption. Applying Proposition 6 to $\boldsymbol{f}_{Y^{\prime}}$ and to $\left(U^{*}, Y^{*}\right) \rightarrow\left(U^{\prime}, Y^{\prime}\right)$ ref. $k$, we see the existence of a non-degenerate projective embedding $\boldsymbol{f}^{*}$ of $U^{*}$, determined by $Y^{*}$, such that $\left(U^{*}, Y^{*}, \Gamma_{\boldsymbol{f}^{*}}\right) \rightarrow\left(U^{\prime}, Y^{\prime}, \Gamma_{\boldsymbol{f}^{\prime}}\right)$ ref. $k$. Doing the same to $\Gamma_{\boldsymbol{f}^{*}}$ and to $(U, Y)$ $\rightarrow\left(U^{*}, Y^{*}\right)$ ref. $k$, we see the existence of the graph $\tilde{\Gamma}$ of a non-degenerate projective embedding of $U$, determined by $Y$, such that $(U, Y, \tilde{\Gamma}) \rightarrow$ $\left(U^{*}, Y^{*}, \Gamma_{\boldsymbol{f}^{*}}\right)$ ref. $k . \quad\left(\tau \circ \boldsymbol{f}_{X}\right)^{-1}$ is a non-degenerate projective embedding of $U$, determined by $Y$. Therefore, when we put $\tilde{V}=\tilde{\Gamma}(U), V$ and $\tilde{V}$ differ by a projective transformation and $\tilde{V}$ is a member of $\mathfrak{P}\left(V, \mathfrak{c}_{a}\left(C_{V}\right)\right)$. When we set $\boldsymbol{f}^{*}\left(U^{*}\right)=V^{*}$, we have $\tilde{V} \rightarrow V^{*}$ ref. $k$ and $V^{*} \rightarrow \tilde{V}^{\prime}$ ref. $k$. Since $\tilde{V}, \tilde{V}^{\prime} \in \mathfrak{H}$ as we have seen and since $\mathfrak{H}$ is an algebraic family defined over $k$, it follows that $V^{*} \in \mathfrak{h}$. Since $\tilde{\Gamma}^{-1}$ is determined by a divisor in $r \widetilde{C}_{a}\left(C_{\tilde{V}}\right)$, it follows that $\Gamma_{\boldsymbol{f}^{*}}{ }^{-1}$ is defined by a divisor in $r \mathbb{S}_{a}\left(C_{V^{*}}\right)$ by the compatibility of specializations with intersection-product and algebraic projection. This shows, by Lemma 4.2 , that $U^{*}$ is a member of $\mathfrak{S}_{r}$. The condition (b) is thus verified and our proposition is thereby proved.

COROLlaRy. Let $\mathfrak{S}$ be an algebraic family of non-singular varieties in a projective space. Let $r$ be as in our proposition. Assume that $\mathfrak{H}$ satisfies (a) and (b) of our proposition and also the following condition: ( $\left.\mathbf{c}^{\prime}\right)$ When $V$ is contained in a component $\mathfrak{H}_{\alpha}$ of $\mathfrak{S}, \mathfrak{S}_{\alpha}$ contains $\mathfrak{B}\left(V, \mathfrak{S}_{a}\left(C_{V}\right)\right)$. Then, when we define $\mathfrak{S}_{r}$ as in our proposition, it is an algebraic family. Moreover, when $\mathfrak{S}$ is normally algebraic over a field $k$, so is $\mathfrak{\mathfrak { L }}_{r}$.

Proof. Let the $\mathfrak{S}_{\alpha}$ be the component families of $\mathfrak{S}$. Then $\mathfrak{S}_{r}$ is the union of the $\left(\mathfrak{S}_{\alpha}\right)_{r}$, which is an algebraic family defined over $\bar{k}$ by our proposition. Let the $\left(H_{\alpha}\right)_{r}$ be the Chow-variety of $\left(\mathfrak{S}_{\alpha}\right)_{r}$ and $S_{\alpha}$ the complement of $\left(H_{\alpha}\right)_{r}$ in its closure. Let $\widetilde{S}_{\alpha}$ be the algebraic family of positive cycles in the projective space defined by $S_{\alpha}$. Our corollary will follow easily if we show that $\mathfrak{S}_{\alpha} \cap\left(\mathfrak{S}_{\beta}\right)_{r}=\phi$ when $\alpha \neq \beta$.

Assume that $\mathfrak{S}_{\alpha} \cap\left(\mathfrak{S}_{\beta}\right)_{r}$ contains $U^{\prime}$. Let $U^{*}$ be a generic member of 
a component family of $\mathfrak{S}_{\alpha}$ over $\bar{k}$ which contains $U^{\prime}$. Let $U$ be a generic member of $\left(\mathfrak{S}_{\alpha}\right)_{r}$ over $\vec{k}$. There is a generic member $V$ of $\mathfrak{S}_{\alpha}$ over $\vec{k}$ and an isomorphism $\boldsymbol{f}$ between $V$ and $U$ such that $\boldsymbol{f}^{-1}\left(C_{U}\right) \in r \mathbb{\varepsilon}_{a}\left(C_{V}\right)$. Set $\boldsymbol{f}\left(C_{V}\right)=Y$. Let $(U, Y) \rightarrow\left(U^{*}, Y^{*}\right)$ ref. $\bar{k}$ and $\left(U^{*}, Y^{*}\right) \rightarrow\left(U^{\prime}, Y^{\prime}\right)$ ref. $\bar{k}$. Since algebraic equivalence is preserved by specializations, it follows that $C_{U} \in r \Im_{a}(Y), C_{U^{*}} \in r \mathfrak{\Im}_{a}\left(Y^{*}\right)$ and $C_{U^{\prime}} \in r \mathfrak{\Im}_{a}\left(Y^{\prime}\right)$. Since $U^{\prime}$ is a member of $\left(\mathfrak{S}_{\beta}\right)_{r}$, there is a member $V^{\prime}$ of $\mathfrak{S}_{\beta}$ and an isomorphism $\boldsymbol{f}^{\prime}$ between $V^{\prime}$ and $U^{\prime}$ such that $\boldsymbol{f}^{\prime-1}\left(C_{U^{\prime}}\right) \in r \widetilde{C}_{a}\left(C_{V^{\prime}}\right)$. When that is so, $\boldsymbol{f}^{\prime-1}\left(Y^{\prime}\right) \equiv C_{V^{\prime}} \bmod \mathscr{S}_{a}$ by the definition of $r$ and $Y^{\prime}$ is sufficiently ample. Therefore, $Y^{*}$ is also sufficiently ample by Proposition 2.3. Then, as exactly the same way as in the last part of the proof of our proposition, we can find the graphs $\tilde{\Gamma}, \Gamma^{*}, \Gamma^{\prime}$ of non-degenerate projective embeddings of $U, U^{*}, U^{\prime}$, determined respectively by $Y, Y^{*}, Y^{\prime}$ with the following properties: (i) $(U, Y, \tilde{\Gamma}) \rightarrow$ $\left(U^{*}, Y^{*}, \Gamma^{*}\right)$ ref. $\bar{k}$ and $\left(U^{*}, Y^{*}, \Gamma^{*}\right) \rightarrow\left(U^{\prime}, Y^{\prime}, \Gamma^{\prime}\right)$ ref. $\bar{k} ; \quad$ (ii) $\tilde{V}=\tilde{\Gamma}(U)$ and $\tilde{V}^{\prime}$ $=\Gamma^{\prime}\left(U^{\prime}\right)$ belong to $\mathfrak{H}$; (iii) $\tilde{V} \in \mathfrak{B}\left(V, \mathfrak{C}_{a}\left(C_{V}\right)\right.$ ) and consequently $V \in \mathfrak{H}_{\alpha}$ by $\left(c^{\prime}\right)$. Set $V^{*}=\Gamma^{*}\left(U^{*}\right)$. Since specializations are compatible with intersectionproduct and algebraic projection, it follows that $\tilde{V} \rightarrow V^{*}$ ref. $\vec{k}$ and $V^{*} \rightarrow \tilde{V}^{\prime}$ ref. $\bar{k}$. Since $\mathfrak{S}$ is an algebraic family, the set of Chow-points of members of $\mathfrak{S}$ is a locally closed subset of a projective space. Hence $V^{*}$ is a member of $\mathfrak{S}$. Since $\Gamma^{*-1}$ is the graph of a non-degenerate projective embedding of $V^{*}$, determined by a divisor in $r \mathscr{C}_{a}\left(C_{V^{*}}\right)$, it follows that $V^{*}$ is not in $\mathfrak{S}_{\alpha}$, otherwise $U^{*}$ would be a member of $\left(\mathfrak{S}_{\alpha}\right)_{r}$. Thus, $V^{*}$ is in $\mathfrak{S}$, in the closure of $\mathfrak{S}_{\alpha}$ but not in $\mathfrak{S}_{\alpha}$. This contradicts to the fact that $\mathfrak{S}$ is an algebraic family. Our corollary is thereby proved.

Proposition 4.3. Let $\mathfrak{F}$ be an irreducible algebraic family of non-singular varieties in a projective space and $r$ a positive integer. Denote by $\mathfrak{F}(r)$ the set of those members $V$ of $\mathfrak{F}$ which carry a divisor $X$ such that $r X \equiv C_{V} \bmod \mathscr{S S}_{a}$. Then $\mathfrak{F}(\boldsymbol{r})$ is an algebraic family. When $\mathfrak{F}$ is defined over a field $k, \mathfrak{F}(\boldsymbol{r})$ is normally algebraic over $k$.

Proof. Let $c_{1}, c_{2}$ be the constants in Theorem 1 relative to a member $V$ of $\mathfrak{F}$, a hyperplane section of $V$ and to our integer $r . g(x)$ in the theorem is now the Hilbert characteristic function of $V$, which is invariant throughout $\widetilde{F}$ by Theorem $A$. $\quad c_{1}$ and $c_{2}$ depend therefore on $\mathfrak{F}$ and $r$ only. Let $q$ be a positive integer such that $q>\max \left(r c_{1}, c_{2}\right)$ and $\mathfrak{u}_{q}$ the set of members $V$ of $\mathfrak{F}$ which carry a positive divisor $Y$ such that $r Y \equiv q C_{V}$ 
$\bmod \mathfrak{S}_{a}$. Suppose that a member $W$ of $\mathfrak{F}$ carries a divisor $X$ such that $r X \equiv C_{W} \bmod \mathbb{S}_{a}$. Then $h^{i}(W, q X)=0$ for $i>0$ and $h^{0}(W, q X)>1$ by our choice of $q$. It follows that $q X \sim Y$ where $Y$ is a positive $W$-divisor. Since $r Y \sim q r X \equiv q C_{W} \bmod \mathfrak{S}_{a}, W$ is a member of $\mathfrak{U}_{q}$ and $\mathfrak{F}(r) \subset \mathfrak{H}_{q}$. Let $q^{\prime}$ be another positive integer similar to $q^{\prime}$. Then $\mathfrak{F}(\boldsymbol{r}) \subset \mathfrak{U}_{q} \cap \mathfrak{U}_{q^{\prime}}$. Take $q$ and $q^{\prime}$ so that they are relatively prime. When $V$ is in $\mathfrak{u}_{q} \cap \mathfrak{u}_{q^{\prime}}$, there is a positive divisor $Y$ (resp. $\left.Y^{\prime}\right)$ on $V$ such that $r Y \equiv q C_{V}\left(\right.$ resp. $\left.r Y^{\prime} \equiv q^{\prime} C_{V}\right) \bmod$ $\mathfrak{S S}_{a}$. When that is so, there is a $V$-divisor $Z$ such that $r Z \equiv C_{V} \bmod \mathfrak{S}_{a}$. It follows that $\mathfrak{F}(r)=\mathfrak{U}_{q} \cap \mathfrak{U}_{q^{\prime}}$ and our proof is reduced to a proof of the fact that the set of Chow-points of members of $\mathfrak{U}_{q}$ is a closed subset of that of $\mathfrak{F}$.

By our choice of $q$, every divisor in $\mathfrak{c}_{a}\left(q C_{V}\right)$ is sufficiently ample for every $V$ in $\mathfrak{F}$ and $h^{0}\left(V, q C_{V}\right)$ does not depend upon $V$ (cf. Proposition 3). Moreover, $\mathfrak{C}_{a}\left(C_{V}\right)^{+}$is a complete total family of divisors on $V$ by Proposition 2. Let us assume that $V$ is a generic member of $\mathfrak{F}$ over $k$ and $V^{\prime}$ a member of $\mathfrak{F}$. Let $G, G^{\prime}$ be respectively be the supports of the Chowvarieties of $\mathfrak{c}_{a}\left(q C_{V}\right)^{+}, \mathfrak{E}_{a}\left(q C_{V^{\prime}}\right)^{+}$. Then $G^{\prime}$ is a unique specialization of $G$ over $k$ over the specialization $V \rightarrow V^{\prime}$ ref. $k$ by Proposition 4. Moreover, when $K$ is a field of definition of $V$ over $k, G$ is the support of a variety defined over $K$ (cf. Theorem $D$ ). Therefore, there is a subvariety $T$ of $F \times \boldsymbol{P}$, where $F$ is the Chow-variety of $\mathfrak{F}$ and $\boldsymbol{P}$ a projective space, such that $v \times \boldsymbol{P} \cap T=v \times G, v^{\prime} \times \boldsymbol{P} \cap T=v^{\prime} \times G^{\prime} \quad$ if we set $\mathfrak{c}(V)=v, \mathfrak{c}\left(V^{\prime}\right)=v^{\prime}$ (cf. [23], Chap. VIII, Theorem 6). Moreover, since $T$ is defined over $k$, the first intersection is proper on $F \times \boldsymbol{P}$. Let $A$ be the projection of $T$ on $\boldsymbol{P}$. The set of points of $A$ which represents cycles of the form $r Z$ in the ambient projective space of $F$ forms a closed subset $A_{0}$ of $A$. Then the set $T_{0}$ of points on $T$ which projects into $A_{0}$ is a closed subset of $T$. Clearly, a member $V^{\prime}$ of $\mathfrak{F}$ is in $\mathfrak{H}_{q}$ if and only if there is a point $\left(v^{\prime}, a^{\prime}\right)$ in $T_{0}$ such that $\mathfrak{c}\left(V^{\prime}\right)=v^{\prime}$. Denote by $F_{0}$ the projection of $T_{0}$ on $F$ and by $U_{q}$ the set of Chow-points of members $\mathfrak{u}_{q} . U_{q}$ is contained in $F_{0}$ and $F_{0}$ is the closure of $U_{q}$ on $F$. But the relation $r Y^{\prime} \equiv q C_{V}$, $\bmod \mathscr{S}_{a}$ is preserved by specialization on $F$. Thus $F_{0}=U_{q}$ and our proposition follows from this easily.

§ 5. On locally closed subsets. Let $U$ be an abstract variety. As is well-known, a locally closed subset of $U$ in the sense of Zariski topology is given in the form $F^{\prime}=F-F \cap X$, where $F$ and $X$ are closed subsets 
of $U$. Let the $F_{\alpha}$ be the components of $F$ and set $F_{\alpha}^{\prime}=F_{\alpha}-F_{\alpha} \cap X$. Then $F^{\prime}$ is the union of the $F_{\alpha}^{\prime}$. The $F_{\alpha}^{\prime}$ are called the components of $F^{\prime}$. As usual, we take on $F^{\prime}$ and on the $F_{\alpha}^{\prime}$ induced topology.

Proposition 5.1. Let $U$ be an abstract variety, $F$ a locally closed subset of $U$ and the $F_{\alpha}$ the components of $F$. Let $k$ be a common field of definition for $U$ and for the $F_{\alpha}$. Then (a) when $Z$ is a subvariety of $U$, defined over $k$, such that a generic point $z$ of $Z$ over $k$ is contained in $F_{\alpha}$, the restriction $Z^{\prime}=Z \cap F$ of $Z$ in $F$ is contained in $F_{\alpha}$. Moreover, (b) when $Y$ is a subset of $F, Y$ is a closed subset of $F$ if and only if $Y_{\alpha}=Y \cap F_{\alpha}$ is closed on $F_{\alpha}$ for all $\alpha$.

Proof. When $\bar{F}$ is the closure of $F$ on $U$, there is a closed subset $X$ of $U$ such that $F=\bar{F}-X . \quad U-X$ is an abstract variety. Therefore, it is enough to prove our proposition when $F$ is a closed subset of $U$.

(a) follows easily from the definitions. In order to prove (b), first assume that $Y$ is closed on $F$. Then $Y$ is a finite union of subvarieties $Z$ of $U$ which are contained in $F$. Hence $Y$ is closed on $U$ and the $Y_{\alpha}=$ $Y \cap F_{\alpha}$ are closed on the $F_{\alpha}$. Conversely, assume that the $Y_{\alpha}$ are closed on the $F_{\alpha} . Y_{\alpha}$ is a finite union of subvarieties $Z_{\alpha, i}$ of $U$, contained in $F_{\alpha}$. Since $Y$ is the union of the $Z_{\alpha, i}$, it is closed on $U$ and hence on $F$.

\section{REFERENCES}

[1] W.L. Chow, "On the connectedness theorem in algebraic geometry", American Journal of Mathematics, vol. 81, (1959), pp. 1033-1074.

[2] - "Algebraic systems of positive cycles in an algebraic variety", American Journal of Mathematics, vol. 72, (1950), pp. 247-283.

[ 3 ] W.L. Chow-v.d. Waerden, "Zur algebraischen Geometrie IX", Mathematische Annalen, vol. 113, (1937), pp. 692-704.

[4] A. Grothendieck, Éléments de géometrie algébriques, I-IV, Publications Mathematiques Institut des Hautes Études Scientifiques, Paris.

[5] - "Les schémas de Hilbert", Seminaire Bourbaki, $n^{0} 221$, (1960/61).

[6] , "Les schémas de Picard: Propriétés générales", Séminaire Bourbaki, $n^{0}$ 236, (1961/62).

[ 7 ] H. Hironaka, "A note on algebraic geometry over ground rings," Illinois Journal of Mathematics, vol. 2 (1958), pp. 355-366.

[8 ] W.V.D. Hodge, The theory and applications of harmonic integrals, Cambridge University Press (1959).

[8'] S. Kleiman, "Toward a numerical theory of ampleness," Thesis at Harvard University (1965).

[ 9 ] K. Kodaira and D.C. Spencer, "On deformations of complex analytic Structures, I, II," Annals of Mathematics, vol. 67 (1958), pp. 328-466.

[10] S. Lang, Abelian varieties, Interscience Tracts, No. 7, 1959. 
[11] T. Matsusaka, "On the algebraic construction of the Picard variety, II," Japanese Journal of Mathematics, vol. 22 (1952), pp. 51-62.

[12] - "On the algebraic families of positive divisors," Journal of the Mathematical Society of Japan, vol. 5 (1953), pp. 115-136.

[13] - "The criteria for algebraic equivalence and the torsion group," American Journal of Mathematics, vol. 79 (1957), pp. 53-66.

[14] - "Polarized varieties, fields of moduli, etc.," American Journal of Mathematics, vol. 80 (1958), pp. 45-82.

[15] - Theory of Q-varieties, Publications of the Mathematical Society of Japan, No. 8, 1965.

[16] T. Matsusaka and D. Mumford, "Two fundamental theorems on deformations of polarized varieties," American Journal of Mathematics, vol. 86 (1964), pp. 668-684.

[17] D. Mumford, Geometric invariant theory, Berlin-Heidelberg-New York: Springer, 1965.

[18] Y. Nakai, "Note on the intersection of an algebraic variety with the generic hyperplane," Memoirs of the College of Science, Kyoto University ,vol. 26 (1951), pp. 185-187.

[19] S. Lang and A. Néron, "Rational points of Abelian varieties over function fields," American Journal of Mathematics, vol. 81 (1959), pp. 95-118.

[20] J.-p. Serre, "Faisceaux algébriques cohérents," Annals of Mathematics, vol. 61 (1955), pp. $197-278$.

[21] G. Shimura, "Reduction of algebraic varieties with respect to a discrete valuation of the basis field," American Journal of Mathematics, vol. 77 (1955), pp. 134-176.

[22] — "Modules des variétés abéliennes polarisées et fonctions modulaires," Séminaire H. Cartan, E.N.S. (1957/58).

[22'] E. Snapper, "Multiples of divisors," Journal of Mathematics and Mechanics, vol. 8 (1959), pp. 967-992.

[23] A. Weil, Foundations of algebraic geometry, American Mathematical Society Colloquium Publications, No. 29 (1960).

[24] _ "On algebraic groups of transformations," American Journal of Mathematics, Vol. 77 (1955), pp. 355-391.

[25] — - "On the theory of complex multiplications, "Proceedings, International Symposium on Algebraic Number Theory, Nikko, Japan, 1955.

[26] O. Zariski, "Scientific report on the second summer institute, III," Bulletin of the American Mathematical Society, Vol. 62 (1956), pp. 117-141.

[27] _ _ "Complete linear systems on normal varieties and a generalization of a lemma of Enriques-Severi," Annals of Mathematics, Vol. 55 (1952), pp. 552-592.

Brandeis University 